\title{
Geometric information flows and G. Perelman entropy for relativistic classical and quantum mechanical systems
}

\author{
Sergiu I. Vacaru ${ }^{1,2,3, a}$ \\ ${ }^{1}$ Physics Department, California State University, Fresno, Fresno, CA 93740, USA \\ ${ }^{2}$ Project IDEI, University "Al. I. Cuza” Iaşi, Iaşi, Romania \\ ${ }^{3}$ Present address: Department of Theoretical Physics and Computer Modelling, Yuriy Fedkovych Chernivtsi National University, \\ 101 Storozhynetska street, Chernivtsi 58029, Ukraine
}

Received: 24 May 2019 / Accepted: 24 June 2020 / Published online: 18 July 2020

(C) The Author(s) 2020

\begin{abstract}
This work consists an introduction to the classical and quantum information theory of geometric flows of (relativistic) Lagrange-Hamilton mechanical systems. Basic geometric and physical properties of the canonical nonholonomic deformations of G. Perelman entropy functionals and geometric flows evolution equations of classical mechanical systems are described. There are studied projections of such F- and W-functionals on Lorentz spacetime manifolds and three-dimensional spacelike hypersurfaces. These functionals are used for elaborating relativistic thermodynamic models for Lagrange-Hamilton geometric evolution and respective generalized Hamilton geometric flow and nonholonomic Ricci flow equations. The concept of nonholonomic W-entropy is developed as a complementary one for the classical Shannon entropy and the quantum von Neumann entropy. There are considered geometric flow generalizations of the approaches based on classical and quantum relative entropy, conditional entropy, mutual information, and related thermodynamic models. Such basic ingredients and topics of quantum geometric flow information theory are elaborated using the formalism of density matrices and measurements with quantum channels for the evolution of quantum mechanical systems.
\end{abstract}

\section{Contents}

1 Introduction . . . . . . . . . . . 2

2 A Hessian type geometrization of Lagrange-Hamilton mechanics ............... 3

Address for post correspondence in 2019-2020 as a visiting senior researcher at YF CNU Ukraine is: 37 Yu. Gagarin street, ap. 3, Chernivtsi, Ukraine, 58008; the UAIC affiliation is preserved for a former hosted project IDEI during 2012-2015.

a e-mail: sergiu.vacaru@gmail.com; sergiuvacaru@mail.fresnostate.edu (corresponding author)
2.1 Canonic nonholonomic models of LagrangeHamilton geometry . . . . . . . . . 3

2.1.1 Phase spacetimes with Lagrange-Hamilton generating functions and Hessian metrics

2.1.2 Nonlinear connections, adapted frames, and distinguished metrics . . . . . . 5 5

2.1.3 Hamilton-Jacoby, Euler-Lagrange, and semi-spray equations and $\mathrm{N}$-connections . 5

2.1.4 Canonical d-metric and almost complex structures ............ 6

2.2 Linear connections and curvatures for LagrangeHamilton spaces . . . . . . . . . . . . 7

2.2.1 Distinguished connections, $\mathrm{N}$-adapted distortions and curvatures . . . . . . 7

2.2.2 The Ricci and Einstein d-tensors on phase spaces and (co) vector bundles . . . . . 8

2.2.3 Physically important d-connections for geometric mechanics ......... 8

3 Geometric flow evolution of classical mechanical systems 9

3.1 Relativistic geometric flows and Perelman's thermodynamics for phase spacetimes . . . . 10

3.1.1 Perelman-Lagrange and Perelman-Hamilton functionals . . . . . . . . 10

3.1.2 Relativistic thermodynamic models for Lagrange-Hamilton geometric evolution . 11

3.1.3 Curved spaces emerging from relativistic phase space geometric evolution . . . . 13

3.1.4 Effective nonholonomic 3-d space like hypersurface $\mathrm{F}$ - and W-functionals . . . 13

3.2 Generalized R. Hamilton flow evolution equations and geometric mechanics . . . . . . . 14

3.2.1 Riemannian geometric flows on 3-d spacelike hypersurface . . . . . . . . . . 14

3.2.2 Geometric flow equations for relativistic Lagrange-Hamilton systems . . . . . . 14 
3.2.3 Nonholonomic Ricci solitons, emergent gravity, and geometric mechanics . . . . 15

4 Classical and quantum mechanical geometric information flow theories . . . . . . . . . . . 15

4.1 Geometric information flow theory of classical mechanical systems . . . . . . . . . 15

4.1.1 Shannon entropy and geometric flow entropy in information theories . . . . . . 15

4.1.2 Conditional entropy and geometric information flows GIF . . . . . . . . . . . 16

4.1.3 Relative GIF entropy and monotonicity . 18

4.2 Basic ingredients of the quantum geometric information flow theory . . . . . . . . . . . 19

4.2.1 Density matrices and properties of quantum entropies for GIFs . . . . . . . . . 19

4.2.2 Properties of entropies for QGIFs . . . . 21

4.2.3 Measurements for QGIFs and quantum channels ............. 23

5 Outlook and conclusions . . . . . . . . . . 25

References ................ 25

\section{Introduction}

One of the most remarkable success in modern mathematics is the proof of the Poincaré-Thurston conjecture due to $\mathrm{G}$. Perelman [1-3]. We cite here most important related works on W. Thurston's classification of three dimensional, 3-d, manifolds, [4-6]; then D. Friedman's geometric flow evolution equations derived for renorm-group considerations in quantum field theory and condensed matter physics, see [79]; and R. Hamilton [10-12] fundamental contributions to Ricci flow theory. The monographs [13-15] can be considered for rigorous proofs and reviews of results in geometric analysis and topology. ${ }^{1}$ A series of our works were elaborated in a 'geometry and physics' style involving generalizations for relativistic systems and applications in modern physics and cosmology. We cite [19-22], for geometric flows of Lagrange-Finsler spaces and nonholonomic manifolds and algebroids; [23], on noncommutative geometric flow evolution theories; [24,25], for respective super-Ricci flows and thermodynamics of relativistic Ricci flows; and a series of

\footnotetext{
$\overline{1}$ We emphasize that the terms Hamilton mechanics and Hamilton equations for Ricci flows are related to the names of two different famous scientists. In the first case, it refers to William R. Hamilton who formulated in 1834 his Hamiltonian mechanics starting from Lagrangian mechanics (a previous reformulation for classical mechanics introduced by Joseph Louis Lagrange in 1788). On mathematical and physical approaches and historical remarks on Lagrange and Hamilton mechanics, see [1618]. In the second case, Richard Hamilton is known because of his achievements on the Ricci flows theory and applications in topology and geometric analysis [10-12].
}

works [26-29] related to modified gravity theories, MGTs, and cosmology, see reviews [30-35].

Above mentioned directions for advanced studies in geometry and mathematical physics were developed using G. Perelman's concepts of F- and W-entropy Perelman. Such values were constructed as A. M. Lyapunov type functionals [36] which for geometric flows of Riemannian metrics are determined by Ricci tensors and scalars. We defined their nonholonomic deformations (equivalently, anholonomic, i.e. subjected to non-integrable constraints) for various generalized geometric and physical models. The W-entropy is like a "minus entropy" and it describes some nonholonomic entropic flows of various classical and quantum physical systems. The concept of W-entropy is different from the Shannon, von Neumann, or other type, entropy used in modern thermodynamics and classical/ quantum information theory, see $[37,38]$ and references therein. With respect to various developments and applications in modern gravity and black hole, BH, and cosmology information theory (based on areaentropy, holography and similar concepts), the constructions with the G. Perelman entropy and modifications seem to be more general than those based on the Bekenstein-Hawking thermodynamics [39-42]. On recent research with "nonarea and non-holographic" entropies for geometric flows and gravity, see details and discussions in [24-29].

This paper is the 4 th partner one in a series of previous works [27-29]. The goal is to elaborate on certain most important principles and methods for formulating classical and quantum information theories encoding geometric flows of relativistic Lagrange-Hamilton mechanical systems. We shall also consider spacetime configurations emerging as nonholonomic Ricci solitons, and their analogous geometric thermodynamic models. This new approach to formulating geometric information flow, GIF, theories is based on the concept of G. Perelman entropy and a geometrization of physical theories due to J. Kern [43] and M. Matsumoto [44,45]. The Kern-Matsumoto ideas were that classical mechanics can be formulated as Finsler like geometries without homogeneity conditions on respective Lagrange and/or Hamilton generating functions on (co) vector and tangent bundles, see a modern axiomatic approach and historical remarks in [34,35]. For such a geometric formulation, the classical and quantum field and flow evolution theories can be characterized by certain generalized Perelman's entropy like functionals. These functionals allow new developments and applications to classical and quantum information theories. In this work, there are not studied emergent (modified) gravity theories even we provide certain generalized classical and quantum mechanical entropic functionals from which generalized Einstein equations can be derived. We cite [26,27,29] for recent results on exact solutions and modified Ricci flow theories and gravity. 
It is assumed that the reader has a background knowledge about mathematical physics and geometric methods in QFT and (modified) gravity theories, and certain familiarity with fiber bundles and (non) linear connections, nonholonomic mechanics and geometric thermodynamics, see [23, 25,46] and references therein. Certain other sources of literature on classical and quantum information theory and modern physics [37,38,47-49] are listed for more comprehensive treatments of the subjects that we touch in our developments.

This work is organized as follows: In Sect. 2, we summarize necessary results on J. Kern's approach to geometrization of classical Lagrange and Hamilton mechanics. There are also defined the geometric objects which are important for relativistic generalizations of such geometric models on (co) tangent Lorentz bundles. Section 3 is devoted to the theory of nonholonomic geometric flow evolution of classical relativistic mechanical systems. There are introduced the Perelman-Lagrange and Perelman-Hamilton functionals for geometric mechanics flows on curved phase spacetimes and their reductions on 4-d Lorentz manifolds (as certain emergent flow evolution gravity theories) and 3-d space like hypersurfaces. Corresponding relativistic thermodynamics values are defined. Generalized R. Hamilton geometric flow evolution of flow equations are derived for relativistic LagrangeHamilton systems. Self-similar configurations are defined as nonholonomic Ricci-Lagrange and Ricci-Hamilton solitons and studied the conditions certain analogous mechanical models define emergent vacuum gravitational configurations. A brief introduction to theories of classical and quantum mechanical geometric information flow, GIF, is provided in Sect. 4. We define and study basic properties of GIF entropies and basic ingredients of the quantum geometric flow information, QGIF, theory and respective thermodynamics for quantum channels. Finally, we draw conclusions and speculate on further perspectives in Sect. 5 .

\section{A Hessian type geometrization of Lagrange-Hamilton mechanics}

We develop an approach to geometrization of relativistic Lagrange and Hamilton mechanics on tangent and cotangent Lorentz manifolds (respectively, $T V$ and $T^{*} V$ ) on a Lorentz manifold $V$ of dimension $\operatorname{dim} V=4$ and with local Euclidean signature $(+++-)$, see $[34,35]$ for details and historical remarks. The concept of Lagrange space was proposed in [43] as an alternative geometrization for nonrelativistic mechanics outlined in [16-18]. The main idea in such Hessian geometric models (with a so-called vertical, or covertical, metric determined by a Lagrange, or Hamilton, generating function) is to drop the homogeneity condition for generating functions and apply Finselr and almost Kähler geometry methods to classical field theories and mechanics
$[44,45]$. Here we note that other approaches on geometrization of classical mechanics and fields, for instance, the polysimplectic formalism (see [18], references therein and further developments in modern literature), do not allow an unified formulation of models for geometric flow evolution, thermodynamics and statistics, (modified) gravity theories and classical and quantum information. In our works [1926,26,27,27,28,28,29,29], using constructions with generalized Finsler like Hessian geometrization of LagrangeHamilton systems in mathematical relativity, cosmology and particle physics, various directions were developed for classical and quantum (non) commutative / supersymetric field theories, in modified gravity, inhomogeneous cosmology and theory of nonholonomic geometric flows.

\subsection{Canonic nonholonomic models of Lagrange-Hamilton geometry}

Geometrization of classical nonrelativistic and relativistic mechanical systems can be performed on a Riemannian or Lorentz manifold $V$ and it tangent $T V$ and cotangent $T^{*} V$ bundles enabled with (pseudo) Riemannian metrics with local (pseudo) Euclidean signature.

\subsubsection{Phase spacetimes with Lagrange-Hamilton generating functions and Hessian metrics}

We call $T V$ and/or $T^{*} V$ as phase spaces or phase spacetimes depending on signatures of metrics they are enabled. In a typical case, there are considered corresponding quadratic line elements determined by total phase space metrics with signature $(+++-;+++-)$,

$$
\begin{aligned}
d s^{2}= & g_{\alpha \beta}\left(x^{k}\right) d u^{\alpha} d u^{\beta}=g_{i j}\left(x^{k}\right) d x^{i} d x^{j} \\
& +\eta_{a b} d y^{a} d y^{b}, \text { for } y^{a} \sim d x^{a} / d \tau ; \text { and } / \text { or } \\
d^{\prime} s^{2}= & g_{\alpha \beta}\left(x^{k}\right) d^{\prime} u^{\alpha} d^{\prime} u^{\beta}=g_{i j}\left(x^{k}\right) d x^{i} d x^{j} \\
& +\eta^{a b} d p_{a} d p_{b}, \text { for } p_{a} \sim d x_{a} / d \tau .
\end{aligned}
$$

In these formulas, the local frame and dual frame (co-frame) coordinates are labeled respectively. We write $u^{\alpha}=\left(x^{i}, y^{a}\right)$, (or in brief, $u=(x, y)$ ), on the tangent bundle $T V$; and ${ }^{\prime} u^{\alpha}=\left(x^{i}, p_{a}\right)$, (or in brief, ' $\left.u=(x, p)\right)$, on the cotangent bundle $T^{*} V$. The total phase space metrics $g_{\alpha \beta}(u)$ and ' $g_{\alpha \beta}$ ( ' $\left.u\right)$ are determined, for such examples, by a pseudoRiemannian spacetime metric $g=\left\{g_{i j}(x)\right\}$ with the LeviCivita connection, LC-connection, $\nabla$, which is metric compatible and with zero torsion. In diagonal form, the vertical metric $\eta_{a b}$ and its dual $\eta^{a b}$ are standard Minkowski metrics, $\eta_{a b}=\operatorname{diag}[1,1,1,-1]$ used for computations in typical fibers of respective (co) tangent bundles. The mechanical models can be elaborated for general frame/ coordinate transforms in total spaces when the metric structures can be parameterized equivalently by the same h-components of 
$g_{\alpha \beta}\left(x^{k}\right)$ and ' $g_{\alpha \beta}\left(x^{k}\right)=g_{\alpha \beta}\left(x^{k}\right)$, but different (co) fiber metrics $g_{a b}(x, y)$ and $g^{a b}(x, p)$ than those considered in (1) and (2). ${ }^{2}$

A relativistic 4-d model of Lagrange space $L^{3,1}=$ $(T V, L(x, y))$ is determined by a fundamental function (equivalently, generating function) $T V \ni(x, y) \rightarrow L(x, y)$ $\in \mathbb{R}$, i.e. a real valued function (in brief, called a Lagrangian or a Lagrange density) which is differentiable on $\widetilde{T V}:=$ $T V /\{0\}$, for $\{0\}$ being the null section of $T V$, and continuous on the null section of $\pi: T V \rightarrow V$. Such a relativistic model is regular if the Hessian metric (equivalently, v-metric)

$\widetilde{g}_{a b}(x, y):=\frac{1}{2} \frac{\partial^{2} L}{\partial y^{a} \partial y^{b}}$

is non-degenerate, i.e. det $\left|\widetilde{g}_{a b}\right| \neq 0$, and of constant signature.

In modern literature on geometric mechanics, kinetics and statistical mechanics of locally anisotropic processes (see a review of such results and references in $[34,35]$ ), there are used constructions on cotangent bundles with such a concept: A 4-d relativistic model of Hamilton space $H^{3,1}=$ $\left(T^{*} V, H(x, p)\right)$ is constructed for a fundamental function (equivalently, generating Hamilton function, in brief, Hamiltonian or Hamilton density) on a Lorentz manifold $V$. One considers that $T^{*} V \ni(x, p) \rightarrow H(x, p) \in \mathbb{R}$ defines a real valued function being differentiable on $\widetilde{T^{*} V}:=T^{*} V /\left\{0^{*}\right\}$, for $\left\{0^{*}\right\}$ being the null section of $T^{*} V$, and continuous on the null section of $\pi^{*}: T^{*} V \rightarrow V$. Such a relativistic mechanical model is regular if the Hessian (cv-metric)

$$
{ }^{\prime} \widetilde{g}^{a b}(x, p):=\frac{1}{2} \frac{\partial^{2} H}{\partial p_{a} \partial p_{b}}
$$

is non-degenerate, i.e. $\operatorname{det}\left|{ }^{\prime} \widetilde{g}^{a b}\right| \neq 0$, and of constant signature.

For Lagrange and Hamilton spaces, we can consider Legendre transforms $L \rightarrow H(x, p):=p_{a} y^{a}-L(x, y)$ and $y^{a}$ determining solutions of the equations $p_{a}=\partial L(x, y) / \partial y^{a}$. In a similar manner, the inverse Legendre transforms can be introduced, $H \rightarrow L$, when

$L(x, y):=p_{a} y^{a}-H(x, p)$

for $p_{a}$ determining solutions of the equations $y^{a}=$ $\partial H(x, p) / \partial p_{a}$.

\footnotetext{
$\overline{2}$ There are used such conventions for indices: the "horizontal" indices, h-indices, run values $i, j, k, \ldots=1,2,3,4$; the vertical indices, vvertical, run values $a, b, c \ldots=5,6,7,8$; respectively, the v-indices can be identified/ contracted with h-indices 1, 2, 3, 4 for lifts on total (co) tangent Lorentz bundles, when $\alpha=(i, a), \beta=(j, b), \gamma=(k, c), \ldots=$ $1,2,3, \ldots 8$. We shall consider letters labelled by an abstract left up/low symbol "'" (for instance, ' $u^{\alpha}$ and ' $g_{\alpha \beta}$ ) in order to emphasize that certain geometric/physical objects are defined on $T^{*} V$. In similar forms, we can consider indices for lower and higher dimensions than $4+4$, or other type signatures.
}

The non-Riemannian total phase space geometries are characterized by nonlinear quadratic line elements

$d s_{L}^{2}=L(x, y)$, for models on $T V ; \quad d^{\prime}$

$s_{H}^{2}=H(x, p)$, for models on $T^{*} V$.

We can elaborate on geometric and physical theories with an effective phase spacetime modelled on (co) tangent Lorentz bundles endowed with generalized frame, metric and linear and nonlinear connection structures determined by nonlinear quadratic line elements and (6). For certain special cases, such values transform correspondingly into quadratic line elements (1) and (2).

The Hessians $\widetilde{g}_{a b}$ and ' $\widetilde{g}^{a b}$ are labeled by a tilde " $\sim$ " in order to emphasize that such conventional v- and cv-metrics are defined canonically by respective Lagrange and Hamilton generating functions. For simplicity, we can work with such regular metrics even, in principle, mechanical models with degenerate Hessians are also studied in modern mechanics and field theories. Considering general frame/ coordinate transforms on phase spaces, we can express any "tilde" Hessian in a general quadratic form, respectively as a vertical metric (v-metric), $g_{a b}(x, y)$, and/or co-vertical metric (cvmetric), ' $g^{a b}(x, p)$. Inversely, if a v-metric (cv-metric) is prescribed, we can introduce respective (co) frame /coordinate systems, when such values can transformed into certain canonical ones, with "tilde" values. In general, a v-metric $g_{a b}$ is different from the inverse of a cv-metric ' $g^{a b}$, i.e. from the ' $g_{a b}$. Nevertheless, certain relations between such values can be found via Legendre transforms. We shall omit tildes on geometrical/ physical objects on respective phase spaces if certain formulas hold in general (not only canonical) forms and/or that will not result in ambiguities.

For simplicity, the bulk of geometric constructions in this paper will be performed for (effective and/or generalized) Hamilton spaces if that will not result in ambiguities. We shall consider that via corresponding frame and Legendre transforms, or homogeneity conditions, we can generate necessary type Lagrange/ Finsler/ Cartan configurations. ${ }^{3}$

\footnotetext{
${ }^{3}$ A relativistic 4-d model of Finsler space is an example of Lagrange space when a regular $L=F^{2}$ is defined by a fundamental (generating) Finsler function subjected to certain additional conditions: 1) $F$ is a real positive valued function which is differential on $\widetilde{T V}$ and continuous on the null section of the projection $\pi: T V \rightarrow V ; 2)$ it is satisfied the homogeneity condition $F(x, \lambda y)=|\lambda| F(x, y)$, for a nonzero real value $\lambda$; and 3) the Hessian (3) is defined by $F^{2}$ in such a form that in any point $\left(x_{(0)}, y_{(0)}\right)$ the $\mathrm{v}$-metric is of signature $(+++-)$. In a similar form, we can define relativistic Cartan spaces $C^{3,1}=(V, C(x, p))$, when $H=C^{2}(x, p)$ is 1 -homogeneous on co-fiber coordinates $p_{a}$.
} 


\subsubsection{Nonlinear connections, adapted frames, and distinguished metrics}

A complete geometrization of mechanical models is not possible if we use only Lagrange-Hamilton functions and respective (non) linear quadratic elements. There are necessary additional concepts and definition of new geometric objects like the nonlinear connection structure, the distinguished linear connection, various distinguished geometric objects etc., see details and motivations in [34,35].

A nonlinear connection, N-connection, structure for $T V$, or $T^{*} V$, is defined as a Whitney sum of conventional $h$ and $v$-distributions, or $h$ and $c v$-distributions,

$$
\begin{aligned}
\mathbf{N}: T T V & =h T V \oplus v T V, \text { or } \quad{ }^{\prime} \mathbf{N}: T T^{*} V \\
& =h T^{*} V \oplus v T^{*} V .
\end{aligned}
$$

Parameterizing locally the $\mathrm{N}$-connections with respect to coordinate bases by corresponding coefficients $\mathbf{N}=\left\{N_{i}^{a}\right\}$ and $\mathbf{N}=\left\{{ }^{\prime} N_{i a}\right\}$, we obtain by explicit constructions that decompositions/splitting (7) define respective systems of $\mathrm{N}$ linear (i.e. $\mathrm{N}$-adapted) bases

$$
\begin{aligned}
\mathbf{e}_{\alpha}= & \left(\mathbf{e}_{i}=\frac{\partial}{\partial x^{i}}-N_{i}^{a}(x, y) \frac{\partial}{\partial y^{a}}, e_{b}=\frac{\partial}{\partial y^{b}}\right), \\
\mathbf{e}^{\alpha}= & \left(e^{i}=d x^{i}, \mathbf{e}^{a}=d y^{a}\right. \\
& \left.+N_{i}^{a}(x, y) d x^{i}\right), \text { and } / \text { or } \\
{ }^{\prime} \mathbf{e}_{\alpha}= & \left({ }^{\prime} \mathbf{e}_{i}=\frac{\partial}{\partial x^{i}}-{ }^{\prime} N_{i a}(x, p) \frac{\partial}{\partial p_{a}},{ }^{\prime} e^{b}=\frac{\partial}{\partial p_{b}}\right), \\
{ }^{\prime} \mathbf{e}^{\alpha}= & \left({ }^{\prime} e^{i}=d x^{i},{ }^{\prime} \mathbf{e}_{a}=d p_{a}+{ }^{\prime} N_{i a}(x, p) d x^{i}\right) .
\end{aligned}
$$

The N-connection coefficients and necessary types of (co) frame/ coordinate transforms can be used for constructing lifts of metric structures $(V, g)$ to respective nonholonomic (co)tangent bundles, ( $\mathbf{T V}, \mathbf{N}, \mathbf{g})$ and $\left(\mathbf{T}^{*} \mathbf{V},{ }^{\prime} \mathbf{N},{ }^{\prime} \mathbf{g}\right){ }^{4}$

We can consider various type of metric structures on a tangent, $\mathbf{T V}$, and/or cotangent, $\mathbf{T}^{*} \mathbf{V}$, Lorentz bundles. This can be used for elaborating mechanical models, thermodynamic and kinetic theories and generalizations of the Einstein gravity. Such metric structures can be parameterized by frame transforms in $\mathrm{N}$-adapted form, i.e. as distinguished metrics (d-metrics)

$$
\begin{aligned}
\mathbf{g}= & \mathbf{g}_{\alpha \beta}(x, y) \mathbf{e}^{\alpha} \otimes \mathbf{e}^{\beta}=g_{i j}(x) e^{i} \otimes e^{j} \\
& +\mathbf{g}_{a b}(x, y) \mathbf{e}^{a} \otimes \mathbf{e}^{a} \text { and } / \text { or } \\
' \mathbf{g}= & { }^{\prime} \mathbf{g}_{\alpha \beta}(x, p){ }^{\prime} \mathbf{e}^{\alpha} \otimes{ }^{\prime} \mathbf{e}^{\beta}=g_{i j}(x) e^{i} \otimes e^{j} \\
& +{ }^{\prime} \mathbf{g}^{a b}(x, p){ }^{\prime} \mathbf{e}_{a} \otimes{ }^{\prime} \mathbf{e}_{b} .
\end{aligned}
$$

\footnotetext{
4 Boldface symbols are used in order to emphasize that certain geometric/physical objects are considered in $\mathrm{N}$-adapted form for certain phase spaces and/or spacetime enabled with $\mathrm{N}$-connection structure and when the coefficients of tensors, spinors, and fundamental geometric objects can be computed with respect to $\mathrm{N}$-elongated bases of type (8).
}

In this work, such metrics on conventional 8-d manifolds are of signature $(+,+,+,-,+,+,+,-)$ but for elaborating non-relativistic mechanical/ thermodynamical / statistical models other type signatures can be considered. For instance, a pseudo-Riemannian metric $g_{i j}(x)$ can be subjected to the condition that it defines a solution of the standard Einstein equations in GR, or a MGT, with a corresponding base Lorentz manifold V. For various mechanical and thermodynamical models, there are necessary additional geometrically and physically motivated assumptions on how nonlinear quadratic elements of type or (6), and/or (9), or (10), encode local anisotropies, inhomogeneous structures, modified dispersion relations etc.

\subsubsection{Hamilton-Jacoby, Euler-Lagrange, and semi-spray equations and $N$-connections}

Let us consider that a spacetime Lorentzian (or a space Riemannian) manifold $\mathbf{V}$ is endowed with a metric $h g=$ $\left\{g_{i j}(x)\right\}$ of signature $(3,1)$ (or of Euclidean signature). Using frame/generalized coordinate transforms on base and total spaces, metrics can be deformed to off-diagonal metrics depending on velocity/ momentum coordinates, including horizontal components of Hessian type.

Considering a regular curve $c(\tau)$ defined $c: \tau \in[0,1] \rightarrow$ $x^{i}(\tau) \subset U \subset V$, for a real parameter $\tau$, we can construct a lifted to $\pi^{-1}(U) \subset \widetilde{T V}$ defining a curve in the total space, when $\widetilde{c}(\tau): \tau \in[0,1] \rightarrow\left(x^{i}(\tau), y^{i}(\tau)=d x^{i} / d \tau\right)$ with a non-vanishing v-vector field $d x^{i} / d \tau$. Using a canonical symplectic structure $\theta:=d p_{i} \wedge d x^{i}$ on $T^{*} V$ and a unique vector filed $\widetilde{X}_{H}:=\frac{\partial \widetilde{H}}{\partial p_{i}} \frac{\partial}{\partial x^{i}}-\frac{\partial \widetilde{H}}{\partial x^{i}} \frac{\partial}{\partial p_{i}}$ defined by $\widetilde{H}$, we construct an equation $i_{\widetilde{X}_{H}} \theta=-d \widetilde{H}$. We write $\wedge$ for the antisymmetric product where $i_{\widetilde{X}_{H}}$ denotes the interior produce defined by $\widetilde{X}_{H}$. This allows us to formulate and prove using an explicit calculus for any functions ${ }^{1} f(x, p)$ and ${ }^{2} f(x, p)$ on $T^{*} V$ and a canonical Poisson structure $\left\{{ }^{1} f,{ }^{2} f\right\}:=\theta\left(\widetilde{X}_{1}, \widetilde{X}_{2}\right)$.

The canonical Hamilton-Jacobi equations are defined using above canonical Poisson structure,

$\frac{d x^{i}}{d \tau}=\left\{\widetilde{H}, x^{i}\right\} \quad$ and $\quad \frac{d p_{a}}{d \tau}=\left\{\widetilde{H}, p_{a}\right\}$.

The dynamics of a probing point particle in $L$-dual effective phase spaces $\widetilde{H}^{3,1}$ and $\widetilde{L}^{3,1}$ is described equivalently by the Hamilton equations $\frac{d x^{i}}{d \tau}=\frac{\partial \widetilde{H}}{\partial p_{i}}$ and $\frac{d p_{i}}{d \tau}=-\frac{\partial \widetilde{H}}{\partial x^{i}}$, or as EulerLagrange equations, $\frac{d}{d \tau} \frac{\partial \widetilde{L}}{\partial y^{i}}-\frac{\partial \widetilde{L}}{\partial x^{i}}=0$. In their turn, these equations are equivalent to the nonlinear geodesic (semispray) equations

$\frac{d^{2} x^{i}}{d \tau^{2}}+2 \widetilde{G}^{i}(x, y)=0$,

for $\widetilde{G}^{i}=\frac{1}{2} \widetilde{g}^{i j}\left(\frac{\partial^{2} \widetilde{L}}{\partial y^{i}} y^{k}-\frac{\partial \widetilde{L}}{\partial x^{i}}\right)$, with $\widetilde{g}^{i j}$ being inverse to $\widetilde{g}_{i j}$ (3). 
The equations (11) show that point like probing particles move not along usual geodesics as on Lorentz manifolds but follow some nonlinear geodesic equations determined by generating Lagrange functions and their Hessians.

Using the constructions from above section, we prove there are canonical $\mathrm{N}$-connections determined by generating Lagrange/ Hamilton functions following formulas

$$
\begin{aligned}
& \text { ' } \widetilde{\mathbf{N}}=\left\{{ }^{\prime} \widetilde{N}_{i j}:=\frac{1}{2}\left[\left\{{ }^{\prime} \widetilde{g}_{i j}, \widetilde{H}\right\}-\frac{\partial^{2} \widetilde{H}}{\partial p_{k} \partial x^{i}} \cdot \widetilde{g}_{j k}-\frac{\partial^{2} \widetilde{H}}{\partial p_{k} \partial x^{j}} ' \widetilde{g}_{i k}\right]\right\} \\
& \text { and } \widetilde{\mathbf{N}}=\left\{\widetilde{N}_{i}^{a}:=\frac{\partial \widetilde{G}}{\partial y^{i}}\right\},
\end{aligned}
$$

where ${ }^{\prime} \widetilde{g}_{i j}$ is inverse to ${ }^{\prime} \widetilde{g}^{a b}(4)$. Introducing these canonical $\mathrm{N}$-connection coefficients into formulas (8), we prove that there are canonical $\mathrm{N}$-adapted (co) frames

$$
\begin{aligned}
\widetilde{\mathbf{e}}_{\alpha} & =\left(\widetilde{\mathbf{e}}_{i}=\frac{\partial}{\partial x^{i}}-\widetilde{N}_{i}^{a}(x, y) \frac{\partial}{\partial y^{a}}, e_{b}=\frac{\partial}{\partial y^{b}}\right) ; \\
\widetilde{\mathbf{e}}^{\alpha} & =\left(\widetilde{e}^{i}=d x^{i}, \widetilde{\mathbf{e}}^{a}=d y^{a}+\widetilde{N}_{i}^{a}(x, y) d x^{i}\right) ; \text { and } \\
\widetilde{\mathbf{e}}_{\alpha} & =\left({ }^{\prime} \widetilde{\mathbf{e}}_{i}=\frac{\partial}{\partial x^{i}}-{ }^{\prime} \widetilde{N}_{i a}(x, p) \frac{\partial}{\partial p_{a}},{ }^{\prime} e^{b}=\frac{\partial}{\partial p_{b}}\right) ; \\
\widetilde{\mathbf{e}}^{\alpha} & =\left({ }^{\prime} e^{i}=d x^{i}, ' \mathbf{e}_{a}=d p_{a}+{ }^{\prime} \widetilde{N}_{i a}(x, p) d x^{i}\right) .
\end{aligned}
$$

Such a canonical N-splitting $\widetilde{\mathbf{N}}: T T V=h T V \oplus v T V$ and ' $\widetilde{\mathbf{N}}: T T^{*} V=h T^{*} V \oplus v T^{*} V$ is stated by respective generating Lagrange and/or Hamilton functions on any tangent and/or cotangent Lorentz bundle. The nonholonomic structure of phase spaces can be described in equivalent forms using canonical data $\left(\widetilde{L}, \widetilde{\mathbf{N}} ; \widetilde{\mathbf{e}}_{\alpha}, \widetilde{\mathbf{e}}^{\alpha}\right)$, with effective Largange density $\widetilde{L}$ (correspondingly, $\left(\widetilde{H}, ' \widetilde{\mathbf{N}} ; \quad \widetilde{\mathbf{e}}_{\alpha}, \widetilde{\mathbf{e}}^{\alpha}\right)$, with effective Hamilton density $\widetilde{H}$ ). We can consider a general N-splitting without effective Lagrangians (Hamiltonians), i.e. in terms of arbitrary geometric data $\left(\mathbf{N} ; \mathbf{e}_{\alpha}, \mathbf{e}^{\alpha}\right)$ (correspondingly ( ' $\mathbf{N}$; ' $\left.\mathbf{e}_{\alpha},{ }^{\prime} \mathbf{e}^{\alpha}\right)$ ). ${ }^{5}$ Using tensor products

5 On nonholonomic (co) tangent bundles, we can consider d-vectors if they are written in a form adapted to a prescribed N-connection structure, for instance,

$$
\begin{aligned}
\mathbf{X} & =\widetilde{\mathbf{X}}^{\alpha} \widetilde{\mathbf{e}}_{\alpha}=\widetilde{\mathbf{X}}^{i} \widetilde{\mathbf{e}}_{i}+X^{b} e_{b}=\mathbf{X}^{\alpha} \mathbf{e}_{\alpha}=\mathbf{X}^{i} \mathbf{e}_{i}+X^{b} e_{b} \in T \mathbf{T V}, \\
{ }^{\prime} \mathbf{X} & ={ }^{\prime} \widetilde{\mathbf{X}}^{\alpha} \widetilde{\mathbf{e}}_{\alpha}=\widetilde{\mathbf{X}}^{i}{ }^{\prime} \tilde{\mathbf{e}}_{i}+{ }^{\prime} X_{b}{ }^{\prime} e^{b}={ }^{\prime} \mathbf{X}^{\alpha} \mathbf{e}_{\alpha} \\
& =\mathbf{e}^{\prime} \mathbf{X}^{i} \mathbf{e}_{i}+{ }^{\prime} X_{b}{ }^{\prime} e^{b} \in T \mathbf{T}^{*} \mathbf{V} .
\end{aligned}
$$

Such formulas can be written equivalently for decompositions with respect to canonical, or arbitrary, $\mathrm{N}$-adapted bases. In brief, the $\mathrm{h}-\mathrm{v}$ and/or h-cv decompositions can be written $\mathbf{X}^{\alpha}=\widetilde{\mathbf{X}}^{\alpha}=\left(\widetilde{\mathbf{X}}^{i}, X^{b}\right)=$ $\left(\mathbf{X}^{i}, X^{b}\right),{ }^{\prime} \mathbf{X}^{\alpha}={ }^{\prime} \widetilde{\mathbf{X}}^{\alpha}=\left({ }^{\prime} \widetilde{\mathbf{X}}^{i},{ }^{\prime} X_{b}\right)=\left({ }^{\prime} \mathbf{X}^{i},{ }^{\prime} X_{b}\right)$. Considering $\mathbf{X}$ and ' $\mathbf{X}$ as 1-forms, we have

$$
\begin{aligned}
\mathbf{X} & =\widetilde{\mathbf{X}}_{\alpha} \mathbf{e}^{\alpha}=X_{i} e^{i}+\widetilde{\mathbf{X}}^{a} \widetilde{\mathbf{e}}_{a}=\widetilde{\mathbf{X}}_{\alpha} \mathbf{e}^{\alpha}=X_{i} e^{i}+\mathbf{X}^{a} \mathbf{e}_{a} \in T^{*} \mathbf{T V} \\
{ }^{\prime} \mathbf{X} & ={ }^{\prime} \widetilde{\mathbf{X}}_{\alpha} \mathbf{e}^{\prime} \mathbf{e}^{\alpha}={ }^{\prime} X_{i}{ }^{\prime} e^{i}+{ }^{\prime} \widetilde{\mathbf{X}}^{a}{ }^{\prime} \widetilde{\mathbf{e}}_{a}={ }^{\prime} \widetilde{\mathbf{X}}_{\alpha}{ }^{\prime} \mathbf{e}^{\alpha} \\
& ={ }^{\prime} X_{i}{ }^{\prime} e^{i}+{ }^{\prime} \mathbf{X}^{a}{ }^{\prime} \mathbf{e}_{a} \in T^{*} \mathbf{T}^{*} \mathbf{V},
\end{aligned}
$$

or, in brief, $\mathbf{X}_{\alpha}=\widetilde{\mathbf{X}}_{\alpha}=\left(X_{i}, \widetilde{\mathbf{X}}^{a}\right)=\left(X_{i}, \mathbf{X}^{a}\right), \quad \mathbf{X}_{\alpha}=\widetilde{\mathbf{X}}_{\alpha}=$ $\left({ }^{\prime} X_{i}, \widetilde{\mathbf{X}}^{a}\right)=\left({ }^{\prime} X_{i},{ }^{\prime} \mathbf{X}^{a}\right)$. of $\mathrm{N}$-adapted (co) frames on phase space, we can parameterize in $\mathrm{N}$-adapted forms (canonical or general ones) arbitrary tensors fields (d-tensors), connections and d-connections and other types of geometric objects, d-objects.

\subsubsection{Canonical d-metric and almost complex structures}

There are canonical data $\left(\widetilde{L}, \widetilde{\mathbf{N}} ; \widetilde{\mathbf{e}}_{\alpha}, \widetilde{\mathbf{e}}^{\alpha} ; \widetilde{g}_{j k}, \widetilde{g}^{j k}\right)$ and/or $\left(\widetilde{H}, \quad \widetilde{\mathbf{N}} ; \quad \widetilde{\mathbf{e}}_{\alpha}, \quad \widetilde{\mathbf{e}}^{\alpha} ; \quad\right.$ ' $\left.\widetilde{g}^{a b}, \quad{ }^{\prime} \widetilde{g}_{a b}\right)$ when the d-metrics are parameterized in the Hessian form both for the $\mathrm{h}$ - and (c)vcomponents,

$$
\begin{aligned}
& \widetilde{\mathbf{g}}=\widetilde{\mathbf{g}}_{\alpha \beta}(x, y) \widetilde{\mathbf{e}}^{\alpha} \otimes \widetilde{\mathbf{e}}^{\beta}=\widetilde{g}_{i j}(x, y) e^{i} \otimes e^{j} \\
& +\widetilde{g}_{a b}(x, y) \widetilde{\mathbf{e}}^{a} \otimes \widetilde{\mathbf{e}}^{a} \text { and/or } \\
& ' \widetilde{\mathbf{g}}={ }^{\prime} \widetilde{\mathbf{g}}_{\alpha \beta}(x, p)^{\prime} \widetilde{\mathbf{e}}^{\alpha} \otimes{ }^{\prime} \widetilde{\mathbf{e}}^{\beta}={ }^{\prime} \widetilde{g}_{i j}(x, p) e^{i} \otimes e^{j} \\
& +{ }^{\prime} \widetilde{g}^{a b}(x, p){ }^{\prime} \widetilde{\mathbf{e}}_{a} \otimes \mathbf{\mathbf { e }}_{b} .
\end{aligned}
$$

By frame transforms, the canonical d-metric structures (14) and (15) [with tildes] can be written, respectively, in general d-metric forms (9) and (10) [without tildes]. In explicit form, the general vierbein transforms are written $e_{\alpha}=e^{\underline{\alpha}}(u) \partial / \partial u^{\underline{\alpha}}$ and $e^{\beta}=e_{\beta}^{\beta}(u) d u^{\underline{\beta}}$. We underline the local coordinate indices in order to distinguish them from arbitrary abstract ones. In such formulas, the matrix $e^{\beta}{ }_{\underline{\beta}}$ is inverse to $e^{\underline{\alpha}}$ for orthonormalized bases. For Hamilton like configurations on cotangent bundles, we consider ${ }^{\prime} e_{\alpha}={ }^{\prime} e^{\underline{\alpha}}\left({ }^{\prime} u\right) \partial / \partial{ }^{\prime} u^{\underline{\alpha}}$ and ' $e^{\beta}={ }^{\prime} e^{\beta}{ }_{\beta}\left({ }^{\prime} u\right) d^{\prime} u^{\underline{\beta}}$. There are not used boldface symbols for such transforms because they can be not adapted to a $\mathrm{N}$-connection structure.

Using (13), respectively, for (9) and (10) and regrouping with respect to local coordinate bases, we prove that with respect to local coordinate frames, any d-metric structures on $\mathbf{T V}$ and/or $\mathbf{T}^{*} \mathbf{V}$,

$$
\begin{aligned}
& \mathbf{g}=\mathbf{g}_{\alpha \beta}(x, y) \mathbf{e}^{\alpha} \otimes \mathbf{e}^{\beta}=g_{\alpha} \underline{\beta}(x, y) d u^{\underline{\alpha}} \otimes d u^{\underline{\beta}} \text { and } / \text { or } \\
& { }^{\prime} \mathbf{g}={ }^{\prime} \mathbf{g}_{\alpha \beta}(x, p)^{\prime} \mathbf{e}^{\alpha} \otimes \mathbf{e}^{\prime}={ }^{\prime} g_{\underline{\alpha} \underline{\beta}}(x, p) d^{\prime} u^{\underline{\alpha}} \otimes d^{\prime} u^{\prime} \underline{\underline{\beta}} .
\end{aligned}
$$

These formulas can be subjected to frame transforms, $\mathbf{g}_{\alpha \beta}=$ $e^{\underline{\alpha}} e^{\underline{\beta}} g_{\underline{\alpha}} \underline{\beta}$ and ' $\mathbf{g}_{\alpha \beta}={ }^{\prime} e^{\underline{\alpha}}{ }^{\prime} e^{\underline{\beta}}{ }^{\prime} g_{\underline{\alpha} \underline{\beta}}$, and written in equivalent off-diagonal forms:

$$
\begin{aligned}
& g_{\underline{\alpha} \underline{\beta}}=\left[\begin{array}{cc}
g_{i j}(x)+g_{a b}(x, y) N_{i}^{a}(x, y) N_{j}^{b}(x, y) & g_{a e}(x, y) N_{j}^{e}(x, y) \\
g_{b e}(x, y) N_{i}^{e}(x, y) & g_{a b}(x, y)
\end{array}\right] \text { and/or }
\end{aligned}
$$

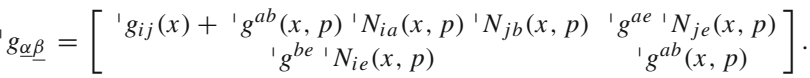

Parameterizations of type (16) for metrics are considered, for instance, in Kaluza-Klein theories on associated vector bundles. In our cases, the constructions are on (co) tangent bundles for geometric mechanics models. We conclude that if we fix a metric structure of type ' $\widetilde{\mathbf{g}}(15)$, we can elaborate equivalent models with ' $\mathbf{g}$ (10) determined by certain classes of nonholonomic frame transforms. Inversely, prescribing a 
d-metric ' $\mathbf{g}$, we can define nonholonomic variables when this metric structure can be represented as a $\widetilde{\mathbf{g}}$, i.e. in mechanical like variables, when ' $\mathbf{g}={ }^{\prime} \widetilde{\mathbf{g}}$. In a more general context, we can elaborate on bi-metric (and even multi-metric theories of gravity, geometric mechanics and thermodynamics) if we consider that ' $\widetilde{\mathbf{g}}$ and ' $\mathbf{g}$ are related via certain generalized nonholonomic transforms, see details an references in [34, 35].

The canonical N-connections $\widetilde{\mathbf{N}}$ and ' $\widetilde{\mathbf{N}}$ define respectively certain canonical almost complex structures $\widetilde{\mathbf{J}}$, on $\mathbf{T V}$, and $\mathbf{I}$, on $\mathbf{T}^{*} \mathbf{V}$. This follows, for instance, from such a construction on $\mathbf{T}^{*} \mathbf{V}$. Let us consider a linear operator ${ }^{\prime} \widetilde{\mathbf{J}}$ acting on ' $\mathbf{e}_{\alpha}=\left({ }^{\prime} \mathbf{e}_{i},{ }^{\prime} e^{b}\right)$ using formulas $\tilde{}{ }^{\prime}\left(\mathbf{e}_{i}\right)=-{ }^{\prime} e^{n+i}$ and ' $\widetilde{\mathbf{J}}\left({ }^{\prime} e^{n+i}\right)={ }^{\prime} \mathbf{e}_{i}$. This ' $\widetilde{\mathbf{J}}$ defines globally an almost complex structure ( $\left(\widetilde{\mathbf{J}} \circ{ }^{\prime} \widetilde{\mathbf{J}}=-\mathbf{I}\right.$, where $\mathbf{I}$ is the unity matrix) on $\mathbf{T}^{*} \mathbf{V}$. Such an operator is completely determined for Hamilton spaces by a $\widetilde{H}(x, p)$.

We note that $\widetilde{\mathbf{J}}$ and ' $\widetilde{\mathbf{J}}$ are standard almost complex structures only for the Euclidean signatures, respectively, on TV and $\mathbf{T}^{*} \mathbf{V}$. Contrary, we call them as pseudo almost complex structure. It is possible to omit tildes and write $\mathbf{J}$ and ' $\mathbf{J}$ for arbitrary frame/ coordinate transforms.

The canonical Neijenhuis tensor fields determined by Lagrange and Hamilton generating functions, for respective canonical almost complex structures $\widetilde{\mathbf{J}}$ on $\mathbf{T V}$ and/or $\widetilde{\mathbf{J}}$ on $\mathbf{T}^{*} \mathbf{V}$, are introduced as curvatures of respective $\mathrm{N}$ connections

$$
\begin{aligned}
& \widetilde{\Omega}(\tilde{\mathbf{X}}, \tilde{\mathbf{Y}}):=-[\tilde{\mathbf{X}}, \tilde{\mathbf{Y}}]+[\widetilde{\mathbf{J}} \widetilde{\mathbf{X}}, \widetilde{\mathbf{J}} \widetilde{\mathbf{Y}}]-\widetilde{\mathbf{J}}[\tilde{\mathbf{J}} \widetilde{\mathbf{X}}, \tilde{\mathbf{Y}}]-\tilde{\mathbf{J}}[\tilde{\mathbf{X}}, \tilde{\mathbf{J}} \widetilde{\mathbf{Y}}] \text { and/or } \\
& { }^{\prime} \widetilde{\Omega}\left({ }^{\prime} \widetilde{\mathbf{X}},{ }^{\prime} \tilde{\mathbf{Y}}\right):=-\left[{ }^{\prime} \widetilde{\mathbf{X}},{ }^{\prime} \tilde{\mathbf{Y}}\right]+\left[{ }^{\prime} \widetilde{\mathbf{J}} ' \widetilde{\mathbf{X}},{ }^{\prime} \widetilde{J}^{\prime} \tilde{\mathbf{Y}}\right] \\
& -{ }^{\prime} \widetilde{\mathbf{J}}\left[{ }^{\prime} \widetilde{\mathbf{J}} ' \widetilde{\mathbf{X}},{ }^{\prime} \tilde{\mathbf{Y}}\right]-{ }^{\prime} \widetilde{\mathbf{J}}\left[{ }^{\prime} \tilde{\mathbf{X}},{ }^{\prime} \widetilde{\mathbf{J}}{ }^{\prime} \tilde{\mathbf{Y}}\right],
\end{aligned}
$$

for any d-vectors $\mathbf{X}, \mathbf{Y}$ and ${ }^{\prime} \mathbf{X},{ }^{\prime} \mathbf{Y}$. Such formulas can be written in general form without tilde values if there are considered arbitrary frame transforms. In local form, a Nconnection on $\mathbf{T V}$, or $\mathbf{T}^{*} \mathbf{V}$, is characterized by such coefficients of (17) (i.e. the $\mathrm{N}$-connection curvature):

$$
\begin{aligned}
\Omega_{i j}^{a}= & \frac{\partial N_{i}^{a}}{\partial x^{j}}-\frac{\partial N_{j}^{a}}{\partial x^{i}}+N_{i}^{b} \frac{\partial N_{j}^{a}}{\partial y^{b}}-N_{j}^{b} \frac{\partial N_{i}^{a}}{\partial y^{b}}, \text { or } \\
{ }^{\prime} \Omega_{i j a}= & \frac{\partial^{\prime} N_{i a}}{\partial x^{j}}-\frac{\partial^{\prime} N_{j a}}{\partial x^{i}} \\
& +{ }^{\prime} N_{i b} \frac{\partial^{\prime} N_{j a}}{\partial p_{b}}-{ }^{'} N_{j b} \frac{\partial^{\prime} N_{i a}}{\partial p_{b}} .
\end{aligned}
$$

Almost complex structures $\mathbf{J}$ and ' $\mathbf{J}$ transform into standard complex structures for Euclidean signatures if $\Omega=0$ and/or ' $\Omega=0$. For almost complex canonical structures, we can consider canonical forms with "tilde" values determined by $\tilde{\mathbf{N}}=\left\{\tilde{N}_{j}^{b}\right\}$ and ' $\widetilde{\mathbf{N}}=\left\{{ }^{\prime} \tilde{N}_{i a}\right\}$.

Applying a straightforward $\mathrm{N}$-adapted calculus using for-

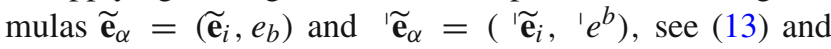
(18), we prove that the canonical nonholonomic frame structures on $\mathbf{T V}$ and/or $\mathbf{T}^{*} \mathbf{V}$ are characterized by corresponding anholonomy relations

$$
\begin{aligned}
{\left[\widetilde{\mathbf{e}}_{\alpha}, \widetilde{\mathbf{e}}_{\beta}\right] } & =\widetilde{\mathbf{e}}_{\alpha} \widetilde{\mathbf{e}}_{\beta}-\widetilde{\mathbf{e}}_{\beta} \widetilde{\mathbf{e}}_{\alpha}=\widetilde{W}_{\alpha \beta}^{\gamma} \widetilde{\mathbf{e}}_{\gamma} \text { and }\left[{ }^{\prime} \widetilde{\mathbf{e}}_{\alpha},{ }^{\prime} \widetilde{\mathbf{e}}_{\beta}\right] \\
& ={ }^{\prime} \widetilde{\mathbf{e}}_{\alpha}{ }^{\prime} \widetilde{\mathbf{e}}_{\beta}-{ }^{\prime} \widetilde{\mathbf{e}}_{\beta}{ }^{\prime} \widetilde{\mathbf{e}}_{\alpha}={ }^{1} \widetilde{W}_{\alpha \beta}^{\gamma}{ }^{\prime} \widetilde{\mathbf{e}}_{\gamma}
\end{aligned}
$$

with anholonomy coefficients $\widetilde{W}_{i a}^{b}=\partial_{a} \widetilde{N}_{i}^{b}, \widetilde{W}_{j i}^{a}=\widetilde{\Omega}_{i j}^{a}$, and ' $\widetilde{W}_{i b}^{a}=\partial^{\prime} \widetilde{N}_{i b} / \partial p_{a}$ and ' $\widetilde{W}_{j i a}={ }^{\prime} \widetilde{\Omega}_{i j a}$. We can define holonomic (integrable) frame configurations if the respective anholonomy coefficients in (19) are zero.

In geometric mechanics, the canonical d-metric structures $\widetilde{\mathbf{g}}$ (14) and ${ }^{\prime} \widetilde{\mathbf{g}}(15)$ are described by generic off-diagonal metrics (16) if respective anholonomy coefficients (19) are not trivial.

\subsection{Linear connections and curvatures for} Lagrange-Hamilton spaces

Elaborating on different type Lagrange-Hamilton models, we are not able to perform the constructions in $\mathrm{N}$ adapted anholonomic form if we work only with generalized (Finsler like) metrics determined by nonlinear quadratic forms $L(x, y)$ and/or $H(x, p)$ (6). The goal of this section is to analyze which classes of linear connections and respective covariant derivative operators can be generated canonically by fundamental generating functions.

\subsubsection{Distinguished connections, $N$-adapted distortions and curvatures}

We can define a linear connection $D$ on $\mathbf{T V}$ when a $\mathcal{L}$-duality between the tangent and corresponding cotangent bundles which can be defined by pull-back and push-forward maps. We omit geometric details on constructing such maps from/to base space to total space, considered, for instance, in [34, 35]. A linear connection ' $D$ on $\mathbf{T}^{*} \mathbf{V}$ is defined as follows: ${ }^{\prime} D_{{ } \mathbf{X}}{ }^{\prime} \mathbf{Y}:=\left(D_{\mathbf{X}} \mathbf{Y}\right)^{*}={ }^{\prime}\left(D_{\mathbf{X}} \mathbf{Y}\right)$, for any vector fields ${ }^{\prime} \mathbf{X}$ and ' $\mathbf{Y}$ on $\mathbf{T}^{*} \mathbf{V}$. Inversely, we can consider a linear connection

${ }^{\prime} D$ on $\mathbf{T}^{*} \mathbf{V}$ and then construct a linear connection ${ }^{\circ} D$ on

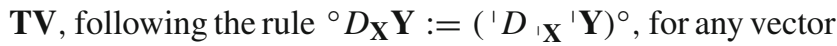
fields $\mathbf{X}$ and $\mathbf{Y}$ on $\mathbf{T V}$.

A distinguished connection (d-connection) is a linear connection $\mathbf{D}$ on $\mathbf{T V}$ ( or ' $\mathbf{D}$ on $\mathbf{T}^{*} \mathbf{V}$ ) which is compatible with the N-connection splitting (7).

The coefficients of d-connections can be defined and computed in corresponding $\mathrm{N}$-adapted forms,

$\mathbf{D}_{\mathbf{e}_{\beta}} \mathbf{e}_{\gamma}:=\Gamma_{\beta \gamma}^{\alpha} \mathbf{e}_{\alpha}$ and ${ }^{\prime} \mathbf{D}_{{ }^{\prime} \mathbf{e}_{\beta}}{ }^{\prime} \mathbf{e}_{\gamma}:={ }^{\prime} \Gamma_{\beta \gamma}^{\alpha}{ }^{\prime} \mathbf{e}_{\alpha}$.

For a h-v splitting, $\mathbf{D}_{\mathbf{e}_{k}} \mathbf{e}_{j}:=L^{i}{ }_{j k} \mathbf{e}_{i}, \mathbf{D}_{\mathbf{e}_{k}} e_{b}:=\dot{L}^{a}{ }_{b k} e_{a}, \mathbf{D}_{e_{c}} \mathbf{e}_{j}$ $:={ }_{C}^{i}{ }_{j c} \mathbf{e}_{i}, \mathbf{D}_{e_{c}} e_{b}:=C^{a}{ }_{b c} e_{a}$ and a h-cv splitting, ' $\mathbf{D}_{{ }_{1} \mathbf{e}_{k}}{ }^{\prime} \mathbf{e}_{j}:=$ ${ }^{\prime} L^{i}{ }_{j k}{ }^{\prime} \mathbf{e}_{i},{ }^{\prime} \mathbf{D}_{\mathbf{e}_{k}}{ }^{\prime} e^{b}:=-{ }^{\prime} L_{a k}^{b}{ }^{b} e^{a},{ }^{\prime} \mathbf{D}_{1} e^{c}{ }^{\prime} \mathbf{e}_{j}:={ }^{\prime} C^{i}{ }_{j}{ }^{c}{ }^{\prime} \mathbf{e}_{i}$, ${ }^{\prime} \mathbf{D} e^{c}{ }^{\prime} e^{b}:=-{ }^{\prime} C_{a}^{b c}{ }^{\prime} e^{a}$. In result, the $\mathrm{N}$-adapted coefficients of d-connections on (co) tangent Lorentz bundles can be 
parameterized (respectively) $\Gamma_{\beta \gamma}^{\alpha}=\left\{L^{i}{ }_{j k}, \dot{L}_{b k}^{a}, \dot{C}^{i}{ }_{j c}, C_{b c}^{a}\right\}$ and ' $\Gamma_{\beta \gamma}^{\alpha}=\left\{{ }^{\prime} L^{i}{ }_{j k},{ }^{\prime} L_{a k}^{b},{ }^{b} C^{i c}{ }_{j},{ }^{\prime} C_{a}{ }^{b c}\right\}$. These coefficients can be used for explicit computations of h- and/or v-splitting, cv-splitting, of covariant derivatives

$\mathbf{D}=\left({ }_{h} \mathbf{D},{ }_{v} \mathbf{D}\right) \quad$ and $\quad ' \mathbf{D}=\left({ }_{h}^{1} \mathbf{D},{ }_{v}^{i} \mathbf{D}\right)$,

where ${ }_{h} \mathbf{D}=\left\{L^{i}{ }_{j k}, \dot{L}_{b k}^{a}\right\},{ }_{v} \mathbf{D}=\left\{\dot{C}^{i}{ }_{j c}, C^{a}{ }_{b c}\right\}$ and ${ }_{h}^{1} \mathbf{D}=$ $\left\{{ }^{\prime} L^{i}{ }_{j k},{ }^{\prime} L_{a k}^{b}\right\},{ }_{v}^{\prime} \mathbf{D}=\left\{{ }^{\prime} C^{i}{ }_{j}^{c},{ }^{\prime} C_{a}^{b c}\right\}$.

We can consider a linear connection $\underline{D}$ (which is not obligatory a d-connection) and a d-connection $\mathbf{D}$ both defined on TV. Such geometric objects are respectively denoted ' $\underline{D}$ and ' $\mathbf{D}$ on $\mathbf{T}^{*} \mathbf{V}$. For (co)vector bundles, there are nonholonomic relations with respective distortion d-tensors $\mathbf{Z}:=\mathbf{D}-\underline{D}$ and ${ }^{\prime} \mathbf{Z}:={ }^{\prime} \mathbf{D}-{ }^{\prime} \underline{D}$.

Using similar definitions and theorems both for linear connections and d-connections, we prove that d-connection $\mathbf{D}$, or ' $\mathbf{D}$, is characterized by respective curvature $(\mathcal{R}$, or ' $\mathcal{R})$, torsion $(\mathcal{T}$, or ' $\mathcal{T})$, nonmetricity, $(\mathcal{Q}$, or ' $\mathcal{Q})$, d-tensors,

$$
\begin{aligned}
& \mathcal{R}(\mathbf{X}, \mathbf{Y}):=\mathbf{D}_{\mathbf{X}} \mathbf{D}_{\mathbf{Y}}-\mathbf{D}_{\mathbf{Y}} \mathbf{D}_{\mathbf{X}}-\mathbf{D}_{[\mathbf{X}, \mathbf{Y}]}, \mathcal{T}(\mathbf{X}, \mathbf{Y}) \\
& :=\mathbf{D}_{\mathbf{X}} \mathbf{Y}-\mathbf{D}_{\mathbf{Y}} \mathbf{X}-[\mathbf{X}, \mathbf{Y}], \mathcal{Q}(\mathbf{X}):=\mathbf{D}_{\mathbf{X}} \mathbf{g} \text {, or } \\
& { }^{\prime} \mathcal{R}\left({ }^{\prime} \mathbf{X},{ }^{\prime} \mathbf{Y}\right):={ }^{\prime} \mathbf{D}_{{ } \mathbf{X}}{ }^{\prime} \mathbf{D}_{{ }^{\prime} \mathbf{Y}}-{ }^{\prime} \mathbf{D}_{{ } \mathbf{Y}}{ }^{\prime} \mathbf{D}_{{ }^{\prime} \mathbf{X}}-{ }^{\prime} \mathbf{D}_{\left[{ }^{\prime} \mathbf{X},{ }^{\prime} \mathbf{Y}\right]} \text {, } \\
& { }^{\prime} \mathcal{T}\left({ }^{\prime} \mathbf{X},{ }^{\prime} \mathbf{Y}\right):={ }^{\prime} \mathbf{D}_{{ } \mathbf{X}}{ }^{\prime} \mathbf{Y}-{ }^{\prime} \mathbf{D}_{{ } \mathbf{Y}}{ }^{\prime} \mathbf{X}-\left[{ }^{\prime} \mathbf{X},{ }^{\prime} \mathbf{Y}\right],
\end{aligned}
$$

and ${ }^{\prime} \mathcal{Q}\left({ }^{\prime} \mathbf{X}\right):={ }^{\prime} \mathbf{D}{ } \mathbf{X}{ }^{\prime} \mathbf{g}$. The $\mathrm{N}$-adapted coefficients for the curvature, torsion and nonmetricity d-tensors are provided in Appendices to [34,35], see also references therein. The geometric d-tensors (20) are written, for instance, using tilde on symbols if such d-objects are defined and computed for Lagrange (or Hamilton) generating functions, see below.

\subsubsection{The Ricci and Einstein d-tensors on phase spaces and (co) vector bundles}

Respectively, the Ricci d-tensors are defined and computed as $R i c=\left\{\mathbf{R}_{\alpha \beta}:=\mathbf{R}_{\alpha \beta \tau}^{\tau}\right\}$, for a d-connection $\mathbf{D}$, and ${ }^{\prime}$ Ric $=\left\{{ }^{\prime} \mathbf{R}_{\alpha \beta}:={ }^{\prime} \mathbf{R}^{\tau}{ }_{\alpha \beta \tau}\right\}$, for a d-connection $' \mathbf{D}$, see formulas (20). In $\mathrm{N}$-adapted form, we prove that the $\mathrm{N}$-adapted coefficients of the Riccid-tensors of a d-connection $\mathbf{D}$ (or ' $\mathbf{D}$ )

$$
\begin{aligned}
& {[\mathbf{g}, \mathbf{N}] \simeq[\widetilde{\mathbf{g}}, \widetilde{\mathbf{N}}] \simeq[\widetilde{\theta}:=\widetilde{\mathbf{g}}(\widetilde{\mathbf{J}} \cdot, \cdot), \widetilde{\mathbf{P}}, \widetilde{\mathbf{J}}, \widetilde{J}]} \\
& \Longrightarrow\left\{\begin{array}{lcc}
\nabla: & \nabla \mathbf{g}=0 ; \mathbf{T}[\nabla]=0, & \text { Lagrange LC-connection; } \\
\widehat{\mathbf{D}}: & \widehat{\mathbf{D}} \mathbf{g}=0 ; h \widehat{\mathbf{T}}=0, v \widehat{\mathbf{T}}=0 . & \text { canonical Lagrange d-connection; } \\
\widetilde{\mathbf{D}}: & \widetilde{\mathbf{D}} \widetilde{\theta}=0, \widetilde{\mathbf{D}} \widetilde{\theta}=0 & \text { almost sympl. Lagrange d-connection; }
\end{array}\right.
\end{aligned}
$$$$
\begin{aligned}
& { }_{s} R:=\mathbf{g}^{\alpha \beta} \mathbf{R}_{\alpha \beta}=g^{i j} R_{i j}+g^{a b} R_{a b}=R+S, \text { or } \\
& { }_{s} R:={ }^{\prime} \mathbf{g}^{\alpha \beta}{ }^{\prime} \mathbf{R}_{\alpha \beta}={ }^{\prime} g^{i j}{ }^{i} R_{i j}+{ }^{\prime} g^{a b}{ }^{\prime} R_{a b}={ }^{\prime} R+{ }^{'} S,
\end{aligned}
$$

If a phase space is enabled both with a d-connection, $\mathbf{D}$ (or 'D), and d-metric, g (9) (or ' $\mathbf{g}$ (10)) [in particular, we can consider canonical "tilde" values with d-metrics $\widetilde{\mathbf{g}}$ (14) and/or ' $\widetilde{\mathbf{g}}(15)$, and their frame transforms], we can define and compute nonholonomic Ricci scalars. In result, we obtain that the scalar curvature of a d-connection $\mathbf{D}$, or ' $\mathbf{D}$, can be defined and computed for the inverse d-metric $\mathbf{g}^{\alpha \beta}$, or ${ }^{\prime} \mathbf{g}^{\alpha \beta}$,

with respective h- and v-components $R=g^{i j} R_{i j}, S=$ $g^{a b} S_{a b}$, or ' $R={ }^{\prime} g^{i j}{ }^{\prime} R_{i j},{ }^{\prime} S={ }^{\prime} g_{a b}{ }^{\prime} S^{a b}$.

By constructions, the Einstein d-tensors on $\mathbf{T V}$ and/or $\mathbf{T}^{*} \mathbf{V}$ are defined:

$$
\begin{aligned}
E n & =\left\{\mathbf{E}_{\alpha \beta}:=\mathbf{R}_{\alpha \beta}-\frac{1}{2} \mathbf{g}_{\alpha \beta}{ }_{s} R\right\} \text { and/or } \\
{ }^{\prime} E n & =\left\{{ }^{\prime} \mathbf{E}_{\alpha \beta}:={ }^{\prime} \mathbf{R}_{\alpha \beta}-\frac{1}{2}{ }^{\prime} \mathbf{g}_{\alpha \beta}{ }_{s}^{\prime} R\right\} .
\end{aligned}
$$

Such values can be used in MGTs and encoding geometric and physical models in quantum computing theories.

\subsubsection{Physically important d-connections for geometric mechanics}

The Lagrange and/or Hamilton phase spaces (with a possible $\mathcal{L}$-duality) can be endowed and characterized respectively by different type geometric and physically important linear connections and canonical/ almost symplectic connections, which are equivalent if respective distorting relations are postulated. In our approaches to geometric mechanics and classical and quantum field/ thermodynamic and gravity theories, we use such linear connection structures:

$\left[{ }^{\prime} \mathbf{g},{ }^{\prime} \mathbf{N}\right] \simeq\left[{ }^{\prime} \widetilde{\mathbf{g}},{ }^{\prime} \widetilde{\mathbf{N}}\right] \simeq\left[{ }^{\prime} \tilde{\theta}:=' \widetilde{\mathbf{g}}\left({ }^{\prime} \widetilde{\mathbf{J}}, \cdot\right),{ }^{\prime} \widetilde{\mathbf{P}},{ }^{\prime} \widetilde{\mathbf{J}},{ }^{\prime} \widetilde{\mathbb{J}}\right]$

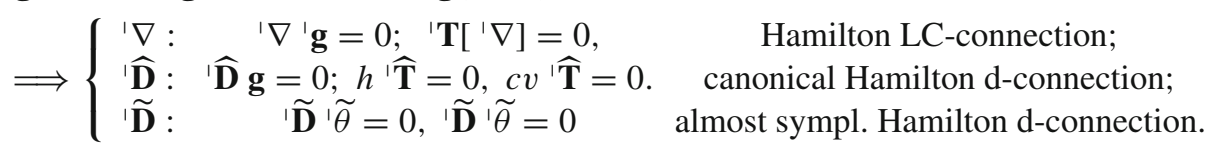


We can consider distortion relations

$$
\begin{aligned}
\widehat{\mathbf{D}}= & \nabla+\widehat{\mathbf{Z}}, \widetilde{\mathbf{D}}=\nabla+\widetilde{\mathbf{Z}}, \text { and } \widehat{\mathbf{D}}=\widetilde{\mathbf{D}} \\
& +\mathbf{Z}, \text { determined by }(\mathbf{g}, \mathbf{N}) ; \\
{ }^{\prime} \widehat{\mathbf{D}}= & { }^{\prime} \nabla+{ }^{\prime} \widehat{\mathbf{Z}},{ }^{\prime} \widetilde{\mathbf{D}}={ }^{\prime} \nabla+{ }^{\prime} \widetilde{\mathbf{Z}}, \text { and }{ }^{\prime} \widehat{\mathbf{D}}={ }^{\prime} \widetilde{\mathbf{D}} \\
& +{ }^{\prime} \mathbf{Z}, \text { determined by }\left({ }^{\prime} \mathbf{g},{ }^{\prime} \mathbf{N}\right) ;
\end{aligned}
$$

with distortion d-tensors $\widehat{\mathbf{Z}}, \widetilde{\mathbf{Z}}$, and $\mathbf{Z}$, on $T \mathbf{T V}$, and $\widehat{\mathbf{Z}}$, ' $\widetilde{\mathbf{Z}}$, and ${ }^{~} \mathbf{Z}$, on $T \mathbf{T}^{*} \mathbf{V}$.

Geometric mechanic models are characterized by respective canonical and/or almost symplectic distortion d-tensors $\widehat{\mathbf{Z}}[\widetilde{\mathbf{g}}, \widetilde{\mathbf{N}}], \widetilde{\mathbf{Z}}[\widetilde{\mathbf{g}}, \widetilde{\mathbf{N}}]$, and $\mathbf{Z}[\widetilde{\mathbf{g}}, \widetilde{\mathbf{N}}]$, for (almost symplectic)

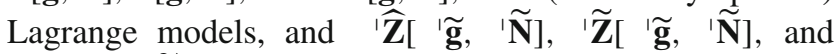
$' \mathbf{Z}\left[{ }^{\prime \mathbf{g}}, \quad ' \widetilde{\mathbf{N}}\right]$, for (almost symplectic) Hamilton models. Respective phase space geometries can be described in equivalent forms by such data

$$
\text { on TV : } \quad \begin{aligned}
&(\mathbf{g}, \mathbf{N}, \widehat{\mathbf{D}}) \leftrightarrows(L: \widetilde{\mathbf{g}}, \widetilde{\mathbf{N}}, \widetilde{\mathbf{D}}) \\
& \mathfrak{1} \text { possible } \mathcal{L} \text {-duality }
\end{aligned}
$$$$
\text { on } \mathbf{T}^{*} \mathbf{V} \cdot\left({ }^{\prime} \mathbf{g}, \mid \mathbf{N}, ' \widehat{\mathbf{D}}\right) \leftrightarrows\left(H:, \widetilde{\mathbf{g}},{ }^{\prime} \widetilde{\mathbf{N}}\right.
$$

with respective distortion d-tensors $\widehat{\mathcal{Z}}$, on $\mathbf{T V}$, and ' $\widehat{\mathcal{Z}}$, on $\mathbf{T}^{*} \mathbf{V}$. Similarly, we obtain for the Ricci d-tensors,

$$
\begin{aligned}
\widehat{R} i c[\mathbf{g}, \widehat{\mathbf{D}} & =\nabla+\widehat{\mathbf{Z}}]=\operatorname{Ric}[\mathbf{g}, \nabla]+\widehat{Z} i c[\mathbf{g}, \widehat{\mathbf{Z}}],{ }^{\prime} \widehat{R} i c\left[{ }^{\prime} \mathbf{g},{ }^{\prime} \widehat{\mathbf{D}}\right. \\
& \left.={ }^{\prime} \nabla+{ }^{\prime} \widehat{\mathbf{Z}}\right]={ }^{\prime} \operatorname{Ric}\left[{ }^{\prime} \mathbf{g},{ }^{\prime} \nabla\right]+{ }^{\prime} \widehat{Z} i c\left[{ }^{\prime} \mathbf{g},{ }^{\prime} \widehat{\mathbf{Z}}\right],
\end{aligned}
$$

with respective distortion d-tensors $\widehat{Z} i c$, on $\mathbf{T V}$, and ' $\widehat{Z} i c$, on $\mathbf{T}^{*} \mathbf{V}$. Finally, for the scalar curvature of canonical dconnection $\widehat{\mathbf{D}}$, or ' $\widehat{\mathbf{D}}$,

$$
\begin{aligned}
{ }_{s} \widehat{R}[\mathbf{g}, \widehat{\mathbf{D}} & =\nabla+\widehat{\mathbf{Z}}]=\mathcal{R}[\mathbf{g}, \nabla]+{ }_{s} \widehat{Z}[\mathbf{g}, \widehat{\mathbf{Z}}],{ }_{s}^{\prime} \widehat{R}\left[{ }^{\prime} \mathbf{g}, ' \widehat{\mathbf{D}}\right. \\
& \left.={ }^{\prime} \nabla+{ }^{\prime} \widehat{\mathbf{Z}}\right]={ }_{s}^{1} R\left[{ }^{\prime} \mathbf{g},{ }^{\prime} \nabla\right]+{ }_{s} \widehat{Z}\left[{ }^{\prime} \mathbf{g}, ' \widehat{\mathbf{Z}}\right],
\end{aligned}
$$

with respective distortion scalar functionals ${ }_{s} \widehat{Z}$, on $\mathbf{T V}$, and ${ }_{s}^{1} \widehat{Z}$, on $\mathbf{T}^{*} \mathbf{V}$.

Above formulas can be reformulated for distortions of the almost symplectic Lagrange, or Finsler, d-connections, for instance, considering

$$
\begin{aligned}
& \widetilde{\mathcal{R}}[\widetilde{\mathbf{g}} \simeq \widetilde{\theta}, \widetilde{\mathbf{D}}=\nabla+\widetilde{\mathbf{Z}}]=\mathcal{R}[\widetilde{\mathbf{g}} \simeq \widetilde{\theta}, \nabla]+\widetilde{\mathcal{Z}}[\widetilde{\mathbf{g}} \simeq \widetilde{\theta}, \widetilde{\mathbf{Z}}], \\
& { }^{\prime} \widetilde{\mathcal{R}}\left[\widetilde{\mathbf{g}} \simeq{ }^{\prime} \widetilde{\theta}, ' \widetilde{\mathbf{D}}={ }^{\prime} \nabla+{ }^{\prime} \widetilde{\mathbf{Z}}\right]={ }^{\prime} \mathcal{R}\left[' \widetilde{\mathbf{g}} \simeq{ }^{\prime} \widetilde{\theta},{ }^{\prime} \nabla\right]+{ }^{\prime} \widetilde{\mathcal{Z}}\left[{ }^{\prime} \mathbf{g} \simeq{ }^{\prime} \widetilde{\theta}, ' \widetilde{\mathbf{Z}}\right],
\end{aligned}
$$

We can work with canonical d-connection structures on (co) tangent bundles, $\widehat{\mathbf{D}}$ and/or ' $\widehat{\mathbf{D}}$ which allows us to decouple and integrate in most general exact and parametric forms certain effective geometric flow and/or modified gravitational field equations. Here we note that Lagrange-Finsler variables can be introduced on 4-d, and higher dimension, (pseudo) Riemannian spaces and in GR if nonholonomic fibered structures are considered on spacetime manifolds, see discussions and examples in Refs. [24-29,34,35].

An important example is that when imposing certain (in general, nonholonomic) constraints of type $\widehat{\mathbf{Z}}=0$, we obtain $\widehat{\mathbf{D}}_{\mid \mathbf{Z}=0} \simeq \nabla$ even $\widehat{\mathbf{D}} \neq \nabla$. If such conditions are satisfied, we can extract (pseudo) Riemannian or effective geometric mechanical (with tilde values) LC-configurations from more (general) nonholonmic metric-affine structures. For instance, we can obtain LC-configurations for geometric models with $\widehat{\mathbf{D}}$ and/or $\widehat{\mathbf{D}}$ for respective zero distortions, $\widehat{\mathbf{Z}}$ and/or $\widehat{\mathbf{Z}}$. Equivalently, one can be considered the zero torsion conditions for $\widehat{\mathcal{T}}=\left\{\widehat{\mathbf{T}}_{\alpha \beta}^{\gamma}\right\}=0$ and/or $\quad \widehat{\mathcal{T}}=\left\{{ }^{\prime} \widehat{\mathbf{T}}_{\alpha \beta}^{\gamma}\right\}=0$.

Using distortions of linear connections, we can prove in abstract and $\mathrm{N}$-adapted forms that there are canonical distortion relations encoding generating functions for respective Lagrange-Hamilton and equivalent nonholonomic variables: For the curvature d-tensors, we compute

$$
\begin{aligned}
\widehat{\mathcal{R}}[\mathbf{g}, \widehat{\mathbf{D}} & =\nabla+\widehat{\mathbf{Z}}]=\mathcal{R}[\mathbf{g}, \nabla]+\widehat{\mathcal{Z}}[\mathbf{g}, \widehat{\mathbf{Z}}],{ }^{\prime} \widehat{\mathcal{R}}\left[{ }^{\prime} \mathbf{g},{ }^{\prime} \widehat{\mathbf{D}}\right. \\
& \left.={ }^{\prime} \nabla+{ }^{\prime} \widehat{\mathbf{Z}}\right]={ }^{\prime} \mathcal{R}\left[{ }^{\prime} \mathbf{g},{ }^{\prime} \nabla\right]+{ }^{\prime} \widehat{\mathcal{Z}}\left[{ }^{\prime} \mathbf{g},{ }^{\prime} \widehat{\mathbf{Z}}\right],
\end{aligned}
$$

and any similar geometric objects with "tilde" symbols.

\section{Geometric flow evolution of classical mechanical systems}

The goal of this section is to formulate in canonical Hamilton variables the theory of nonholonomic geometric flows of relativistic mechanical systems. This is important for further developments in classical and quantum information theories when the Hamilton variables are used in explicit form. We shall present also the main results in canonical Lagrange variables because such formulas are very important for investigating various connections between quantum field theory, QFT, quantum gravity, QG, and quantum information theory. ${ }^{6}$

\footnotetext{
6 Such a research is related to author's project on geometric flows and applications in physics which was elaborated in 2005 for a sabbatical professor fellowship at CSIC, Madrid, in Spain, and further developments supported by a project IDEI, in Romania; and related visiting projects at CERN (Switzerland); M. Planck Institute, Munich, and A. Einstein Institute, Postdam, (Germany) etc. Those projects were on applications of nonholonomic geometric methods in classical and quantum mechanics and physics, with various generalizations to deformation quantization, noncommutative geometry etc. A subdirection of former research was devoted to studies on flow evolution of Lagrange-Hamilton systems geometrized on (co) tangent bundles, which resulted in a series of works on the nonholonomic geometric evolution of Finsler-Lagrange-Hamilton space spaces, see historical
} 
3.1 Relativistic geometric flows and Perelman's thermodynamics for phase spacetimes

Let us consider families of nonholonomic 8-d tangent and cotangent Lorentz bundles, $T \mathbf{V}(\tau)$ and $T^{*} \mathbf{V}(\tau)$ parameterized by a positive parameter $\tau, 0 \leq \tau \leq \tau_{0}$. Such phase spacetimes are enabled with corresponding sets of canonical d-metrics of type (14) and (15), $\widetilde{\mathbf{g}}(\tau)=\widetilde{\mathbf{g}}(\tau, u)$ and $\widetilde{\mathbf{g}}(\tau)=\mathbf{\mathbf { g }}\left(\tau,{ }^{\prime} u\right)$ and canonical N-connections of type (12), ${ }^{\prime} \widetilde{\mathbf{N}}(\tau)={ }^{\prime} \tilde{\mathbf{N}}\left(\tau,{ }^{\prime} u\right)$. Any relativistic nonholonomic phase spacetime $T \mathbf{V} \subset T \mathbf{V}(\tau)$ and/or $T^{*} \mathbf{V} \subset T^{*} \mathbf{V}(\tau)$ can be enabled with necessary types of double nonholonomic $(2+2)+(2+2)$ and $(3+1)+(3+1)$ splitting, see details for such geometric constructions in [24-28]. ${ }^{7}$ For instance, a nonholonomic $(3+1)+(3+1)$ splitting on a $T \mathbf{V}$ can be chosen in such a form that any open region on a base Lorentz manifold, $U \subset \mathbf{V}$, is covered by a family of 3-d spacelike hypersurfaces $\widehat{\Xi}_{t}$, or $\widetilde{\Xi}_{t}$, parameterized by a time like parameter $t$. The parameterizations of hypersurfaces can be labeled in certain forms which are adapted to the type of canonical d-connection we use for our geometric constructions. In this work, we prefer to use "tilde" labels/ values related to geometric mechanics. On a typical cofiber of $T^{*} \mathbf{V}$, we can consider a 3 -d cofiber hypersurface ${ }^{\prime} \widetilde{\Xi}_{E}$, for instance, of signature $(+++)$ with a label $E$ for parameterizations by an energy type parameter. We can write correspondingly $\widetilde{\Xi}=\left(\widetilde{\Xi}_{t}, \widetilde{\Xi}_{E}\right)$ and ' $\widetilde{\Xi}=\left(\widetilde{\Xi}_{t},{ }^{\prime} \widetilde{\Xi}_{E}\right)$ for nonholonomic distributions of base and fiber hypersurfaces with conventional splitting $3+3$ of signature $(+++;+++)$ on total phase space. For additional shell decompositions of type $(2+2)+(2+2)$, we can use also a $s$-label, ${ }_{s} \widehat{\Xi}=\left({ }_{s} \widehat{\Xi}_{t},{ }_{s} \widehat{\Xi}_{E}\right) \subset{ }_{s} T^{*} \mathbf{V}$, if we shall be interested in constructing certain classes of exact or parametric solutions of geometric flow equations. In general, we can elaborate on two generic different types of geometric phase flow theories: The fist type is with a conventional parameter $\tau(\chi)$ admitting re-parameterizations of a temperature like parameter used for labeling 4-d Lorentz spacetimes and their

Footnote 6 continued

remarks and a comprehensive bibliography in Appendix B.4.17 to Ref. [34]. Here we note that nonholonomic generalizations of Perelman functionals and Hamilton geometric evolution equations were considered for Finsler-Lagrange systems in Refs. [19,20], see further generalizations for almost Kähler-Lagrange-Hamilton models on Lie algebroids , relativistic Lagrange-Hamilton mechanics etc. [21,22,50]. In principle, Finsler-Lagrange-Hamilton variables can be introduced on any (non) commutative/(super) manifold, which allows to re-write in effective (super/noncommutative) mechanic forms all results on geometric flows of physical theories elaborated in [23-29], see also references therein.

7 Additionally to coordinate and index conventions from footnote 2, we label the local $(3+1)+(3+1)$ coordinates in the form ' $u=\left\{{ }^{\prime} u^{\alpha}=\right.$ ' $\left.u^{\alpha_{s}}=\left(x^{i_{1}}, y^{a_{2}} ; p_{a_{3}}, p_{a_{4}}\right)=\left(x^{\grave{\imath}}, u^{4}=y^{4}=t ; p_{\grave{a}}, p_{8}=E\right)\right\}$ for $i_{1}, j_{1}, k_{1}, \ldots=1,2 ; a_{1}, b_{1}, c_{1}, \ldots=3,4 ; a_{2}, b_{2}, c_{2}, \ldots=5,6$; $a_{3}, b_{3}, c_{3}, \ldots=7,8$; and $\grave{i}, \grave{j}, \grave{k}, \ldots=1,2,3$, respectively, $\grave{a}, \grave{b}, \grave{c}, \ldots=$ $5,6,7$ can be used for corresponding spacelike hyper surfaces on a base Lorentz manifold and typical cofiber. phase space configurations. The second type of models is with $\tau(t)$ as a time like parameter when $(3+3)$-d spacelike phase configurations evolve relativistically on a "redefined' time like coordinate. In this work, we elaborate on theories of type 1 .

\subsubsection{Perelman-Lagrange and Perelman-Hamilton functionals}

In [20], we studied geometric flows of Finsler-Lagrange theories using canonical data $(\mathbf{g}(\tau), \widetilde{\mathbf{D}}(\tau))$ when various generalizations and applications in MGTs were elaborated for the data $(\mathbf{g}(\tau), \widehat{\mathbf{D}}(\tau)),[19,23-29]$. Those constructions were based on nonholonomic generalizations of Perelman's functionals [1] and distortion relations form the Levi-Civita configurations $(\mathbf{g}(\tau), \nabla(\tau))$. Let us consider how Perelman's functionals can be generalized in relativistic form for geometric flow evolution of Lagrange-Hamilton spaces.

F- and W-functionals in canonical J. Lagrange variables: Considering canonical Lagrange data $(\widetilde{\mathbf{g}}(\tau), \widetilde{\mathbf{D}}(\tau))$ on tangent Lorentz bundles in order to postulate the functionals:

$$
\begin{aligned}
\widetilde{\mathcal{F}}= & \widetilde{\int} e^{-\tilde{f}} \sqrt{\left|\widetilde{\mathbf{g}}_{\alpha \beta}\right|} d^{8} u\left({ }_{s} \widetilde{R}+|\widetilde{\mathbf{D}} \widetilde{f}|^{2}\right) \text { and } \\
\widetilde{\mathcal{W}}= & \widetilde{\int} \tilde{\mu} \sqrt{\left|\widetilde{\mathbf{g}}_{\alpha \beta}\right|} d^{8} u\left[\tau \left({ }_{s} \widetilde{R}+\left|{ }_{h} \widetilde{\mathbf{D}} \widetilde{f}\right|\right.\right. \\
& \left.\left.+\left|{ }_{v} \widetilde{\mathbf{D}} \tilde{f}\right|\right)^{2}+\widetilde{f}-16\right],
\end{aligned}
$$

where the normalizing function $\tilde{f}(\tau, u)$ satisfies the conditions

$$
\widetilde{\int \mu} \sqrt{\left|\widetilde{\mathbf{g}}_{\alpha \beta}\right|} d^{8} u:=\int_{t_{1}}^{t_{2}} \int_{\widetilde{\Xi}_{t}} \int_{y_{(0)}^{8}}^{y^{8}} \int_{\widetilde{\Xi}_{E}} \tilde{\mu} \sqrt{\left|\widetilde{\mathbf{g}}_{\alpha \beta}\right|} d^{8} u=1
$$

for $\tilde{\mu}=(4 \pi \tau)^{-8} e^{-\tilde{f}}$, when the coefficients $16=2 \times 8$ is for 8 -d spaces. For 3-d hypersurface LC-configurations with $\nabla$, such values transform into the standard G. Perelman functionals. The Ricci scalar ${ }_{s} \widetilde{R}$ is taken for the Ricci d-tensor $\widetilde{\mathbf{R}}_{\alpha \beta}(21)$ constructed for the canonical Lagrange data $(\widetilde{\mathbf{g}}, \widetilde{\mathbf{D}})$. Re-defining the normalization functions and using corresponding nonholonomic frame transforms and d-connection distortions, we can re-write the functionals (26) and (27) in "hat" variables, $\widehat{\mathcal{F}}$ and $\widehat{\mathcal{W}}$, see similar constructions in [19,24-28].

F- and $W$-functionals in canonical $W$. Hamilton variables We use canonical data ( $\left.\widetilde{\mathbf{g}}(\tau),{ }^{\prime} \widetilde{\mathbf{D}}(\tau)\right)$ on cotangent Lorentz bundles and postulate the functionals:

$$
\begin{aligned}
& \text { ' } \widetilde{\mathcal{F}}=\tilde{\int}^{-{ }^{\prime} \tilde{f}} \sqrt{\left|\widetilde{\mathbf{g}}_{\alpha \beta}\right|} d^{8}{ }^{\prime} u\left({ }_{s}^{1} \widetilde{R}+\mid{ }^{\prime} \widetilde{\mathbf{D}}^{\prime} \tilde{f}^{2}\right) \text { and } \\
& { }^{\prime} \widetilde{\mathcal{W}}=\int^{\prime} \mid \tilde{\mu} \sqrt{\left|\widetilde{\mathbf{g}}_{\alpha \beta}\right|} d^{8}{ }^{\prime} u\left[\tau\left({ }_{s}^{1} \widetilde{R}+\left|{ }_{h}^{1} \widetilde{\mathbf{D}} \cdot \widetilde{f}\right|+\left|{ }_{v}^{1} \widetilde{\mathbf{D}} \cdot \widetilde{f}\right|\right)^{2}\right. \\
& +1 \tilde{f}-16] \text {, }
\end{aligned}
$$


where the normalizing function $\quad \tilde{f}\left(\tau,{ }^{\prime} u\right)$ satisfies

$$
\tilde{\int}\left|\tilde{\mu} \sqrt{\left|\widetilde{\mathbf{g}}_{\alpha \beta}\right|} d^{8} \cdot u:=\int_{t_{1}}^{t_{2}} \int_{\widetilde{\Xi}_{t}} \int_{E_{1}}^{E_{2}} \int_{\mid \widetilde{\Xi}_{E}}\right| \widetilde{\mu} \sqrt{\left|\widetilde{\mathbf{g}}_{\alpha \beta}\right|} d^{8} \mid u=1
$$

for $\quad \tilde{\mu}=(4 \pi \tau)^{-8} e^{-' \tilde{f}}$, when the coefficient $16=2 \times 8$ is taken for 8-d spaces. The Ricci scalar ${ }_{s}^{1} \widetilde{R}$ is taken for the Ricci d-tensor ' $\widetilde{\mathbf{R}}_{\alpha \beta}(22)$ constructed using the canonical Hamilton data ( $\widetilde{\mathbf{g}}, ' \widetilde{\mathbf{D}})$.

Similar functionals can be postulated for nonholonomic geometric flows on $T^{*} \mathbf{V}$ using data ( $\left.\mathbf{g}(\tau), \quad \widehat{\mathbf{D}}(\tau)\right)$ and redefined integration measures and normalizing functions on respective hypersurfaces. Considering LC-configurations with ${ }^{\prime} \widetilde{\mathbf{D}}_{\mid} \mid \widetilde{\mathbf{T}}=0={ }^{\prime} \nabla$ and/or ${ }^{\prime} \widehat{\mathbf{D}}_{\mid} \widehat{\mathbf{T}}_{=0}={ }^{\prime} \nabla$, the values (28) and (29) transform respectively into 8-d phase space versions of the so called Perelman's F-entropy and W-entropy. It should be noted that ' $\widetilde{\mathcal{W}}$ and/or ' $\widehat{\mathcal{W}}$ do not have a character of entropy for pseudo-Riemannian metrics but can be treated as a value characterizing relativistic geometric hydrodynamic phase space flows.

\section{Nonholonomic lapse and shift variables}

Using $\mathrm{N}$-adapted diadic shell and/or double $(2+2)+(2+2)$ and $(3+1)+(3+1)$ frame and coordinate transforms of metrics with additional dependence on a flow parameter, we can introduce various parameterizations of geometric objects on phase spacetimes. To define thermodynamic like variables for geometric flow evolution of stationary configurations on $T^{*} \mathbf{V}$, we take

$$
\begin{aligned}
& ' \mathbf{g}=\mathbf{g}_{\alpha^{\prime} \beta^{\prime}}\left(\tau,{ }^{\prime} u\right) d d^{\prime} \mathbf{e}^{\alpha^{\prime}} \otimes d^{\prime} \mathbf{e}^{\beta^{\prime}}=q_{i}\left(\tau, x^{k}\right) d x^{i} \otimes d x^{i} \\
& +q_{3}\left(\tau, x^{k}, y^{3}\right) \mathbf{e}^{3} \otimes \mathbf{e}^{3}-\left[\breve{N}\left(\tau, x^{k}, y^{3}\right)\right]^{2} \mathbf{e}^{4} \otimes \mathbf{e}^{4}+ \\
& \times{ }^{\prime} q^{a_{2}}\left(\tau, x^{k}, y^{3}, p_{b_{2}}\right)^{\prime} \mathbf{e}_{a_{2}} \otimes \mathbf{e}_{a_{2}} \\
& +q^{7}\left(\tau, x^{k}, y^{3}, p_{b_{2}}, p_{b_{3}}\right) \mathbf{e}_{7} \otimes \mathbf{e}_{7} \\
& -\left[{ }^{\prime} \tilde{N}\left(\tau, x^{k}, y^{3}, p_{b_{2}}, p_{b_{3}}\right)\right]^{2} \mathbf{e}_{8} \otimes \mathbf{e}_{8},
\end{aligned}
$$

where, for instance, ' $e^{\alpha_{s}}$ are $\mathrm{N}$-adapted bases on total space of respective cotangent Lorentz bundles. This ansatz for metrics is a general $\mathrm{N}$-adapted one for a 8 -d phase space metric which can be written as an extension of a couple of 3-d metrics, $q_{i j}=\operatorname{diag}\left(q_{\grave{i}}\right)=\left(q_{i}, q_{3}\right)$ on a hypersurface $\widetilde{\Xi}_{t}$, and ' $q^{\grave{a} \grave{b}}=$ $\operatorname{diag}\left({ }^{\prime} q^{\grave{a}}\right)=\left({ }^{\prime} q^{a_{2}},{ }^{\prime} q^{7}\right)$ on a hypersurface ${ }^{~} \widetilde{\Xi}_{E}$, if

$q_{3}=g_{3}, \breve{N}^{2}=-g_{4}$ and ${ }^{\prime} q^{7}={ }^{\prime} g^{7},{ }^{\prime} \check{N}^{2}=-{ }^{\prime} g^{8}$,

where $\breve{N}$ is the lapse function on the base and ' $\breve{N}$ is the lapse function in the co-fiber (here we note that "the inverse hat" labels are a bit different for the 4-th and 8-th coordinate).
On $T \mathbf{V}$, the nonholonomic lapse and shift variables are introduced in a similar way, which results in d-matric parameterizations

$$
\begin{aligned}
\mathbf{g}= & \mathbf{g}_{\alpha^{\prime} \beta^{\prime}}(\tau, u) d \mathbf{e}^{\alpha^{\prime}} \otimes d \mathbf{e}^{\beta^{\prime}}=q_{i}\left(\tau, x^{k}\right) d x^{i} \otimes d x^{i}+q_{3}\left(\tau, x^{k}, y^{3}\right) \mathbf{e}^{3} \\
& \otimes \mathbf{e}^{3}-\left[\check{N}\left(\tau, x^{k}, y^{3}\right)\right]^{2} \mathbf{e}^{4} \otimes \mathbf{e}^{4}+ \\
& \times q^{a_{2}}\left(\tau, x^{k}, y^{3}, y^{b_{2}}\right) \mathbf{e}_{a_{2}} \otimes \mathbf{e}_{a_{2}}+q^{7}\left(\tau, x^{k}, y^{3}, y^{b_{2}}, y^{b_{3}}\right) \mathbf{e}_{7} \otimes \mathbf{e}_{7} \\
& -\left[\check{N}\left(\tau, x^{k}, y^{3}, y^{b_{2}}, y^{b_{3}}\right)\right]^{2} \mathbf{e}_{8} \otimes \mathbf{e}_{8} .
\end{aligned}
$$

We consider respective hypersurface formulas, $q_{i j}=\operatorname{diag}\left(q_{i}\right)$ $=\left(q_{i}, q_{3}\right)$ on a hypersurface $\widetilde{\Xi}_{t}$, and $q_{\grave{a} \grave{b}}=\operatorname{diag}\left(q_{\grave{a}}\right)=$ $\left(q_{a_{2}}, q_{7}\right)$ on a hypersurface $\widetilde{\Xi}_{E}$, if $q_{3}=g_{3}, \breve{N}^{2}=-g_{4}$ and $q_{7}=g_{7}, \breve{N}^{2}=-g_{8}$, where $\breve{N}$ is the lapse function on the base and ' $\breve{N}$ is the lapse function in the fiber

\subsubsection{Relativistic thermodynamic models for Lagrange-Hamilton geometric evolution}

G. Perelman's very original idea was that the geometric flows of Riemannian metrics can be characterized by an analogous thermodynamic model [1]. In this work, we consider relativistic mechanical generalizations related to geometric flow approaches to classical mechanics $[19,20]$.

\section{Some basic concepts from statistical thermodynamics}

To elaborate analogous thermodynamical models we can consider a partition function $Z=\int \exp (-\beta E) d \omega(E)$ for the canonical ensemble at temperature $\beta^{-1}=T$ (one should not confuse this $T$ for thermodynamics with standard tensor notations with $T$ containing indices for respective for energymomentum tensors and/or torsion in MGTs) being defined by the measure taken to be the density of states $\omega(E)$. The thermodynamical values are computed in standard form for the average energy, $\mathcal{E}=\langle E\rangle:=-\partial \log Z / \partial \beta$, the entropy $\mathcal{S}:=\beta\langle E\rangle+\log Z$ and the fluctuation $\eta:=\left\langle(E-\langle E\rangle)^{2}\right\rangle=$ $\partial^{2} \log Z / \partial \beta^{2}$. Using $Z$, we can define a conventional state density (generalized as a density matrix, it is important for elaborations in geometric flow thermodynamics and information theory, see next sections)

$\sigma(\beta, E)=Z^{-1} e^{-\beta E}$.

Considering $\log \sigma=-\beta \mathcal{E}-\log Z$, we define the relative entropy between any state density $\rho$ and $\sigma$,

$$
\begin{aligned}
\mathcal{S}(\rho \| \sigma): & =\mathcal{S}(\rho)+\int(\beta \mathcal{E}+\log Z) \rho d \omega(E) \\
& =\beta[\mathcal{E}(\rho)-T \mathcal{S}(\rho)]+\log Z,
\end{aligned}
$$

where the average energy computed in the density matrix $\rho$ is $\mathcal{E}(\rho)=\int \mathcal{E} \rho d \omega(E)$. The free energy corresponding to $\rho$ is

$\mathcal{F}(\rho):=\mathcal{E}(\rho)-T \mathcal{S}(\rho)$. 
We note that if $\log Z$ is independent on $\rho$ (as we consider in above formulas) we have $\mathcal{S}(\sigma \| \sigma)=0$. This allows us to write

$\mathcal{S}(\rho \| \sigma)=\beta[\mathcal{F}(\rho)-\mathcal{F}(\sigma)]$.

In this work, we study the geometric flow evolution of thermodynamics systems that preserves the thermal equilibrium at temperature $\beta$ but maps $\rho \rightarrow \rho^{\prime}$ (such density states are different ones) keeping the same density state $\sigma$. We can provide a realistic physical interpretation for such systems if

$\mathcal{S}(\rho \| \sigma) \geq \mathcal{S}\left(\rho^{\prime} \| \sigma\right)$, i.e. $\mathcal{F}(\rho) \geq \mathcal{F}\left(\rho^{\prime}\right)$.

So, we should elaborate on thermodynamic geometric flows that preserve the thermal equilibrium and can only reduce the free energy. These aspects connect mechanical flow models to the second low of thermodynamics. ${ }^{8}$

\section{Thermodynamic values for relativistic Lagrange-Hamilton flows:}

For relativistic geometric flows of mechanical systems, we introduce respective thermodynamic generating functions

$$
\begin{aligned}
\widetilde{\mathcal{Z}}[\widetilde{\mathbf{g}}(\tau)] & =\widetilde{\int} e^{-\tilde{f}} \sqrt{\left|\widetilde{\mathbf{g}}_{\alpha \beta}\right|} d^{8} u(-\tilde{f}+16), \text { for } T \mathbf{V} ; \\
{ }^{\prime} \widetilde{\mathcal{Z}}\left[{ }^{\prime} \widetilde{\mathbf{g}}(\tau)\right] & =\widetilde{\int} e^{-' \widetilde{f}} \sqrt{\left|\widetilde{\mathbf{g}}_{\alpha \beta}\right|} d^{8} ' u(-' \tilde{f}+16), \text { for } T^{*} \mathbf{V},
\end{aligned}
$$

where the respective functional dependence is given by $[\widetilde{\mathbf{g}}(\tau)]$ and $[\tilde{\mathbf{g}}(\tau)]$ (we shall not write such dependencies if that will not result in ambiguities). For a thermodynamic analogous interpretation we can consider that a density state $\sigma$ is associated to $\widetilde{\mathbf{g}}_{\alpha \beta}$, we can write in functional form $\sigma[\widetilde{\mathbf{g}}]$, but the geometric evolution may involve densities $\rho[1, \tilde{\mathbf{g}}]$ and $\rho^{\prime}[1 \widetilde{\mathbf{g}}]$, where the left label 1 is used in order to distinguish two d-metrics $\widetilde{\mathbf{g}}$ and ${ }_{1} \widetilde{\mathbf{g}}$. On cotangent bundles, such values are written respectively ' $\sigma\left[{ }^{\prime} \widetilde{\mathbf{g}}\right],{ }^{\prime} \rho\left[\begin{array}{l}1 \\ 1\end{array}\right.$

Generalizing for nonholonomic deformations of metrics and d-connections respective formulas related to respective

\footnotetext{
8 It should be noted here that G. Perelman treated $\tau=\beta^{-1}$ as a temperature parameter and that he introduced the concept of W-entropy following an analogy to formulas for the entropy in statistical mechanics. We reproduce here the Remark 5.3 and next paragraph, just before section 6 in [1]: "An entropy formula for the Ricci flow in dimension two was found by Chow [C]; there seems to be no relation between his formula and ours. .... The interplay of statistical physics and (pseudo)riemannian geometry occurs in the subject of Black Hole Thermodynamics, developed by Hawking et al. Unfortunately, this subject is beyond my understanding at the moment." It should be also emphasized that G. Perelman had not specified what type of underlying microstates and their energy should be taken in order to explain the geometric flows corresponding to certain thermodynamical and gravity models. In this work, we are interested in geometric mechanics and the classical and quantum information theory developing our approaches elaborated in [19,23-29].
}

entropy like functionals (26), (27) and (28), (29), we can define and compute such relativisitic thermodynamic values for geometric evolution flows of Lagrange mechanical systems,

$$
\begin{aligned}
& \text { average flow energy: } \widetilde{\mathcal{E}}=-\tau^{2} \tilde{\int} e^{-\widetilde{f}} \sqrt{\left|q_{1} q_{2} q_{3} \breve{N} q_{5} q_{6} q_{7} \check{N}\right|} \delta^{8} \\
& \times u\left({ }_{s} \widetilde{R}+|\widetilde{\mathbf{D}} \widetilde{f}|^{2}-\frac{8}{\tau}\right), \\
& \text { flow entropy: } \widetilde{\mathcal{S}}=-\tilde{\int} e^{-\widetilde{f}} \sqrt{\left|q_{1} q_{2} q_{3} \breve{N} q_{5} q_{6} q_{7} \check{N}\right|} \delta^{8} u \\
& \times\left[\tau\left({ }_{s} \widetilde{R}+|\widetilde{\mathbf{D}} \tilde{f}|^{2}\right)+\tilde{f}-16\right], \\
& \text { flow fluctuation: } \widetilde{\eta}=-\tilde{\int} e^{-\widetilde{f}} \sqrt{\left|q_{1} q_{2} q_{3} \breve{N} q_{5} q_{6} q_{7} \check{N}\right|} \delta^{8} \\
& \times u\left[\left|\widetilde{\mathbf{R}}_{\alpha \beta}+\widetilde{\mathbf{D}}_{\alpha} \widetilde{\mathbf{D}}_{\beta} \tilde{f}-\frac{1}{2 \tau} \mathbf{g}_{\alpha \beta}\right|^{2}\right],
\end{aligned}
$$

where $\delta^{8} u$ contains $\mathrm{N}$-elongated differentials of type (8) (when we compute such integrals in $\mathrm{N}$-adapted form). Using such values, we can compute the respective free energy (32) and relative entropy (33),

$$
\begin{aligned}
\widetilde{\mathcal{F}}\left({ }_{1} \widetilde{\mathbf{g}}\right)= & \widetilde{\mathcal{E}}\left({ }_{1} \widetilde{\mathbf{g}}\right)-\beta^{-1} \widetilde{\mathcal{S}}\left({ }_{1} \widetilde{\mathbf{g}}\right) \text { and } \widetilde{\mathcal{S}}\left({ }_{1} \widetilde{\mathbf{g}} \| \sigma\right) \\
& =\beta\left[\widetilde{\mathcal{F}}\left({ }_{1} \widetilde{\mathbf{g}}\right)-\widetilde{\mathcal{F}}(\widetilde{\mathbf{g}})\right], \text { where } \\
\widetilde{\mathcal{E}}\left({ }_{1} \widetilde{\mathbf{g}}\right)= & -\tau^{2} \widetilde{\int} e^{-\widetilde{f}} \sqrt{\left|q_{1} q_{2} q_{3} \breve{N} q_{5} q_{6} q_{7} \check{N}\right|} \delta^{8} \\
& \times u\left[{ }_{s} \widetilde{R}\left({ }_{1} \widetilde{\mathbf{g}}\right)+\left|\widetilde{\mathbf{D}}\left({ }_{1} \widetilde{\mathbf{g}}\right) \tilde{f}(\tau, u)\right|^{2}-\frac{8}{\tau}\right], \\
\widetilde{\mathcal{S}}\left({ }_{1} \widetilde{\mathbf{g}}\right)= & -\widetilde{\int} e^{-\widetilde{f}} \sqrt{\left|q_{1} q_{2} q_{3} \breve{N} q_{5} q_{6} q_{7} \check{N}\right|} \delta^{8} u \\
& \times\left[\tau\left({ }_{s} \widetilde{R}\left({ }_{1} \widetilde{\mathbf{g}}\right)+\left|\widetilde{\mathbf{D}}\left({ }_{1} \widetilde{\mathbf{g}}\right) \widetilde{f}(\tau, u)\right|^{2}\right)+\tilde{f}(\tau, u)-16\right] .
\end{aligned}
$$

Such values are in relativistic thermodynamic relation if the second thermodynamic law (34) is satisfied. This impose certain constraints on the class of normalizing and generating functions we consider for the termodynamic description of such relativistic Lagrange systems.

For geometric evolution flows of Hamilton mechanical systems, the relativistic thermodynamic values are

$$
\begin{aligned}
& { }^{\prime} \tilde{\mathcal{E}}=-\tau^{2} \cdot \tilde{\int} e^{-\mid \tilde{f}} \sqrt{\left|q_{1} q_{2} q_{3} \breve{N}{ }^{\prime} q_{5}{ }^{\prime} q_{6}{ }^{\prime} q_{7}{ }^{\prime} \check{N}\right|} \delta^{8} \text {, } \\
& \times u\left({ }_{s} \widetilde{R}+\left|\widetilde{\mathbf{D}}^{\prime} \widetilde{f}\right|^{2}-\frac{8}{\tau}\right), \\
& \text { ' } \widetilde{\mathcal{S}}=-\tilde{\int} e^{-\mid \tilde{f}} \sqrt{\left|q_{1} q_{2} q_{3} \breve{N}^{\prime} q_{5}{ }^{\prime} q_{6}{ }^{\prime} q_{7}{ }^{\prime} \check{N}\right|} \delta^{8} \\
& \times u\left[\tau\left({ }_{s}{ }_{s} \widetilde{R}+\left|{ }^{\prime} \widetilde{\mathbf{D}}^{\prime} \widetilde{f}\right|^{2}\right)+{ }^{\prime} \tilde{f}-16\right],
\end{aligned}
$$




$$
\begin{aligned}
{ }^{\prime} \tilde{\eta}= & -{ }^{\prime} \tilde{\int} e^{-' \tilde{f}} \sqrt{\left|q_{1} q_{2} q_{3} \breve{N}^{\prime} q_{5}{ }^{\prime} q_{6}{ }^{\prime} q_{7}{ }^{\prime} \check{N}\right|} \delta^{8}, \\
& \times u\left[\left|{ }^{\prime} \widetilde{\mathbf{R}}_{\alpha \beta}+{ }^{\prime} \widetilde{\mathbf{D}}_{\alpha}{ }^{\prime} \widetilde{\mathbf{D}}_{\beta}{ }^{\prime} \tilde{f}-\frac{1}{2 \tau}{ }^{\prime} \mathbf{g}_{\alpha \beta}\right|^{2}\right] .
\end{aligned}
$$

Other thermodynamic values and conditions can be computed by analogy to above relativistic Lagrange thermodynamic configurations and formulas (32), (33) and (34).

Finally we note that above formulas can be written respectively and equivalently in terms of the canonical dconnections $\widehat{\mathbf{D}}$ and,$\widehat{\mathbf{D}}$ if we consider nonholonomic deformations to certain systems of nonlinear partial differential equations with general decoupling.

\subsubsection{Curved spaces emerging from relativistic phase space geometric evolution}

The geometric flow evolution of 4-d (pseudo) Riemannian configurations is described by nonholonomically modified Perelman's functionals integrated on (co) fiber variables (26), (27) and/or (28), (29). A subclass of such relativistic flows are generated for parameterizations with d-metrics (1) and (2)). Re-defining the normalizing functions, $\widetilde{f} \rightarrow$ $\widehat{f}\left(x^{1}, x^{2}, y^{3}, y^{4}\right)$ and/or $\widetilde{f} \rightarrow \mid \widehat{f}$, for general frame transforms on a base Lorentz manifold, we obtain such functionals:

$$
\begin{aligned}
\widehat{\mathcal{F}} & =\int_{t_{1}}^{t_{2}} \int_{\widehat{\Xi}_{t}} e^{-\widehat{f}} \sqrt{\left|q_{1} q_{2} q_{3} \breve{N}\right|} \delta^{4} u\left({ }_{s} \widehat{R}+|\widehat{\mathbf{D}} \widehat{f}|^{2}\right) \text { and } \\
\widehat{\mathcal{W}}= & \int_{t_{1}}^{t_{2}} \int_{\widehat{\Xi}_{t}}(4 \pi \tau)^{-4} e^{-f} \sqrt{\left|q_{1} q_{2} q_{3} \breve{N}\right|} \delta^{4} u\left[\tau \left({ }_{s} \widehat{R}+\mid{ }_{h} \widehat{\mathbf{D}} \widehat{f}\right.\right. \\
& \left.\left.\quad+\left|+{ }_{v} \widehat{\mathbf{D}} \widehat{f}\right|\right)^{2}+\widehat{f}-8\right] .
\end{aligned}
$$

In these formulas, geometric fllows of ${ }_{s} \widehat{R}$ are for respective $\widehat{\mathbf{D}}=\left({ }_{h} \widehat{\mathbf{D}},{ }_{v} \widehat{\mathbf{D}}\right)$ on a family of bases $\mathbf{V}(\tau)$, where the normalizing function $\widehat{f}(\tau, u)$ satisfies the conditions $\int_{t_{1}}^{t_{2}} \int_{\widetilde{\Xi}_{t}} \widehat{\mu} \sqrt{\left|q_{1} q_{2} q_{3} \breve{N}\right|} \delta^{4} u=1$ for $\widehat{\mu}=(4 \pi \tau)^{-4} e^{-\widehat{f}}$, when the coefficient $8=2 \times 4$ is taken for 4-d manifolds.

Using formulas for distortions of connections (25) redefined for 4-d nonholonomic manifolds, the functionals (38) can re-written using geometric data $(\widetilde{\mathbf{g}}, \widetilde{\mathbf{D}})$ and/or $(\mathbf{g}, \nabla)$. Such F- and W-functionals define nonholonomic geometric evolution flows of vacuum gravitational fields in MGTs and GR, see details in Refs. [25-28]. We can consider that, in principle, (modified) gravitational interactions are induced as certain emergent fields from geometric evolution flows of mechanical Lagrange/ Hamilton systems.

The thermodynamic generating function corresponding to (38) can be defined in the form

$$
\widehat{\mathcal{Z}}=\int_{t_{1}}^{t_{2}} \int_{\widehat{\Xi}_{t}} e^{-\widehat{f}} \sqrt{\left|q_{1} q_{2} q_{3} \breve{N}\right|} \delta^{4} u(-\widehat{f}+8), \text { for } \mathbf{V} .
$$

In result, we can characterize emergent (pseudo) Riemannian geometries by such relativistic thermodynamic values,

$$
\begin{aligned}
\widehat{\mathcal{E}}= & -\tau^{2} \int_{t_{1}}^{t_{2}} \int_{\widehat{\Xi}_{t}} e^{-\widehat{f}} \sqrt{\mid q_{1} q_{2} q_{3} \breve{N}} \mid \delta^{4} u\left({ }_{s} \widehat{R}+|\widehat{\mathbf{D}} \widehat{f}|^{2}-\frac{4}{\tau}\right), \\
\widehat{\mathcal{S}}= & -\int_{t_{1}}^{t_{2}} \int_{\widehat{\Xi}_{t}} e^{-\widehat{f}} \sqrt{\left|q_{1} q_{2} q_{3} \breve{N}\right|} \delta^{4} u \\
& \times\left[\tau\left({ }_{s} \widehat{R}+|\widehat{\mathbf{D}} \widehat{f}|^{2}\right)+\widehat{f}-8\right], \\
\widehat{\eta}= & -\int_{t_{1}}^{t_{2}} \int_{\widehat{\Xi}_{t}} e^{-\widehat{f} \sqrt{\left|q_{1} q_{2} q_{3} \breve{N}\right|} \delta^{4} u\left[\mid \widehat{\mathbf{R}}_{\alpha \beta}+\widehat{\mathbf{D}}_{\alpha} \widehat{\mathbf{D}}_{\beta} \tilde{f}\right.} \\
& \left.-\left.\frac{1}{2 \tau} \mathbf{g}_{\alpha \beta}\right|^{2}\right],
\end{aligned}
$$

where all geometric objects and indices are for 4-d base manifolds. Up to nonholonomic frame transforms and deformations of connections, such vaules encode explicit information (integrated on fiber variables and/or projected on base spacetime manifolds) on certain total space Lagrange/ Hamilton generating functions.

There are different approaches for elaborating models of 3-d Ricci flow evolution of mechanical systems and (emergent of prescribe) 4-d spacetimes with pseudo-Euclidean signature. In principle, there are two general possibilities. In the first case, is to approach the problem as in the theories of stochastic / diffusion and kinetic processes with local anisotropy, fractional geometric evolution etc. For such models, one elaborates on thermofield models of Ricci flow evolution on imaginary time $\tau=-i t(0 \leq \tau \leq 1 / \kappa T$, where $\kappa$ is Boltzmann's constant. In corresponding formulas, the symbol $T$ is used for the temperature (such a letter with respective indices for torsion and energy-momentum tensors is also used in gravity theories). In result, the pseudo-Riemannian spacetime is transformed into a Riemannian configuration space as one elaborates in thermal and/or finite temperature quantum field theory. The second class consists from theories modelled on 3-d hypersurfaces and evolving relativistically, for instance, on a 4-d Ricci soliton configuration. In such cases, the evolution parameter $\tau \sim t$ is a time like coordinate. In this work, we study evolution of relativistic mechanics systems on a temperature like parameter $\tau \sim T$.

\subsubsection{Effective nonholonomic 3-d space like hypersurface $F$ - and W-functionals}

Lagrange and Hamilton mechanical systems on Lorentz manifolds can be also characterized by 3-d space like hypersurface functionals. Such values can be defined respectively for (38) and (39) for any 3+1 splitting with 3-d closed hypersurface fibrations $\widehat{\Xi}_{t}$.

We denote by ${ }_{0} \widehat{\mathbf{D}}=\widehat{\mathbf{D}}_{\mid \widehat{\Xi}_{t}}$ the canonical d-connection $\widehat{\mathbf{D}}$ defined on a 3-d hypersurface $\widehat{\mathbf{\Xi}}_{t}$. In a similar form, there are defined hypersurface "tilde" variables with ${ }_{\circ} \widetilde{\mathbf{D}}=\widetilde{\mathbf{D}}_{\mid \widetilde{\Xi}_{t}}$ determined as a projection of 8-d canonical Lagrange-Hamilton 
d-connection defined on a 3-d hypersurface $\widetilde{\Xi}_{t}$. For geometric flow evolution, all such values depend on a temperature like parameter $\tau\left(\tau^{\prime}\right)$ with possible scale re-definitions for another parameter $\tau^{\prime}$ etc. We define also ${ }_{0}^{s} \widehat{R}:={ }^{s} \widehat{R}_{\mid \widehat{\Xi}_{t}}$ and ${ }_{0}^{s} \widetilde{R}:={ }^{s} \widetilde{R}_{\mid \widetilde{\Xi}_{t}}$. Using $q_{i}(\tau)=\left[q_{i}(\tau), q_{3}(\tau)\right]$ in a family of $\mathrm{d}$-metrics (see, for instance, (31 )), we define 3-d F- and Wfunctionals parameterized in $\mathrm{N}$-adapted form for the canonical d-connection,

$$
\begin{aligned}
{ }_{\circ} \widehat{\mathcal{F}}= & \int_{\widehat{\Xi}_{t}} e^{-} \widehat{\circ} \sqrt{\mid q_{1} q_{2} q_{3}} \mid \delta \dot{x}^{3}\left[\left({ }_{\circ}^{s} \widehat{R}+\left|{ }_{\circ} \widehat{\mathbf{D}} \circ \widehat{\circ}\right|^{2}\right)\right], \text { and } \\
{ }_{\circ} \widehat{\mathcal{W}}= & \int_{\widehat{\Xi}_{t}} \widehat{o} \widehat{\mu} \sqrt{\left|q_{1} q_{2} q_{3}\right|} \delta \dot{x}^{3}\left[\tau\left({ }_{\circ}^{s} \widehat{R}+\left|{ }_{\circ}^{h} \widehat{\mathbf{D}} \circ \widehat{f}\right|+\left|{ }_{\circ}^{v} \widehat{\mathbf{D}} \circ \widehat{f}\right|\right)^{2}\right. \\
& \left.+{ }_{\circ} \widehat{f}-6\right] .
\end{aligned}
$$

These functionals are for a redefined normalization function $\circ \widehat{f}$. We can always chose a necessary type scaling function $\circ \widehat{f}$ which satisfies normalization conditions $\int_{\widehat{\Xi}_{t}} \circ \widehat{\mu} \sqrt{\left|q_{1} q_{2} q_{3}\right|} \delta \dot{x}^{3}=1$ for $\circ \widehat{\mu}=(4 \pi \tau)^{-3} e^{-\circ \widehat{f}}$. For topological considerations, the type of normalization is not important. Such conditions can be imposed as via frame/coordinate transforms and deformations of linear connections which allows to solve derived geometric flow evolution equations in explicit form. For certain applications, we can consider $\circ \widehat{f}$ as an undetermined scalar function which can be related to certain conformal transforms or reparameterizations.

Using ${ }_{\circ} \widehat{\mathcal{F}}(40)$ and the thermodynamic generating function $。 \widehat{\mathcal{Z}}=\exp \left[\int_{\widehat{\Xi}_{t}} \circ \widehat{\mu} \sqrt{\left|q_{1} q_{2} q_{3}\right|} \delta \dot{x}^{3}(-\circ \widehat{f}+6)\right]$, we can define and compute such 3-d hypersurface thermodynamic values:

$$
\begin{aligned}
& { }_{\circ} \widehat{\mathcal{E}}=-\tau^{2} \int_{\widehat{\Xi}_{t}}{ }_{\circ} \widehat{\mu} \sqrt{\left|q_{1} q_{2} q_{3}\right|} \delta \dot{x}^{3}\left({ }_{\circ}^{s} \widehat{R}+\left|{ }_{\circ} \widehat{\mathbf{D}} \circ \widehat{\circ}\right|^{2}-\frac{3}{\tau}\right), \\
& \widehat{\circ} \widehat{\mathcal{S}}=-\int_{\widehat{\Xi}_{t}}{ }_{0} \widehat{\mu} \sqrt{\left|q_{1} q_{2} q_{3}\right|} \delta \dot{x}^{3} \\
& \times\left[\tau\left({ }_{0}^{s} \widehat{R}+\left|{ }_{\circ} \widehat{\mathbf{D}} 。 \widehat{f}\right|^{2}\right)+\tilde{f}-6\right], \\
& \widehat{\circ}=2 \tau^{4} \int_{\widehat{\Xi}_{t} \circ} \widehat{\mu} \sqrt{\left|q_{1} q_{2} q_{3}\right|} \delta \dot{x}^{3}\left[\mid{ }_{\circ} \widehat{\mathbf{R}}_{i j}+{ }_{\circ} \widehat{\mathbf{D}}_{i}{ }_{\circ} \widehat{\mathbf{D}}_{j} \tilde{f}\right. \\
& \left.-\left.\frac{1}{2 \tau} q_{i j}\right|^{2}\right] \text {. }
\end{aligned}
$$

These formulas can be considered for 4-d configurations (39) taking the lapse function $\breve{N}=1$ for N-adapted Gaussian coordinates. We can also write such formulas in equivalent form using geometric data $(\widetilde{\mathbf{q}}, \quad \widetilde{\mathbf{D}})$ and/or $(\mathbf{q}, \circ \nabla)$ for respectively re-defined normalizing functions. For LCconfigurations, the 3-d hypersurface formulas (40), (41) and (42) transform into the standard ones from G. Perelman's preprint [1]. The main difference is that in our approach such Riemannian hypersufrace flow evolution scenarios are determined by Lagrange-Hamilton mechanical systems.
3.2 Generalized R. Hamilton flow evolution equations and geometric mechanics

In this section we show that Lagrange and/or Hamilton mechanical systems are characterized not only by dynamical equations (which is well-known from classical mechanics [16-18]) but also by certain classes of geometric flow evolution equations $[19,20]$. Relativistic variants of such systems of nonlinear PDEs can be proven by applying a variational $\mathrm{N}$-adapted calculus for respective F- and W-functionals as in [19,23-29]. For holonomic Riemannian manifolds, such proofs can be found in $[1,13-15]$.

\subsubsection{Riemannian geometric flows on 3-d spacelike hypersurface}

Applying a $\mathrm{N}$-adapted variational procedure on a 3-d hypersurface to a functional (40) or (41) defined by data $\left(\widetilde{g}_{i \bar{j}}, \widetilde{\nabla}\right)$, we obtain such equations in the form

$\frac{\partial \widetilde{g}_{i \grave{j}}}{\partial \tau}=-2 \widetilde{R}_{i \grave{j}}$,

where $\tau$ is an evolution real parameter. There are used local coordinates $u^{i}$ with indices $\grave{i}, \grave{j}=1,2,3$ and Ricci tensor $\widetilde{R}_{i j}$ for a 3-d Riemannian manifold (in this work constructed as an emergent from geometric mechanics curve space). These equations are equivalent to the (non-relativistic) Ricci flow evolution equations postulated heuristically by $\mathrm{R}$. Hamilton [10-12]. G. Perelman proved such equations using his $\mathrm{F}$ - and $\mathrm{W}$-functionals.

The equations (43) describe a nonlinear diffusion process for geometric flow evolution of relativistic mechanical systems encoded up to frame transforms into 3-d Riemannian metrics (we can omit tilde and write $g_{i j}$ in certain general covariant form). For models with small deformations of a 3-d

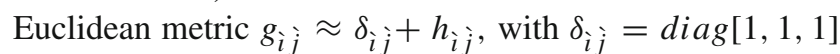

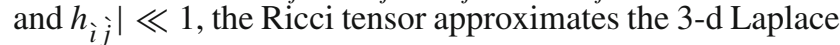
operator $\Delta=\frac{\partial^{2}}{\left(\partial u^{1}\right)^{2}}+\frac{\partial^{2}}{\left(\partial u^{2}\right)^{2}}+\frac{\partial^{2}}{\left(\partial u^{3}\right)^{2}}$. On 3-d hypersurfaces and "slow" evolution, the geometric flows of mechanical systems are described by a linear diffusion equation with $R_{\grave{i} j} \sim \Delta h_{\grave{i} j}$. For relativistic models, we have to elaborate on hydrodynamic anisotropic like transports of entropic fields and derived geometric objects [24-26].

\subsubsection{Geometric flow equations for relativistic Lagrange-Hamilton systems}

Applying a $\mathrm{N}$-adapted variational procedure with a corresponding re-definition of normalizing function for $\widetilde{\mathcal{F}}(26)$ determined by geometric data $\left(\widetilde{\mathbf{g}}=\left\{\widetilde{\mathbf{g}}_{\mu \nu}=\left[\widetilde{g}_{i j}, \widetilde{g}_{a b}\right]\right\}\right.$, $\left.\widetilde{\mathbf{N}}=\left\{\widetilde{N}_{i}^{a}\right\}, \widetilde{\mathbf{D}}\right)$, we obtain a system of nonlinear PDEs generalizing the R. Hamilton equations for geometric flow evolu- 
tion of relativistic Lagrange systems,

$$
\begin{aligned}
\partial_{\tau} \widetilde{g}_{i j} & =-2 \widetilde{\mathbf{R}}_{i j} ; \partial_{\tau} \widetilde{g}_{a b}=-2 \widetilde{\mathbf{R}}_{a b} ; \\
\widetilde{\mathbf{R}}_{i a} & =\widetilde{\mathbf{R}}_{a i}=0 ; \widetilde{\mathbf{R}}_{i j}=\widetilde{\mathbf{R}}_{j i} ; \widetilde{\mathbf{R}}_{a b}=\widetilde{\mathbf{R}}_{b a} ; \\
\partial_{\tau} \widetilde{f} & \left.=-\widetilde{\square} \tilde{f}+|\widetilde{\mathbf{D}} \widetilde{f}|^{2}-{ }_{s} \widetilde{R}\right) .
\end{aligned}
$$

In these formulas, $\widetilde{\square}(\tau)=\widetilde{\mathbf{D}}^{\alpha}(\tau) \widetilde{\mathbf{D}}_{\alpha}(\tau)$ and the conditions $\widetilde{\mathbf{R}}_{i a}=0$ and $\widetilde{\mathbf{R}}_{a i}=0$ for the Ricci tensor $\operatorname{Ric}[\widetilde{\mathbf{D}}]=\left\{\widetilde{\mathbf{R}}_{\alpha \beta}=\right.$ $\left.\left[\widetilde{\mathbf{R}}_{i j}, \widetilde{\mathbf{R}}_{i a}, \widetilde{\mathbf{R}}_{a i}, \widetilde{\mathbf{R}}_{a b}\right]\right\}$ are imposed in order to keep a symmetric metric evolution.

For the geometric flow evolution of relativisitic Hamilton mechanical systems, the analogs of (44) can be written (in principle, such equations can be proven in abstract form dualizing geometric objects from the tangent Lorentz bundles to respective cotangent bundles and functional $' \widetilde{\mathcal{F}}(28))$ for the geometric data $\left({ }^{\widetilde{\mathbf{g}}}=\left\{\widetilde{\mathbf{g}}_{\mu \nu}=\right.\right.$ $\left.\left.\left[{ }^{\prime} \widetilde{g}_{i j}, ' \widetilde{g}_{a b}\right]\right\}, ' \widetilde{\mathbf{N}}=\left\{{ }^{\prime} \widetilde{N}_{i}^{a}\right\}, ' \widetilde{\mathbf{D}}\right)$,

$$
\begin{aligned}
& \partial_{\tau}{ }^{\prime} \tilde{g}_{i j}=-2{ }^{\prime} \widetilde{\mathbf{R}}_{i j} ; \partial_{\tau}{ }^{\prime} \widetilde{g}_{a b}=-2{ }^{\prime} \widetilde{\mathbf{R}}_{a b} ; \\
& { }^{\prime} \widetilde{\mathbf{R}}_{i a}={ }^{\prime} \widetilde{\mathbf{R}}_{a i}=0 ;{ }^{\prime} \widetilde{\mathbf{R}}_{i j}={ }^{\prime} \widetilde{\mathbf{R}}_{j i} ;{ }^{\prime} \widetilde{\mathbf{R}}_{a b}={ }^{\prime} \widetilde{\mathbf{R}}_{b a} ; \\
& \left.\partial_{\tau}{ }^{\prime} \widetilde{f}=-{ }^{\prime} \widetilde{\square} \widetilde{f}+\left|{ }^{\prime} \widetilde{\mathbf{D}}^{\prime} \widetilde{f}\right|^{2}-{ }_{s}{ }_{s} \widetilde{R}\right), \\
& \text { where }{ }^{\prime} \widetilde{\square}(\tau)={ }^{\prime} \widetilde{\mathbf{D}}^{\alpha}(\tau){ }^{\prime} \widetilde{\mathbf{D}}_{\alpha}(\tau) .
\end{aligned}
$$

Using nonholonomic deformations of d-connections (25), respective frame transforms and re-definition of normalizing functions, the geometric flow evolution equations can be written in "hat" variables or for LC-configurations. Imposing corresponding classes of nonholonomic constraints, we may drive the flows of geometric objects in a "pure" mechanical form, or mix the frames and indices and generate new classes of nonholonomic phase spacetimes.

\subsubsection{Nonholonomic Ricci solitons, emergent gravity, and geometric mechanics}

For self-similar configurations in a fixed point $\tau=\tau_{0}$, the geometric flows (43) are described by nonholonomic Ricci soliton equations

$\widetilde{R}_{\grave{i} j}-\lambda \widetilde{g}_{\grave{i} j}=\widetilde{\nabla}_{i} v_{j}+\widetilde{\nabla}_{\grave{j}} v_{\grave{i}}$,

for $\lambda= \pm 1,0$ and a vector field $v_{\grave{j}}$. In these formulas, $\lambda$ is taken for a corresponding normalization function, which defines a 3-d hypersurface version of the Einstein equations with cosmological constant. We keep tilde on symbols in order to emphasize that the geometric objects are determined by certain Lagrange or Hamilton generating function on a 8-d (co) tangent bundle.

In a similar form, we can consider self-similar point $\tau=\tau_{0}$ configurations for the systems of nonlinear PDEs (44) and/or ( 45), when $\partial_{\tau} \widetilde{\mathbf{g}}_{\mu \nu}=0$ and/or $\partial_{\tau} \widetilde{\mathbf{g}}_{\mu \nu}=0$, with a corresponding choice of the normalizing geometric flow functions (for simplicity, we can take a zero vector field $v_{\alpha}=0$ ), the equations (44) transform into relativistic nonholonomic Ricci soliton equations

$$
\begin{aligned}
\widetilde{\mathbf{R}}_{i j} & =\lambda \widetilde{g}_{i \grave{j}}, \widetilde{\mathbf{R}}_{a b}=\lambda \widetilde{g}_{a b}, \widetilde{\mathbf{R}}_{i a}=\widetilde{\mathbf{R}}_{a i}=0, \text { on } T \mathbf{V} ; \\
{ }^{\prime} \widetilde{\mathbf{R}}_{i j} & =\lambda^{\prime} \widetilde{g}_{i j}, \quad \widetilde{\mathbf{R}}_{a b}=\lambda^{\prime} \widetilde{g}_{a b}, \quad{ }^{\prime} \widetilde{\mathbf{R}}_{i a}={ }^{\prime} \widetilde{\mathbf{R}}_{a i}=0, \text { on } T^{*} \mathbf{V} .
\end{aligned}
$$

Such equation can be written in hat and/or LC-variables using nonholonomic deformations of d-connections (25) and frame transforms. Projecting (47) on a base 4-d Lorentz manifold $\mathbf{V}$, we obtain nonholonomically deformed vacuum Einstein equations with cosmological constant $\lambda$.

In this work, we do not study gravitational and matter field geometric field interactions. Nevertheless, we note that in our nonholonomic geometric flow approach to investigating the evolution of Lagrange-Hamilton systems, the gravitational field equations emerge from geometric flows of mechanical systems being characterized by a W-entropy (38) and respective thermodynamical values (39). The gravitational constant can be introduced for identifications with respective spherical symmetric solutions with an additional assumption that at long distances the standard Newton gravitational potential is generated. In certain sense, for such theories, a W-entropy acts as an entropic force for the E. Verlinde model [51,52], see proofs in [27-29].

\section{Classical and quantum mechanical geometric information flow theories}

This section is a short introduction to basic aspects of classical and quantum geometric information flow (respectively, GIF and QGIF) models and related subjects from the theory of geometric evolution of relativistic mechanical systems (elaborated in previous sections). Using modified G. Perelman entropy functionals and the nonholonomically adapted von Neumann entropy for quantum density matrices, there are defined quantum conditional entropy, relative entropy, and mutual information values as basic ingredients of the QGIF theory.

4.1 Geometric information flow theory of classical mechanical systems

Classical information theory is based on fundamental concepts of Shannon, conditional and relative entropies [37,38, 47-49]. To elaborate on classical aspects of geometric information flow, GIF, models we have to define analogous values determined by (modified) Perelman entropy functionals and associated thermodynamical models.

\subsubsection{Shannon entropy and geometric flow entropy in information theories}

Let us remember the general definition of the Shannon entropy $S_{B}$ of a probability distribution for a random variable 
$B$ taking certain values $b_{1}, b_{2}, \ldots, b_{k}$ (for instance, to send a long message $N \gg 1$ with $k$ letters) with respective probabilities to observe such values $p_{\underline{1}}, p_{\underline{2}}, \ldots, p_{\underline{k}} .{ }^{9}$ By definition,

$S_{B}:=-\sum_{\underline{j}=1}^{\underline{k}} p_{\underline{j}} \log p_{\underline{j}} \geq 0$ with $\sum_{\underline{j}=1}^{\underline{k}} p_{\underline{j}}=1$.

This is for the probability theory with random variables. In classical information models, $\underline{N} S_{B}$ is the number of bits of information which can be extracted from a message with $N$ symbols which are randomly generated. For engineering applications, $N S_{B}$ is the number of bits to which a message with $N$ letters can be compressed. Typically, such messages are not randomly generated but contain certain information. To encode certain real messages with correlations between letters (for instance, words for grammar and syntax) and loose less modifications is a more complex random process. In the ideal gaze limit (ignoring correlations), we can consider that the entropy of a long message is just $N S$, when $S$ is the entropy of a message consisting of only one letter. We can formalize the constructions as models of statistical mechanics if we introduce a classical Hamiltonian $H$ determining the probability of a $i$-th symbol $b_{i}$ in a statistical thermodynamical model via formula $p_{i}=2^{-H\left(b_{i}\right)}$.

The theory of geometric flows is different from the standard theory of random processes, classical information models and "simple" engineering applications. The flow evolution is characterized by the W-entropy and (which is important for our further developments) additional assumptions on associated statistical thermodynamic values like mean energy, entropy and fluctuation. For classical mechanical systems, such values are canonically determined by generating functions $\widetilde{L}$ and $\widetilde{H}$, see formulas $\widetilde{\mathcal{W}}(27)$ and ' $\widetilde{\mathcal{W}}(29)$, and, respectively, for flow evolution of Hessian metrics, by $[\widetilde{\mathcal{E}}, \widetilde{\mathcal{S}}, \widetilde{\eta}](36)$ and $\left[\right.$ ' $\left.\widetilde{\mathcal{E}}, ' \widetilde{\mathcal{S}},{ }^{\prime} \tilde{\eta}\right](37)$. On a discrete network with random variables, we can introduce probabilities, for instance, $\tilde{p}_{n}=2^{-\widetilde{H}\left(b_{\underline{n}}\right)}$ and ' $\tilde{p}_{\underline{n}}=2^{-{ }^{\prime} \widetilde{H}\left(b_{\underline{n}}\right)}$, or, for statistical ansambles, $\widetilde{p}_{\underline{n}}=2^{-\widetilde{\mathcal{E}}\left(b_{\underline{n}}\right)}$ and ' $\widetilde{p}_{\underline{n}}=2^{-'{ }^{\prime} \widetilde{\mathcal{E}}\left(b_{\underline{n}}\right)}$. In result, it is possible to elaborate classical information theories determined by effective Hamiltonians $\widetilde{H}$, or energy functionals $\widetilde{\mathcal{E}}$ and $' \widetilde{\mathcal{E}}$. This is for certain discrete versions with probability models and correlations encoding information on geometric flows of mechanical systems.

\footnotetext{
9 In this section, we should not confuse symbols for probabilities $p_{i}$ with similar notations for cofiber coordinates; and a number $N$ is different from the symbol $\mathbf{N}$ used for the $\mathrm{N}$-connections. Here we note that it is almost impossible and not optimal to elaborate an unified system of notations with completely different symbols in an article involving different directions in differential geometry, geometric mechanics, probability and diffusion, classical and quantum information theory. We try to keep traditional notations for different directions in mathematics or physics but (if necessary) underly symbols and provide respective remarks allowing to avoid notation ambiguities.
}

In this section, we elaborate on continuous information flow models encoding geometric evolution of mechanical systems using the thermodynamic entropies $\widetilde{\mathcal{S}}[\widetilde{\mathbf{g}}(\tau)]$ and ${ }^{\prime} \widetilde{\mathcal{S}}\left[{ }^{\prime} \widetilde{\mathbf{g}}(\tau)\right]$ without involving in the constructions probability distributions which appear for random variables. Geometric flows can be described by $\widetilde{\mathcal{S}}[\widetilde{\mathbf{g}}(\tau)]$ and $\quad \widetilde{\mathcal{S}}\left[{ }^{\prime} \widetilde{\mathbf{g}}(\tau)\right]$. We can elaborate equivalent constructions for W-entropies $\mathcal{W}[\widetilde{\mathbf{g}}(\tau)]$ and ' $\left.\widetilde{\mathcal{W}}\left[{ }^{\prime} \widetilde{\mathbf{g}}(\tau)\right]\right)$. Systems under geometric flows are denoted as $\widetilde{B}=\widetilde{B}[\widetilde{\mathbf{g}}(\tau)]$ and ${ }^{\prime} \widetilde{B}={ }^{\prime} \widetilde{B}\left[{ }^{1} \widetilde{\mathbf{g}}(\tau)\right]$ determined by corresponding canonical d-metrics on phase spacetimes.

\subsubsection{Conditional entropy and geometric information flows GIF}

In information theory, there are studied various conventional models with communicating humans called, for instance, Alice and Bob, see $[37,38]$. Let us suppose that Alice sends a message via a noisy telephone connection with many letters (any letter is a random variable $X$ taking possible values $\left.x_{1}, \ldots, x_{k}\right)$. Bob receives instead of $X$ a random variable $\bar{Y}$ consisting from possible letters $y_{1}, \ldots, y_{r}$. In classical information theory, one computes how many bits of information does Bob receives form Alice's message with $N$ letters? Traditionally, the random variables are denoted as $X, Y, Z$ etc. For one variable, the probability to observe $X=x_{i}$ is denoted $P_{X}\left(x_{i}\right)$ subjected to the condition that $\sum_{i} P_{X}\left(x_{i}\right)=1$. The communication between Alice and $\mathrm{Bob}$ is a random process of two variables defined by a joint distribution $P_{X, Y}\left(x_{i}, y_{j}\right)$ as the probability that Alice sends $X=x_{i}$ and Bob hears $\bar{Y}=y_{j}$. It is considered that the value $P_{Y}\left(y_{j}\right)=\sum_{i} P_{X, Y}\left(x_{\underline{i}}, y_{j}\right)$ is the probability that Bob hears $Y=\bar{y}_{j}$ (summation is over all choices of what Alice could send). The conditional probability

$P_{X \mid Y}\left(x_{\underline{i}} \mid y_{\underline{j}}\right):=\frac{P_{X, Y}\left(x_{\underline{i}}, y_{\underline{j}}\right)}{P_{Y}\left(y_{\underline{j}}\right)}$

is by definition a value characterizing that if Bob hear $Y=$ $y_{\underline{j}}$, he can estimate the probability that Alice sent $x_{i}$. We can write for Alice's messages $P_{X}\left(x_{\underline{i}}\right)=\sum_{j} P_{X, Y}\left(x_{\underline{i}}, y_{j}\right)$, or consider $P_{X}\left(x_{i}\right)$ as an independent probability density. Using these formulas, one defines such important values:

$$
\begin{aligned}
S_{X \mid Y=y_{j}}:= & -\sum_{\underline{i}} P_{X \mid Y}\left(x_{\underline{\underline{i}}} \mid y_{\underline{j}}\right) \log P_{X \mid Y}\left(x_{\underline{i}} \mid y_{\underline{j}}\right), \\
& \text { the Shannon entropy of the conditional probability; } \\
S_{X Y}:= & -\sum_{\underline{i}, \underline{j}} P_{X, Y}\left(x_{\underline{i}}, y_{\underline{j}}\right) \log P_{X, Y}\left(x_{\underline{i}}, y_{\underline{j}}\right), \\
& \text { the entropy of joint distribution ; } \\
S_{Y}:=- & \sum_{\underline{i}, \underline{j}} P_{X, Y}\left(x_{\underline{i}}, y_{\underline{j}}\right) \log P_{Y}\left(y_{\underline{j}}\right), \\
& \text { the total information content received by Bob ; }
\end{aligned}
$$




$$
S_{X}:=-\sum_{\underline{i}, \underline{j}} P_{X, Y}\left(x_{\underline{i}}, y_{\underline{j}}\right) \log P_{X}\left(x_{\underline{i}}\right),
$$

the total information content in Alice's message ;

$$
S_{X \mid Y}:=S(X \mid Y)=\sum_{\underline{j}} P_{Y}\left(y_{\underline{j}}\right) S_{X \mid Y=y_{j}},
$$

the conditional entropy .

Using such formulas, one prove that (this can be violated by quantum systems)

$S(X \mid Y)=S_{X Y}-S_{Y} \geq 0$

and the mutual information between $X$ and $Y$ (a measure of how much we learn about $X$ observing $Y$ )

$I(X ; Y):=S_{X}-S_{X Y}+S_{Y} \geq 0$.

Now, let us analyse another type of communications between Alice and Bob. We suppose that they are research scientists and know advanced differential geometry, classical mechanics, information theory, and theory of geometric flows. Alice sends to Bob not only simple messages consisting from letters and density probabilities but messages encoding that (in her world ) she study geometric flow evolution processes of a mechanical system of type $\widetilde{A}=\widetilde{A}[\widetilde{\mathbf{g}}(\tau)]$, or ${ }^{\prime} \widetilde{A}={ }^{\prime} \widetilde{A}\left[{ }^{\prime} \widetilde{\mathbf{g}}(\tau)\right]$, determined by flows of Hessian metrics. Bob will receive Alice's message (it may be a short letter) and knows that Alice plays a game with geometric flow modeling. We denote Bob's geometric evolution systems as $\widetilde{B}=\widetilde{B}\left[{ }_{1} \widetilde{\mathbf{g}}(\tau)\right]$, or ${ }^{~} \widetilde{B}={ }^{\prime} \widetilde{B}\left[{ }_{1}^{1} \widetilde{\mathbf{g}}(\tau)\right]$. In elaborating such GIF models, Alice and Bob could work or not with probability densities. In principle, the thermodynamic generating functions $\widetilde{\mathcal{Z}}[\widetilde{\mathbf{g}}(\tau)]$ and/or ' $\widetilde{\mathcal{Z}}\left[{ }^{\prime} \widetilde{\mathbf{g}}(\tau)\right]$ from $(35)$ can be considered as geometric flow analogs of probability densities but they may use directly the W-entropy $\widetilde{\mathcal{W}}(27)$, or ' $\widetilde{\mathcal{W}}(29)$, and, respectively, for ansambles of Hessian metrics, by $[\widetilde{\mathcal{E}}, \widetilde{\mathcal{S}}, \widetilde{\eta}]$ (36), or $\left[{ }^{\prime} \widetilde{\mathcal{E}}, ' \widetilde{\mathcal{S}},{ }^{\prime} \tilde{\eta}\right]$ (37). For simplicity, we analyze here how they may GIF-communicate using instead of messages with random letters certain geometric flow transfers of information encoding concepts of mechanical dual phase spacetimes for Lorentz cotangent bundles. In such a case, we have to use the geometric flow thermodynamic entropy ' $\widetilde{\mathcal{S}}\left[{ }^{\prime} \widetilde{\mathbf{g}}(\tau)\right]$ associated to W-entropy ' $\widetilde{\mathcal{W}}\left[{ }^{\prime} \mathbf{g}(\tau)\right]$ and formulas considered in Sect. 3.1.2. We shall use also geometric flow models on $T^{*} \mathbf{V} \otimes T^{*} \mathbf{V}$ with one cotangent bundle for Alice and another one for Bob. The local coordinates on such products of cotangent bundles are labeled ( $\left.u,{ }_{1}^{1} u\right)$ and the normalizing functions are of type ${ }_{A B}^{\prime} \widetilde{f}\left({ }^{\prime} u,{ }_{1} u\right)$. The canonical d-metric structure on such tensor products of phase spacetimes is of type

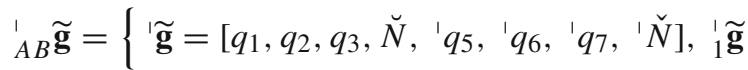

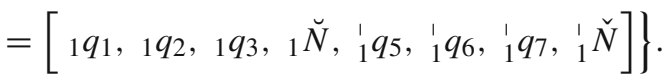

Respectively, we consider a canonical d-connection ${ }_{A B}^{\prime} \widetilde{\mathbf{D}}=$ ${ }^{\prime} \widetilde{\mathbf{D}}+{ }_{B}{ }_{B} \widetilde{\mathbf{D}}$ and respective scalar curvature ${ }_{s A B}^{1} \widetilde{R}={ }_{s}^{1} \widetilde{R}+{ }_{s 1}^{1} \widetilde{R}$. We work with ' $\widetilde{\mathcal{S}}[\widetilde{A}]$ and $' \widetilde{\mathcal{S}}[\widetilde{B}]$ defined by respective formulas for ${ }^{\prime} \widetilde{\mathbf{g}}(\tau)$ and ${ }_{1}^{1} \widetilde{\mathbf{g}}(\tau)$ as in (37). They are analogs of $S_{X}$ and $S_{Y}$ in above formulas. As an analog of $S_{X Y}$ for GIF, we consider the thermodynamic generating function (as a generalization of (35))

$$
\begin{aligned}
& { }_{A B} \widetilde{\mathcal{Z}}\left[{ }^{\prime} \widetilde{\mathbf{g}}(\tau),{ }_{1}^{\prime} \widetilde{\mathbf{g}}(\tau)\right]=\widetilde{\int}_{1} \int_{1} e^{-{ }_{A B} \tilde{f}} \\
& \times \sqrt{\left|{ }^{\prime} \widetilde{\mathbf{g}}_{\alpha \beta}\right|} \sqrt{{ }_{\mid}^{\prime}{ }_{1}^{\prime} \widetilde{\mathbf{g}}_{\alpha \beta} \mid} d^{8}{ }^{\prime} u d^{8}{ }_{1}^{\prime} u\left(-{ }_{A B} \tilde{f}+32\right),
\end{aligned}
$$$$
\text { for } T^{*} \mathbf{V} \otimes \mathbf{T}^{*} \mathbf{V} \text {, }
$$

and resulting entropy function

$$
\begin{aligned}
& { }_{A B}{ }_{B} \widetilde{\mathcal{S}}={ }^{\prime} \widetilde{\mathcal{S}}[\tilde{A}, \widetilde{B}]=-\tilde{\int}^{\prime} \tilde{\int}^{\tilde{\int}} e^{-{ }_{A B}{ }_{A B} \tilde{f}} \\
& \times \sqrt{\left|q_{1} q_{2} q_{3} \breve{N}{ }^{\prime} q_{5}{ }^{\prime} q_{6}{ }^{\prime} q_{7}{ }^{\prime} \check{N}\right|} \\
& \times \sqrt{\left|{ }_{1} q_{1} 1{ }_{1} q_{2}{ }_{1} q_{3}{ }_{1} \breve{N}_{1}^{1} q_{5}{ }_{1}^{1} q_{6}{ }_{1}^{1} q_{7}{ }_{1}^{1} \check{N}\right|} \delta^{8}{ }^{\prime} u d^{8}{ }_{1} u \\
& \times\left[\tau\left({ }_{s}^{1} \widetilde{R}+{ }_{s 1}^{1} \widetilde{R}+\left|{ }^{\prime} \widetilde{\mathbf{D}}_{A B}{ }_{A B} \tilde{f}+{ }_{1}^{1} \widetilde{\mathbf{D}}{ }_{A B} \tilde{f}\right|^{2}\right)+{ }_{A B} \tilde{f}-32\right] \text {. }
\end{aligned}
$$

Using such formulas, we claim that for GIFs the formulas for the conditional entropy (37) and mutual information (37) are respectively generalized

$$
\begin{aligned}
& \widetilde{\mathcal{S}}[\widetilde{A} \mid \widetilde{B}]:={ }_{A B}{ }_{A B} \widetilde{\mathcal{S}}-{ }^{\prime} \widetilde{\mathcal{S}}[\widetilde{B}] \geq 0 \text { and } \\
\prime & \widetilde{\mathcal{J}}[\widetilde{A} ; \widetilde{B}]:={ }^{\prime} \widetilde{\mathcal{S}}[\widetilde{A}]-{ }_{A B}{ }_{A B} \widetilde{\mathcal{S}}+{ }^{\prime} \widetilde{\mathcal{S}}[\widetilde{B}] \geq 0 .
\end{aligned}
$$

Similar claims can be formulated if we use the W-entropy ' $\widetilde{\mathcal{W}}(29)$ :

$$
\begin{aligned}
{ }^{\prime} \widetilde{\mathcal{W}}[\widetilde{A} \mid \widetilde{B}] & :={ }_{A B}{ }_{A B} \widetilde{\mathcal{W}}-{ }^{\prime} \widetilde{\mathcal{W}}[\widetilde{B}] \geq 0 \text { and }{ }^{\prime} \widetilde{\mathcal{J}}[\widetilde{A} ; \widetilde{B}] \\
& :={ }^{\prime} \mathcal{\mathcal { W }}[\widetilde{A}]-{ }_{A B}{ }_{A B} \widetilde{\mathcal{W}}+{ }^{\prime} \tilde{\mathcal{W}}[\widetilde{B}] \geq 0,
\end{aligned}
$$

with respective formulas computed for the W-entropy instead of the S-entropy in the standard probability theory. For relativistic information flows, such formulas can be applied without additional assumptions on formulating associated statistical thermodynamic models. ${ }^{10}$

\footnotetext{
10 Let us explain why we use the word "claim" for these formulas. In principle, the conditions of non-negativity of respective values can be violated if Alice sends to Bob GIFs as solutions, for instance, of generalized R. Hamilton geometric flow equations (45). For such variants, we use the claims (51) and (52) as criteria for selecting physically realistic and viable solutions for the information theory of geometric flow evolution of W. Hamilton mechanical systems. Nevertheless, working on cotangent Lorentz bundles, such claims can be transformed into theorems and proven if we consider a causal axiomatic approach Footnote 10 continued

to Finsler-Lagrange-Hamilton theories elaborated in [34,35]. Here we sketch the idea and key steps for proving such formulas. For physicists, such formulas seem to be natural ones; rigorous mathematical proofs require hundreds of pages and application of a corresponding interference of methods outlined in [1,13-15] together with [37,38,47-49] and, for nonholonomic configurations, in our works [19,23-29] . The
} 
Finally, we note that above formulas can be defined and proven respectively, and in similar forms, on $T \mathbf{V}, T \mathbf{V} \otimes \mathbf{T V}$, and other tensor products and lower dimension projections involving Lagrange generating functions. For instance,

$$
\begin{aligned}
\widetilde{\mathcal{S}}[\widetilde{A} \mid \widetilde{B}] & :={ }_{A B} \widetilde{\mathcal{S}}-\widetilde{\mathcal{S}}[\widetilde{B}] \geq 0 \text { and } \widetilde{\mathcal{J}}[\widetilde{A} ; \widetilde{B}] \\
& :=\widetilde{\mathcal{S}}[\widetilde{A}]-{ }_{A B} \widetilde{\mathcal{S}}+\widetilde{\mathcal{S}}[\widetilde{B}] \geq 0 ; \\
\widetilde{\mathcal{W}}[\widetilde{A} \mid \widetilde{B}] & :={ }_{A B} \widetilde{\mathcal{W}}-\widetilde{\mathcal{W}}[\widetilde{B}] \geq 0 \text { and } \widetilde{\mathcal{J}} \widetilde{\mathcal{W}}[\widetilde{A} ; \widetilde{B}] \\
& :=\widetilde{\mathcal{W}}[\widetilde{A}]-{ }_{A B} \widetilde{\mathcal{W}}+\widetilde{\mathcal{W}}[\widetilde{B}] \geq 0 .
\end{aligned}
$$

Such values can satisfy certain Legendre conditions and duality conditions to respective formulas (51) and (51) and Wanalogs. The models for cotangent bundles are important for elaborating quantum mechanical theories of GIFs with Hamilton generating functions. In their turn, the GIF models on tangent bundles are important for encoding quantum field theories formulated using the Lagrange formalism.

\subsubsection{Relative GIF entropy and monotonicity}

In the standard probability theory, the concept of relative entropy is introduced if (for a random variable $X$ ) there are considered two probability distributions $P_{X}$ and $Q_{X}$, where for $X=x_{i}$, labeled by $\underline{i}=\{1,2, \ldots s\}$, one obtains $p_{i}=$ $P_{X}\left(x_{\underline{i}}\right)$ and $q_{\underline{i}}=Q_{X}\left(x_{\underline{i}}\right)$, let say, for some long messages with $\underline{N}$ letters. The key issue is to decide which distribution describe a random process more realistically. The relative entropy per observation (or Kullback-Liebler divergence) is defined $S\left(P_{X} \| Q_{X}\right):=\sum_{i} p_{\underline{i}}\left(\log p_{\underline{i}}-\log q_{\underline{i}}\right) \geq 1$ under assumption that $N S\left(P_{X} \| Q_{X}\right) \gg 1$. This is an asymmetric value on $P_{X}$ and $Q_{X}$ and measure the difference between these two probability distributions when we consider that $P_{X}$ is a correct answer and $Q_{X}$ is an initial hypothesis.

Let us study a pair of random variables $X$ and $Y$ for which we consider two probability distributions. The fist one is a possible correlated joint distribution

$$
\begin{aligned}
P_{X, Y}\left(x_{\underline{i}}, y_{\underline{j}}\right) \text { and } P_{X}\left(x_{\underline{i}}\right):=\sum_{\underline{j}} P_{X, Y}\left(x_{\underline{i}}, y_{\underline{j}}\right), P_{Y}\left(y_{\underline{j}}\right) \\
:=\sum_{\underline{i}} P_{X, Y}\left(x_{\underline{i}}, y_{\underline{j}}\right) .
\end{aligned}
$$

W-entropy and respective thermodynamic values can be defined on a 3-d hypersurface as in (40), (41) and (42), and then extended for evolution on a time like curve to formulas (38) and (39). Then the formulas are dualized to momentum type local coordinates on some open regions on $T^{*} \mathbf{V} \otimes \mathbf{T}^{*} \mathbf{V}$. Such causal curves can be defined to cover a subspace on respective phase spacetimes, their tensor products, and projections on lower dimensions. Here we note that in any point of a causal curve in $T^{*} \mathbf{V}$ and related tensor products/ projection spaces and subspaces we can define entopies of type (48). This way, the geometric flow information values can be completed with certain random variables. Alice's letters to Bob will encode not only GIFs but also random bit information processes. We can associate entropies of type ' $\widetilde{\mathcal{W}}$ and/or ' $\widetilde{\mathcal{S}}$ to probabilistic entropies.
A second probability distribution $Q_{X, Y}\left(x_{i}, y_{j}\right)=P_{X}\left(x_{i}\right)$ $P_{Y}\left(y_{j}\right)$ can be defined to ignore correlations between $X$ and $Y$. In a general context, $Q_{X, Y}\left(x_{i}, y_{j}\right)$ can be with correlations when $Q_{X}\left(x_{\underline{i}}\right):=\sum_{j} Q_{X, Y}\left(x_{\underline{i}}, y_{\underline{j}}\right)$. For more general constructions, we can introduce three random variables $X, Y, Z$ described by a joint probability distribution and related values:

$$
\begin{aligned}
P_{X, Y, Z}\left(x_{\underline{i}}, y_{\underline{j}}, z_{\underline{k}}\right) \text { and } P_{X}\left(x_{\underline{i}}\right):=\sum_{\underline{j}, \underline{k}} P_{X, Y, Z}\left(x_{\underline{i}}, y_{\underline{j}}, z_{\underline{k}}\right), \\
P_{Y, Z}\left(y_{\underline{j}}, z_{\underline{k}}\right):=\sum_{\underline{i}} P_{X, Y, Z}\left(x_{\underline{i}}, y_{\underline{j}}, z_{\underline{k}}\right) .
\end{aligned}
$$

If we forget the correlations between $X$ and $Y Z$, we define $Q_{X, Y, Z}\left(x_{\underline{i}}, y_{j}, z_{\underline{k}}\right):=P_{X}\left(x_{\underline{i}}\right) P_{Y, Z}\left(y_{j}, z_{\underline{k}}\right)$. Other type values can be defined if we observe the subsystem $X Y$, when

$$
\begin{aligned}
P_{X, Y}\left(x_{\underline{i}}, y_{\underline{j}}\right) & :=\sum_{\underline{k}} P_{X, Y, Z}\left(x_{\underline{i}}, y_{\underline{j}}, z_{\underline{k}}\right), Q_{X, Y}\left(x_{\underline{i}}, y_{\underline{j}}\right) \\
& :=\sum_{\underline{k}} Q_{X, Y, Z}\left(x_{\underline{i}}, y_{\underline{j}}, z_{\underline{k}}\right)=P_{X}\left(x_{\underline{i}}\right) P_{Y}\left(y_{\underline{j}}\right) .
\end{aligned}
$$

Now, we can calculate the relative entropy $S$ and mutual information $I$ between two distributions

$$
\begin{aligned}
S\left(P_{X} \| Q_{X}\right):= & \sum_{i, j} P_{X, Y}\left(x_{\underline{i}}, y_{\underline{j}}\right) \\
& \times\left[\log P_{X, Y}\left(x_{\underline{i}}, y_{\underline{j}}\right)-\log \left(P_{X}\left(x_{\underline{\underline{x}}}\right) P_{Y}\left(y_{\underline{j}}\right)\right)\right] \\
= & S_{X}-S_{X Y}+S_{Y}=I(X ; Y) ; \\
S\left(P_{X, Y} \| Q_{X, Y}\right):= & S_{X}-S_{X Y}+S_{Y}=I(X ; Y) ; \\
S\left(P_{X, Y, Z} \| Q_{X, Y, Z}\right):= & S_{X Y}-S_{X Y Z}-S_{Y Z}=I(X ; Y Z) .
\end{aligned}
$$

In result, one proves by explicit calculations such properties

$I(X ; Y):=S_{X}+S_{Y}-S_{X Y} \geq 0$, subadditivity of entropy ;

$$
S\left(P_{X, Y} \| Q_{X, Y}\right) \geq S\left(P_{X} \| Q_{X}\right), S\left(P_{X, Y, Z} \| Q_{X, Y, Z}\right)
$$

$\geq S\left(P_{X, Y} \| Q_{X, Y}\right)$, monotonicity of relative entropy.

There is also the condition of strong subadditivity

$$
\begin{aligned}
& S_{X}-S_{X Y Z}-S_{Y Z} \geq S_{X}-S_{X Y}+S_{Y}, \\
& \quad \text { or } S_{X Y}+S_{Y Z} \geq S_{Y}+S_{X Y Z},
\end{aligned}
$$

which is equivalent for the condition of monotonity of mutual information $I(X ; Y Z) \geq I(X ; Y)$.

Above formulas for $S$ and $I$ can be generalized for respective relative entropy and mutual information of geometric flows of mechanical systems (for simplicity, we consider formulas generated by certain relativistic Hamilton generating functions $H(x, p))$. For such evolution systems, there are considered ${ }_{A}{ }_{A} \widetilde{\mathcal{Z}}:=\mid \widetilde{\mathcal{Z}}\left[{ }^{\prime \mathbf{g}}(\tau)\right]$ and ${ }_{B}{ }_{B} \widetilde{\mathcal{Z}}:={ }_{1}^{1} \widetilde{\mathcal{Z}}\left[{ }_{1}{ }_{1} \widetilde{\mathbf{g}}(\tau)\right]$, see (35), as analogs of $p_{i}=P_{X}\left(x_{i}\right)$ and $q_{i}=Q_{X}\left(x_{i}\right)$, see also formulas in the previous section. In general, there are considered three evolution flow canonical mechanical systems $\widetilde{A}, \widetilde{B}, \widetilde{C}$. In result, we claim (and can prove following 
the method sketched in footnote 10) by explicit integral $\mathrm{N}$ adapted calculations on $T^{*} \mathbf{V} \otimes \mathbf{T}^{*} \mathbf{V} \otimes \mathbf{T}^{*} \mathbf{V}$ such properties

$$
\text { ' } \widetilde{\mathcal{J}}[\widetilde{A} ; \widetilde{B}]:=' \widetilde{\mathcal{S}}[\widetilde{A}]-{ }_{A B}{ }_{A B} \widetilde{\mathcal{S}}+{ }^{\prime} \widetilde{\mathcal{S}}[\widetilde{B}] \geq 0,
$$

subadditivity of entropy;

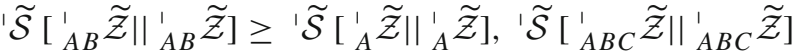

$$
\begin{aligned}
& \geq ' \widetilde{\mathcal{S}}\left[{ }_{A B}^{1} \tilde{\mathcal{Z}} \|_{A B}{ }_{A B} \widetilde{\mathcal{Z}}\right] \text {, } \\
& \text { monotonicity of relative entropy . }
\end{aligned}
$$

The conditions of strong subadditivity for GIF entropies are claimed

$$
\begin{aligned}
& { }_{A} \widetilde{\mathcal{S}}-{ }_{A B C} \widetilde{\mathcal{S}}-{ }_{B C}{ }_{B C} \widetilde{\mathcal{S}} \geq{ }_{A}{ }_{A} \widetilde{\mathcal{S}}-{ }_{A B}{ }_{A B} \widetilde{\mathcal{S}}+{ }_{B}{ }_{B} \widetilde{\mathcal{S}}, \text { or }{ }_{A B}{ }_{A B} \widetilde{\mathcal{S}} \\
& +{ }_{B C} \widetilde{\mathcal{S}} \geq{ }_{B}{ }_{B} \widetilde{\mathcal{S}}+{ }_{A B C}{ }_{A B} \widetilde{\mathcal{S}} .
\end{aligned}
$$

In equivalent form, these formulas can be written as the condition of monotonicity of GIFs mutual information,

$$
\text { ' } \widetilde{\mathcal{J}}[\widetilde{A} ; \widetilde{B} \widetilde{C}] \geq ' \widetilde{\mathcal{J}}[\widetilde{A} ; \widetilde{B}] .
$$

Above formulas involve, for instance, the thermodynamic generating function (as a generalization of (35))

$$
\begin{aligned}
& { }_{A B C}{ } \widetilde{\mathcal{Z}}\left[{ }^{\prime} \widetilde{\mathbf{g}}(\tau),{ }_{1}^{\prime} \widetilde{\mathbf{g}}(\tau),{ }_{2}^{1} \widetilde{\mathbf{g}}(\tau)\right] \\
& =\tilde{\int}_{1} \tilde{1} \tilde{\int} \begin{array}{l}
1 \\
2
\end{array} e^{-{ }_{A B C} \tilde{f}} \\
& \times \sqrt{\left|\widetilde{\mathbf{g}}_{\alpha \beta}\right|} \sqrt{\left|{ }_{1}^{1} \widetilde{\mathbf{g}}_{\alpha \beta}\right|} \sqrt{\left|{ }_{2}^{1} \widetilde{\mathbf{g}}_{\alpha \beta}\right|} d^{8}{ }^{\prime} u d^{8}{ }_{1} u d^{8}{ }_{2} u \\
& \times\left(-{ }_{A B C}{ }_{A B} \tilde{f} 48\right) \text {, for } T^{*} \mathbf{V} \otimes \mathbf{T}^{*} \mathbf{V} \otimes \mathbf{T}^{*} \mathbf{V} \text {, }
\end{aligned}
$$

with a normalizing function ${ }_{A B C} \tilde{f}\left({ }^{\prime} u,{ }_{1}^{1} u,{ }_{2}^{1} u\right)$, when the local coordinates on such such products of cotangent bundles

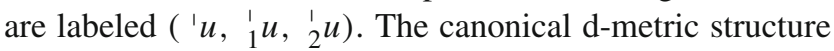
on such tensor products of phase spacetimes is of type

$$
\begin{aligned}
& { }_{A B C}^{\prime} \widetilde{\mathbf{g}}=\left\{\text { ' } \widetilde{\mathbf{g}}=\left[q_{1}, q_{2}, q_{3}, \breve{N},{ }^{\prime} q_{5},{ }^{\prime} q_{6},{ }^{\prime} q_{7},{ }^{\prime} \check{N}\right],{ }_{1}\right.
\end{aligned}
$$

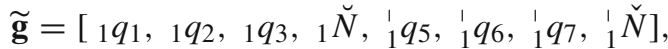

$$
\begin{aligned}
& \left.{ }_{2}^{1} \widetilde{\mathbf{g}}=\left[{ }_{2} q_{1},{ }_{2} q_{2},{ }_{2} q_{3},{ }_{2} \breve{N},{ }_{2}^{1} q_{5},{ }_{2}^{1} q_{6},{ }_{2}^{1} q_{7},{ }_{2} \tilde{N}\right]\right\} .
\end{aligned}
$$

We can consider a canonical d-connection ${ }_{A B C}{ }_{B C} \widetilde{\mathbf{D}}={ }^{\prime} \widetilde{\mathbf{D}}+{ }_{B}{ }_{B} \widetilde{\mathbf{D}}$ $+{ }_{C}^{\prime} \widetilde{\mathbf{D}}$ and respective scalar curvature ${ }_{s A B C}^{{ }_{1}} \widetilde{R}={ }_{s}^{1} \widetilde{R}+{ }_{s 1}^{1} \widetilde{R}+$ ${ }_{s 2}{ }_{2} \widetilde{R}$. The resulting entropy function

$$
\begin{aligned}
& { }_{A B C} \widetilde{\mathcal{S}}=' \widetilde{\mathcal{S}}[\widetilde{A}, \widetilde{B}, \widetilde{C}]=-\tilde{\int}_{1} \tilde{\int}_{1} \tilde{\int}_{2} e^{-{ }_{A B C} \widetilde{f}} \\
& \times \sqrt{\left|q_{1} q_{2} q_{3} \breve{N}^{\prime} q_{5}{ }^{\prime} q_{6}{ }^{\prime} q_{7}{ }^{\prime} \check{N}\right|} \\
& \times \sqrt{\left|{ }_{1} q_{11}{ }_{1} q_{2}{ }_{1} q_{3}{ }_{1} \breve{N}_{1}^{\prime} q_{5}{ }_{1}^{1} q_{6}{ }_{1}^{\prime} q_{7}{ }_{1}{ }_{1} \check{N}\right|} \\
& \times \sqrt{\left|{ }_{2} q_{1}{ }_{2} q_{2} 2 q_{3}{ }_{2} \breve{N}_{2}^{1} q_{5}{ }_{2}^{1} q_{6}{ }_{2}^{1} q_{7}{ }_{2}{ }_{2} \tilde{N}\right|} \delta^{8} u d^{8}{ }_{1} u d^{8}{ }_{2} u \\
& \times\left[\tau \left({ }_{s}^{1} \widetilde{R}+{ }_{s 1}^{1} \widetilde{R}++{ }_{s 2}^{1} \widetilde{R}+\mid{ }^{\prime} \widetilde{\mathbf{D}}_{A B C}^{\prime} \widetilde{f}+{ }_{1} \widetilde{\mathbf{D}}_{A B C}^{\prime} \tilde{f}\right.\right. \\
& \left.\left.+\left.{ }_{2}^{1} \widetilde{\mathbf{D}}_{A B C}{ }_{A B} \tilde{f}\right|^{2}\right)+{ }_{A B C}{ }_{A B} \tilde{f}-48\right] \text {. }
\end{aligned}
$$

Similar formulas can be derived for W-entropies and for Lagrange GIFs on $T \mathbf{V} \otimes \mathbf{T V} \otimes \mathbf{T V}$.
We conclude this introduction to the GIF theory of canonical classical mechanical systems with two remarks: First, such constructions can be generalized for stochastic maps and nonholonomic flow evolution and kinetic processes of Lagrange-Hamilton systems as we studied in [53-55]. Here, we shall analyse a QGIF analog when the quantum relative entropy is monotonic in any quantum channel, including those associated to evolution of Hamiltonian quantum mechanical systems.

Second, we shown that we are able both in the probability theory and for geometric flow models to define conditional on some observation entropies. There is not a good analog of the probability conditional distribution in the quantum mechanical case. Nevertheless, there is a miracle that many conclusions have quantum analogs [38]. For GIFs of mechanical Hamilton systems with a $H(\tau, x, p)$, this is not a miracle because the flow evolution of Hessian Hamilton metrics ' $\widetilde{g}^{a b}(\tau, x, p):=\frac{1}{2} \partial^{2} H / \partial p_{a} \partial p_{b}$ (4) and respective canonical d-metrics ' $\widetilde{\mathbf{g}}(\tau)$ (14) are characterized by welldefined concepts of W-entropy ' $\widetilde{\mathcal{W}}(27)$ and respective thermodynamical variables $\left[{ }^{\prime} \mathcal{\mathcal { E }}, ' \widetilde{\mathcal{S}}, ' \widetilde{\eta}\right]$ (37). In result, we can introduce GIF formulas for conditional entropy and mutual entropy and their $\mathrm{W}$-analogs. For quantum developments in next section, we shall speculate on strong subadditivity of quantum entropy which holds also for quantum analogs of mechanical Hamilton systems.

\subsection{Basic ingredients of the quantum geometric} information flow theory

The goal of this section is to analyze how the main concepts and formulas for GIFs of mechanical systems can be extended to quantum theory and formulate an approach to the theory of QGIFs. We note that a noncommutative version of geometric flow theory was elaborated in [23]. Those results can be extended for elaborating noncommutative models of quantum information theory. In a more simplified approach, we can consider quantum mechanical models, and respective quantum geometric flows, by quantizing certain relativistic mechanical Hamiltonians $H(\tau, x, p)$, when in the quasiclassical limits the geometric mechanics theory with Hessian metrics ${ }^{\prime} \widetilde{g}^{a b}(\tau, x, p)$ emerges. In this work, the main goal is to elaborate on quantum information theory for geometric flows of mechanical systems characterized by geometric thermodynamical data $[' \widetilde{\mathcal{W}}$; ' $\widetilde{\mathcal{E}}, ' \widetilde{\mathcal{S}}, ' \widetilde{\eta}]$, see (27) and (37).

\subsubsection{Density matrices and properties of quantum entropies for GIFs}

\section{Statistical density matrix for relativistic mechanical Hamilton flows}

The thermodynamic generating function ' $\widetilde{\mathcal{Z}}\left[{ }^{\prime} \widetilde{\mathbf{g}}(\tau)\right]$ (35) with canonical geometric objects determined by a Hamilton 
function $\widetilde{H}$, see also Sect. 3.1.2, can be used for defining the state density

$$
{ }^{\prime} \widetilde{\sigma}(\beta, \widetilde{H}, ' \widetilde{\mathbf{g}})={ }^{\prime} \widetilde{\mathcal{Z}}^{-1} e^{-\beta \widetilde{H}},
$$

with $\beta=1 / T, \tau=T$, as a classical analog of the density matrix in quantum mechanics. The relative entropy between any state density ' $\widetilde{\rho}\left(\beta, \widetilde{H},{ }_{1}^{\prime} \widetilde{\mathbf{g}}\right)$ and ${ }^{\prime} \widetilde{\sigma}(\beta, \widetilde{H}, \quad \widetilde{\mathbf{g}})$ is computed for a prescribed measure $\omega(\tilde{H})$, for instance, on a cotangent Lorentz bundle with $E$ considered as a thermodynamical energy parameter.

Using formulas (33) and (32), we define for the conditional entropy for geometric flows of Hamilton mechanical systems

$$
{ }^{\prime} \widetilde{\mathcal{S}}\left({ }^{\prime} \tilde{\rho} \| ' \widetilde{\sigma}\right)=\beta\left[{ }^{\prime} \widetilde{\mathcal{F}}\left({ }^{\prime} \tilde{\rho}\right)-{ }^{\prime} \tilde{\mathcal{F}}(' \widetilde{\sigma})\right],
$$

where the free energy corresponding to ' $\widetilde{\rho}$ is $\mid \widetilde{\mathcal{F}}\left({ }^{\prime} \tilde{\rho}\right):=$ $' \widetilde{\mathcal{E}}\left({ }^{\prime} \widetilde{\rho}\right)-T^{\prime} \widetilde{\mathcal{S}}\left({ }^{\prime} \tilde{\rho}\right)$. In these formulas, the average energy is computed $' \widetilde{\mathcal{E}}(' \widetilde{\rho})=\int{ }^{\prime} \widetilde{\rho} \widetilde{H} d \omega(\widetilde{H})$ (i.e. using the density matrix ' $\widetilde{\rho})$ and the thermodynamic entropy is $' \widetilde{\mathcal{S}}\left({ }^{\prime} \widetilde{\rho}\right):=$ $\beta^{\prime} \mathcal{\mathcal { E }}\left({ }^{\prime} \widetilde{\rho}\right)+\log '^{\prime} \widetilde{\mathcal{Z}}(' \widetilde{\rho})$. Both values ' $\widetilde{\mathcal{E}}\left({ }^{\prime} \widetilde{\rho}\right)$ and $' \widetilde{\mathcal{S}}(' \widetilde{\rho})$ can be written equivalently to (37). We note that if $\log ' \tilde{\mathcal{Z}}$ is independent on ' $\widetilde{\rho}$ (as we consider in above formulas) we have ' $\widetilde{\mathcal{S}}\left({ }^{\prime} \tilde{\sigma} \| ' \widetilde{\sigma}\right)=0$.

In this section, we elaborate on how GIFs of classical mechanical systems can be generalized to QGIFs using basic concepts of quantum mechanics, QM, and information theory. QM involves probabilities not as classical probability distributions for a quantum state but, in general, as densities matrices. Certain special QM systems can be described by pure states. Nevertheless, to study quantum models of GIFs systems is necessary to consider density matrices as quantum analogs of state densities of type ' $\widetilde{\sigma}(54)$.

\section{Density matrix for quantum information theory and asso- ciated Hamilton mechanical systems}

In an idealized case, a Hamiltonian GIF system $\widetilde{A}=$ $[' \widetilde{\mathcal{E}}, ' \widetilde{\mathcal{S}}, ' \widetilde{\eta}]$ (37) can be described by a Hilbert space $\widetilde{\mathcal{H}}_{A}$. A state vector $\widetilde{\psi}_{A} \in \widetilde{\mathcal{H}}_{A}$ can be defined as infinite dimensional complex vector solving the Schrödinger equation with a Hamiltonian $\widehat{H}$ taken as a well-defined quantum version of a canonical Hamiltonian $\widetilde{H}$. In the quasi-classical limit, from a quantum mechanical model with $\widehat{H}$, we obtain a relativistic $\widetilde{H}$ and respective Hessian ' $\widetilde{g}^{a b}(x, p)$ (4) and canonical d-metric ' $\widetilde{\mathbf{g}}$ (15) (from which "non-tilde" d-metrics

'g (10) emerge for general frame and coordinate transforms on a $T \mathbf{V}$ ). We can consider unitary transforms of type $\widetilde{\psi}_{A} \rightarrow U \psi_{A}$ and describe the system $\widetilde{A}$ in an abstract Hilbert space $\mathcal{H}_{\widetilde{A}}$ (we put tilde on certain symbols if it is necessary to emphasize that the constructions are related to quantization of a canonical mechanical Hamiltonian system). For applications in the information theory, a Hilbert space is approximated to a complex vector space of dimension $N$ with Hermitian product, see details in $[37,38]$.
We can consider a complementary system $B$ (we write $\widetilde{B}$ if it is a quantum mechanical analog of a classical Hamilton mechanics) with an associate Hilbert space $\mathcal{H}_{B}$, or $\mathcal{H}_{\widetilde{B}}$, with state vectors of type $\psi_{B} \in \mathcal{H}_{B}$ and/or unitary transforms of type $\widetilde{\psi}_{B} \rightarrow \psi_{B} V \in \mathcal{H}_{\widetilde{B}}$. The combined Hilbert space is defined as a tensor product, $\mathcal{H}_{A} \otimes \mathcal{H}_{B}$ and/or $\mathcal{H}_{\widetilde{A}} \otimes \mathcal{H}_{\widetilde{B}}$. The state vectors for the combined system are of type

$\psi_{A B}=\psi_{A} \otimes \psi_{B} \in \mathcal{H}_{A B}=\mathcal{H}_{A} \otimes \mathcal{H}_{B}$,

where, for instance, $\psi_{B}=1_{B}$ is considered as the unity state vector. For such products, the predictions about a system $\widetilde{A}$ can be made using the state vector $\widetilde{\psi}_{A}$ and forgetting about the system $B$. In general, a generic pure state $\psi_{A B} \in \mathcal{H}_{A B}$ is not a tensor product vector but is "entangled". This means that if the respective dimension $\operatorname{dim} \mathcal{H}_{A}=\underline{N}$ and $\operatorname{dim} \mathcal{H}_{B}=\underline{M}$ then a generic state $\psi_{A B}$ is described by an $\underline{N} \times M$ matrix. In quantum information theory, it is considered that any pure state can be written as a Schmidt decomposition

$\psi_{A B}=\sum_{\underline{i}} \sqrt{p_{\underline{i}}} \psi_{\bar{A}}^{\underline{i}} \otimes \psi_{B}^{\underline{i}}$ or $\widetilde{\psi}_{A B}=\sum_{\underline{i}} \sqrt{p_{\underline{i}}} \widetilde{\psi}_{\bar{A}}^{\underline{i}} \otimes \widetilde{\psi}_{B}^{\underline{i}}$.

In such formulas, the state vectors are orthonormal: for instance, $<\psi_{A}^{i}, \psi_{A}^{\frac{j}{j}}>=<\psi_{B}^{i}, \psi_{B}^{j}>=\delta^{-} \underline{i}$, where $\delta^{\underline{i}} \underline{\underline{j}}$ is the Kronecker symbol. If $p_{i}>0$ and $\sum_{i} p_{i}=1$ (this is equivalent to the condition that, for instance, $\psi_{A B}$ is a unit vector), we can treat $p_{i}$ as probabilities. Here we note that $\psi_{A}^{i}$, or $\psi_{B}^{i}$, may not be bases of $\mathcal{H}_{A}$, or $\mathcal{H}_{B}$ (in principle, they may be not enough for such bases).

The quantum density matrix for a system $A$, or $\widetilde{A}$, is defined

$\rho_{A}:=\sum_{\underline{i}} p_{\underline{i}}\left|\psi_{\bar{A}}^{i}><\otimes \psi_{\bar{A}}^{i}\right|$ or $\rho_{\widetilde{A}}:=\sum_{\underline{i}} p_{\underline{i}}\left|\psi_{\widetilde{\widetilde{A}}}^{i}><\otimes \psi_{\widetilde{\widetilde{A}}}^{i}\right|$.

This operator is Hermitian and positive semi-definite, with trace $\operatorname{Tr}_{\mathcal{H}_{A}} \rho_{A}=\operatorname{Tr}_{\mathcal{H}_{\tilde{A}}} \rho_{\widetilde{A}}=1$. Using $\rho_{A}$, or $\rho_{\widetilde{A}}$, we can compute the expectation value of any operator $\mathcal{O}_{A}$, or $\mathcal{O}_{\widetilde{A}}$, following, for instance, the rules

$$
\begin{aligned}
<\mathcal{O}>_{A B} & =\left\langle\psi_{A B}\left|\mathcal{O}_{A} \otimes 1_{B}\right| \psi_{A B}\right\rangle \\
& =\sum_{\underline{i}} p_{\underline{i}}\left\langle\psi_{A}^{\frac{i}{i}}\left|\mathcal{O}_{A}\right| \psi_{A}^{i}\right\rangle\left\langle\psi_{B}^{i}\left|1_{B}\right| \psi_{B}^{i}\right\rangle= \\
\langle\mathcal{O}\rangle_{A} & =\sum_{\underline{i}} p_{\underline{i}}\left\langle\psi_{A}^{\frac{i}{A}}\left|\mathcal{O}_{A}\right| \psi_{A}^{\frac{i}{A}}\right\rangle=\operatorname{Tr}_{\mathcal{H}_{A}} \rho_{A} \mathcal{O}_{A} .
\end{aligned}
$$

In above formulas, we considered a bipartite system $A B$, or $\widetilde{A} \widetilde{B}$. Such systems are described in general form by quantum denstity matrices of type $\rho_{A B}$, or $\rho_{\widetilde{A} \widetilde{B}}$. Here we note that in the classical probability theory a bipartite system $X Y$ is described by a joint probability distribution $P_{X, Y}\left(x_{\underline{i}}, y_{j}\right)$, where $P_{X}\left(x_{\underline{i}}\right):=\sum_{j} P_{X, Y}\left(x_{\underline{i}}, y_{j}\right)$, see (53). For $A \bar{B}$ as a bipartite quantum system with Hilbert space $\mathcal{H}_{A} \otimes \mathcal{H}_{B}$, the 
density matrix $\rho_{A B}$ is defined in standard quantum mechanical form: Let us consider $\mid \underline{i}>_{A}, \underline{i}=1,2, \ldots, \underline{n}$ as an orthonormal basis of $\mathcal{H}_{A}$ and $\underline{b}>_{B}, \underline{b}=1,2, \ldots, \underline{m}$ as an orthonormal basis of $\mathcal{H}_{B}$. We write

$\rho_{A B}=\sum_{\underline{i}, \underline{i^{\prime}}, \underline{b}, \underline{b^{\prime}}} \rho_{i \underline{i}^{\prime} \underline{b} \underline{b}^{\prime}}\left|\underline{i}>_{A} \otimes\right| \underline{b}>_{B} \quad A<\underline{i}^{\prime}\left|\otimes{ }_{B}<\underline{b}^{\prime}\right|$.

For measurements of the system $A$, it is considered the reduced density matrix obtained by respective contracting of indices,

$$
\begin{aligned}
& \rho_{A}=\operatorname{Tr}_{\mathcal{H}_{B}} \rho_{A B}=\sum_{\underline{i}, \underline{i^{\prime}}, \underline{b}, \underline{\underline{b}}} \rho_{\underline{\underline{i}} \underline{i^{\prime}} \underline{\underline{b}} \underline{b} \underline{i}>_{A} \quad A<\underline{i}^{\prime} \mid,} \\
& \quad \text { for } \mid \underline{b}>_{B} \quad B \quad<\underline{b}=1 .
\end{aligned}
$$

In a similar form, it is defined $\rho_{B}=\operatorname{Tr}_{\mathcal{H}_{A}} \rho_{A B}$. Using such formulas, we can elaborate on quantum information theory (see reviews [37,38]) and develop the approach for QGIFs.

\section{Quantum density matrix for GIFs of mechanical Hamil- ton systems}

Using formulas (57), we can compute expectation values of a state density ' $\widetilde{\sigma}(54)$ and define a respective quantum density

$$
\begin{aligned}
& { }^{\prime} \widetilde{\sigma}_{A B}=\left\langle{ }^{\prime} \widetilde{\sigma}\right\rangle_{A B}=\left\langle\psi_{A B}\left|{ }^{\prime} \tilde{\sigma} \otimes 1_{B}\right| \psi_{A B}\right\rangle \\
& =\sum_{\underline{i}} p_{\underline{i}}\left\langle\psi_{A}^{i}\left|{ }^{i} \tilde{\sigma}\right| \psi_{A}^{i}\right\rangle\left\langle\psi_{B}^{i}\left|1_{B}\right| \psi_{B}^{i}\right\rangle \\
& { }^{\prime} \tilde{\sigma}_{A}=\left\langle{ }^{\prime} \tilde{\sigma}\right\rangle_{A}=\sum_{\underline{i}} p_{\underline{i}}\left\langle\psi_{A}^{i}\left|{ }^{\prime} \tilde{\sigma}\right| \psi_{A}^{i}\right\rangle=T r_{\mathcal{H}_{A}} \rho_{A}{ }^{\prime} \tilde{\sigma} .
\end{aligned}
$$

Here the density matrix $\rho_{A}$ is taken for computing the QGIF density matrix ' $\tilde{\sigma}_{A}$ determined by a state density of the thermodynamical model for GIFs of a classical mechanical Hamiltonian system ' $\widetilde{\sigma}$. For such systems, we can work directly with quantum density matrices ' $\widetilde{\sigma}_{A B}$ and ' $\widetilde{\sigma}_{A}$ and respective partial traces

$$
{ }^{\prime} \tilde{\sigma}_{A}=\operatorname{Tr}_{\mathcal{H}_{B}}{ }^{\prime} \tilde{\sigma}_{A B} \text { and }{ }^{\prime} \widetilde{\sigma}_{B}=\operatorname{Tr}_{\mathcal{H}_{A}}{ }^{\prime} \widetilde{\sigma}_{A B} \text {. }
$$

In coefficient form, we obtain such formulas

$$
\begin{aligned}
& { }^{\prime} \widetilde{\sigma}_{A B}=\sum_{\underline{i}, \underline{i^{\prime}}, \underline{b}, \underline{b^{\prime}}}\left|\tilde{\sigma}_{i \underline{i}^{\prime}} \underline{\underline{b} \underline{b}^{\prime}}\right| \underline{i}>_{A} \otimes\left|\underline{b}>_{B} \quad A<\underline{i}^{\prime}\right| \otimes{ }_{B}<\underline{b^{\prime}} \mid \\
& \text { and }{ }^{\prime} \tilde{\sigma}_{A}=\sum_{\underline{i}, \underline{\prime}, \underline{b}, \underline{b}, \underline{b}}\left|\tilde{\sigma}_{i i^{\prime}} \underline{b b} \underline{\underline{b}}\right| \underline{i}>_{A} \quad A<\underline{i}^{\prime} \mid .
\end{aligned}
$$

Let us discuss a concrete example with density matrices. Consider an isolated classical mechanical Hamitonian systems for which a QM model can be constructed. To describe thermodynamically the geometric flow evolution of both classical and quantum models we need respective state density and quantum density matrix. In a pure state formalism, the mathematical machinery gets bigger and bigger involving differential geometric concepts, quantum mechanics and probability theories. This can be organized as quantum information flow evolution model. Using a density matrix encoding the data for Hamilton mechanical system, we can compute respective thermodynamical values.

\subsubsection{Properties of entropies for QGIFs}

\section{The von Neumann entropy of density matrix for QGIFs of mechanical systems}

Using ' $\widetilde{\sigma}_{A}$, we can describe QGIF in a standard QM form when the respective von Neumann entropy is used instead of the Shannon entropy for a probability distribution,

$$
{ }_{q}^{\prime} \widetilde{\mathcal{S}}\left({ }^{\prime} \widetilde{\sigma}_{A}\right):=\operatorname{Tr}{ }^{\prime} \widetilde{\sigma}_{A} \log { }^{\prime} \widetilde{\sigma}_{A},
$$

where the trace is written in a simplified form without a label for the corresponding Hilbert space. We use a left label $q$ as "quantum" and emphasize that such an entropy is a quantum analog of ' $\widetilde{\mathcal{S}}$ used in the thermodynamic model for geometric flow evolution of Hamilton mechanical systems. The QGIF entropy ${ }_{q}^{\prime} \widetilde{\mathcal{S}}\left({ }^{\prime} \widetilde{\sigma}_{A}\right) \geq 0$ and is manifestly invariant under a unitary transformation ${ }^{\prime} \widetilde{\sigma}_{A} \rightarrow U$ ' $\widetilde{\sigma}_{A} U^{-1}$.

The quantum value ${ }_{q} \widetilde{\mathcal{S}}\left({ }^{\prime} \widetilde{\sigma}_{A}\right)$ has a purifying property which is typical for quantum information theory and does not have a classical analog. For a bipartite system $\widetilde{\psi}_{A B}=$ $\sum_{\underline{i}} \sqrt{p_{\underline{i}}} \widetilde{\psi}_{A}^{i} \otimes \widetilde{\psi}_{B}^{i}(56)$ and $\rho_{A}:=\sum_{\underline{i}} p_{\underline{i}}\left|\psi_{A}^{i}>\otimes<\psi_{A}^{i}\right|$, we write

$$
\begin{aligned}
& { }^{\prime} \widetilde{\sigma}_{A}:=\sum_{\underline{i}, \underline{i^{\prime}}, \underline{b}, \underline{b} \underline{\underline{b}}} \sum_{\underline{k}}^{1} \tilde{\sigma}_{\underline{i i^{\prime}} \underline{b} \underline{b}} p_{\underline{k}} A\left\langle\underline{i^{\prime}} \| \psi \frac{k}{A}\right\rangle\left\langle\otimes \psi \frac{k}{A} \| \underline{i}\right\rangle_{A}, \\
& { }^{\prime} \widetilde{\sigma}_{B}:=\sum_{\underline{j}, \underline{j^{\prime}}, \underline{b}, \underline{b}} \sum_{\underline{k}}^{\prime} \tilde{\sigma}_{j \underline{j} j^{\prime} \underline{b} \underline{b}} p_{\underline{k}} B\left\langle\underline{j^{\prime}} \| \psi \frac{k}{B}\right\rangle\left\langle\otimes \psi \frac{k}{B} \| \underline{j}\right\rangle_{B} .
\end{aligned}
$$

In both these formulas, we have the sam probabilities $p_{\underline{k}}$ even the matrices and bases are different. So, it is clear that ${ }_{q}^{1} \widetilde{\mathcal{S}}\left({ }^{\prime} \widetilde{\sigma}_{A}\right)={ }_{q}^{1} \widetilde{\mathcal{S}}\left({ }^{\prime} \widetilde{\sigma}_{B}\right)$, which proves that a system $A$ and a purifying system $B$ always have the same QGIF von Neumann entropy. This holds true if $\widetilde{A}$ is taken for GIFs of a mechanical Hamilton system.

Because ${ }_{q} \widetilde{\mathcal{S}}(' \widetilde{\sigma})$ is a typical von Neumann entropy, it has another very important concavity property. Let explain this for QGIFs because there are involved certain important features induced by geometric flow evolution. This mean that for any two density mechanical matrices ' $\widetilde{\sigma}_{1}$ and ' $\widetilde{\sigma}_{2}$ we can introduce ${ }^{\prime} \widetilde{\sigma}(\lambda)=\lambda{ }^{\prime} \widetilde{\sigma}_{1}+(1-\lambda)^{\prime} \widetilde{\sigma}_{2}$, for $0 \leq \lambda \leq 1$, and prove that $d^{2}{ }_{q}^{\prime} \widetilde{\mathcal{S}}(' \widetilde{\sigma}) / d \lambda^{2} \leq 0$. In result, one obtains ${ }_{q}^{\prime} \widetilde{\mathcal{S}}\left({ }^{\prime} \widetilde{\sigma}_{D}\right) \geq$ ${ }_{q}^{1} \widetilde{\mathcal{S}}(' \widetilde{\sigma})$, here D is from diagonal, which means that dropping the off-diagonal part of density matrix (this holds in any basis) results in entropy increasing. This has important implications, for instance, in gravity models emerging from (quantum) mechanical evolution theories. Pure diagonal con- 
figurations have higher entropy than the generic off-diagonal ones.

\section{Quantum generalizations of W- and thermodynamic entropy of mechanical systems}

QGIFs can characterized not only by a von Neumann entropy of type (60) but also by quantum analogs of entropy values used for classical geometric flows (associated thermodynamics entropy and W-entropy). Such values can be introduced and computed in explcity form using respective formulas (58), (59), (61) for classical conditional and mutual entropy used in formulas (51) and (52). The quantum formulas introduced in this section can be considered for geometric flows of arbitrary systems and not only for mechanical ones. So, we write $A, B, \ldots$ instead of $\widetilde{A}, \widetilde{B}, \ldots$ and define

$$
\begin{aligned}
{ }_{q} \widetilde{\mathcal{S}}_{A B} & =\operatorname{Tr}_{\mathcal{H}_{A B}}\left[\left({ }^{\prime} \widetilde{\sigma}_{A B}\right)\left({ }_{A B}{ }_{A B} \widetilde{\mathcal{S}}\right)\right] \text { and }{ }_{q}^{\prime} \widetilde{\mathcal{S}}_{A} \\
& =\operatorname{Tr}_{\mathcal{H}_{A}}\left[\left({ }^{\prime} \widetilde{\sigma}_{A}\right)\left({ }_{A}{ }_{A} \widetilde{\mathcal{S}}\right)\right],{ }_{q} \widetilde{\mathcal{S}}_{B} \\
& =\operatorname{Tr}_{\mathcal{H}_{B}}\left[\left({ }^{\prime} \widetilde{\sigma}_{B}\right)\left({ }_{B}{ }_{B} \widetilde{\mathcal{S}}\right)\right] .
\end{aligned}
$$

Similar formulas can be provided for the quantum version of W-entropy,

${ }_{q}^{1} \tilde{\mathcal{W}}_{A B}=\operatorname{Tr}_{\mathcal{H}_{A B}}\left[\left({ }^{\prime} \widetilde{\sigma}_{A B}\right)\left({ }_{A B}{ }_{A B} \tilde{\mathcal{W}}\right)\right]$ and

${ }_{q}^{1} \tilde{\mathcal{W}}_{A}=\operatorname{Tr}_{\mathcal{H}_{A}}\left[\left({ }^{\prime} \widetilde{\sigma}_{A}\right)\left({ }_{A}{ }_{A} \tilde{\mathcal{W}}\right)\right],{ }_{q}^{1} \tilde{\mathcal{W}}_{B}=\operatorname{Tr}_{\mathcal{H}_{B}}\left[\left({ }^{\prime} \widetilde{\sigma}_{B}\right)\left({ }_{B}^{1} \tilde{\mathcal{W}}\right)\right]$.

Such values describe QGIFs of Hamiltonian (quantum) mechanical systems.

The quantum probabilistic characteristics are described by the von Neumann entropy ${ }_{q} \widetilde{\mathcal{S}}\left({ }^{\prime} \widetilde{\sigma}_{A}\right)(60)$ and corresponding generalizations for $A B$ and $B$ systems

${ }_{q}^{1} \widetilde{\mathcal{S}}\left({ }^{\prime} \widetilde{\sigma}_{A B}\right):=\operatorname{Tr}{ }^{\prime} \widetilde{\sigma}_{A B} \log { }^{\prime} \widetilde{\sigma}_{A B}$ and

${ }_{q}^{1} \widetilde{\mathcal{S}}\left({ }^{\prime} \widetilde{\sigma}_{A}\right):=\operatorname{Tr}{ }^{\prime} \widetilde{\sigma}_{A} \log { }^{\prime} \widetilde{\sigma}_{A},{ }_{q}^{\prime} \widetilde{\mathcal{S}}\left({ }^{\prime} \widetilde{\sigma}_{B}\right):=\operatorname{Tr}{ }^{\prime} \widetilde{\sigma}_{B} \log { }^{\prime} \widetilde{\sigma}_{B}$.

Finally, we note that the entropies ${ }_{q} \widetilde{\mathcal{S}}_{A},{ }_{q} \widetilde{\mathcal{W}}_{A}$, and ${ }_{q} \widetilde{\mathcal{S}}\left({ }^{\prime} \widetilde{\sigma}_{A}\right)$ characterize respectively different thermodynamic, geometric flow and probabilistic properties of QGIFs of geometric mechanical Hamilton flows. In a similar form, we can omit the label " " " and derive respective formulas for quantum flows of Lagrange systems. Such a formalism is more sophisticate mathematically because the Lagrange generating functions can not be used directly for constructing base vectors for respective Hilbert spaces.

\section{Conditional and relative quantum entropy for QGIFs of mechanical systems}

For QGIFs, we can imitate formally many classical definitions for GIFs. As it is stated in section 3.4 of [38], the quantum versions are potentially misleading or not good or usual notions. This is not surprising in the case of geometric flows because they are characterized not only by certain probabilistic quantum entropies but also by G. Perelman W-entropy and geometric thermodynamic entropy. Let us outline the main equations for respective von Neumann and conditional and relative entropy of quantum mechanical geometric flows.

Using quantum matrix computations with formulas of type (58), (59), (61), we prove such quantum properties of entropies for QGIFs:

$$
\begin{aligned}
{ }_{q}^{\prime} \widetilde{\mathcal{S}}[A \mid B] & ={ }_{q}^{\prime} \widetilde{\mathcal{S}}_{A B}-{ }_{q}^{\prime} \widetilde{\mathcal{S}}_{B} \text { and }{ }_{q}^{\prime} \widetilde{\mathcal{J}}[A ; B] \\
& ={ }_{q}^{\prime} \widetilde{\mathcal{S}}_{A}+{ }_{q} \widetilde{\mathcal{S}}_{A B}+{ }_{q} \widetilde{\mathcal{S}}_{B} \geq 0 .
\end{aligned}
$$

Similar claims can be formulated (from small quantum perturbations, we can prove respective theorems) for the Neumann (60) and quantum W-entropy (29),

$$
\begin{aligned}
& { }_{q}^{\prime} \widetilde{\mathcal{S}}\left({ }^{\prime} \widetilde{\sigma}_{A \mid B}\right):={ }_{q}^{\prime} \widetilde{\mathcal{S}}\left({ }^{\prime} \widetilde{\sigma}_{A B}\right)-{ }_{q}^{\prime} \widetilde{\mathcal{S}}\left({ }^{\prime} \widetilde{\sigma}_{B}\right) \text { and }{ }_{q} \\
& \widetilde{\mathcal{J}}\left({ }^{\prime} \widetilde{\sigma}_{A ; B}\right):={ }_{q} \widetilde{\mathcal{S}}\left({ }^{\prime} \widetilde{\sigma}_{A}\right)-{ }_{q}^{\prime} \widetilde{\mathcal{S}}\left({ }^{\prime} \widetilde{\sigma}_{A B}\right)+{ }_{q}^{\prime} \widetilde{\mathcal{S}}\left({ }^{\prime} \widetilde{\sigma}_{B}\right) ; \\
& { }_{q}^{1} \tilde{\mathcal{W}}[A \mid B]={ }_{q}^{1} \widetilde{\mathcal{W}}_{A B}-{ }_{q}^{1} \widetilde{\mathcal{W}}_{B} \text { and }{ }_{q}^{1} \widetilde{\mathcal{J}}_{\widetilde{\mathcal{W}}}[A ; B] \\
& ={ }_{q}^{1} \tilde{\mathcal{W}}_{A}+{ }_{q}^{1} \widetilde{\mathcal{W}}_{A B}+{ }_{q}^{1} \widetilde{\mathcal{W}}_{B} \geq 0 \text {. }
\end{aligned}
$$

It should be noted that different entropies and related mutual information values characterize different properties of the QGIFs of mechanical Hamilton systems. The von Neumann type values ${ }_{q}^{\prime} \widetilde{\mathcal{S}}\left({ }^{\prime} \widetilde{\sigma}_{A \mid B}\right)$ and ${ }_{q}^{\prime} \widetilde{\mathcal{J}}\left({ }^{\prime} \widetilde{\sigma}_{A ; B}\right)$ can be used for proofs of entanglement and purifcation properties of such systems following standard methods of quantum information theory. Unlike the classical case, the quantum conditional entropy is not conditional on certain classical or quantum processes. But for QGIFs, the systems are with nonholonomic structure encoding classical and/or quantum mechanical systems. The conditional properties of such systems are encoded in ${ }_{q} \widetilde{\mathcal{S}}_{A}$ and ${ }_{q} \widetilde{\mathcal{J}}[A ; B]$, for thermodynamical models of QGIFs, and ${ }_{q}^{1} \widetilde{\mathcal{W}}_{A}$ and ${ }_{q}^{\prime} \widetilde{\mathcal{J}}_{\widetilde{\mathcal{W}}}[A ; B]$, for quantum geometric evolution flows.

\section{Monotonicity and monogamy of entanglement of relative entropy for QGIFs}

The relative entropies for QGIFs are positive just as for the classical GIFs. Using ${ }_{q} \widetilde{\mathcal{S}}\left({ }^{\prime} \widetilde{\sigma}_{A \mid B}\right)$, we can prove that such a quantum entropy is also monotonic (for proofs, we can use the same methods as in $[38,56]$, and posses also a strong subadditivity property as in [57]). The intuition behind the classical theory of probability is not applicable in a direct way for geometric flows and/or quantum systems. In this sense, the monotonicity of quantum relative entropies is a miracle.

Let us consider a very basic property of QGIFs described by the von Neumann entropy ${ }_{q} \widetilde{\mathcal{S}}\left({ }^{\prime} \widetilde{\sigma}_{A}\right)$. For a bipartite system $A B$ with two density matrices ' $\widetilde{\rho}_{A B}$ and ' $\widetilde{\sigma}_{A B}$, we can define the corresponding reduced density matrices on $A$, ' $\widetilde{\rho}_{A}=$ $\operatorname{Tr}_{B}\left({ }^{\prime} \widetilde{\rho}_{A B}\right)$ and ${ }^{\prime} \widetilde{\sigma}_{A}=\operatorname{Tr}_{B}\left({ }^{\prime} \widetilde{\sigma}_{A B}\right)$. The partial trace can only reduce the relative quantum entropy,

${ }_{q}^{1} \widetilde{\mathcal{S}}\left({ }^{\prime} \widetilde{\rho}_{A B} \|{ }^{\prime} \widetilde{\sigma}_{A B}\right) \geq{ }_{q} \widetilde{\mathcal{S}}\left({ }^{\prime} \widetilde{\rho}_{A} \|{ }^{\prime} \widetilde{\sigma}_{A}\right)$.

see also (34) and (55).

For a tripartite system $A B C$ with QGIF density matrix ${ }^{\prime} \widetilde{\rho}_{A B C}$ and above montonicity property, we can proved a 
strong subadditivity property for geometric flows of quantum mechanical Hamilton systems. There are used reduced density matrices and corresponding second density matrices

$$
\begin{aligned}
{ }^{\prime} \tilde{\rho}_{A} & =T_{B C}{ }^{\prime} \tilde{\rho}_{A B C},{ }^{\prime} \tilde{\rho}_{B C}=T_{A}{ }^{\prime} \tilde{\rho}_{A B C},{ }^{\prime} \tilde{\rho}_{A B}=T_{C}{ }^{\prime} \tilde{\rho}_{A B C} \text { and } \\
{ }^{\prime} \widetilde{\sigma}_{A B C} & ={ }^{\prime} \widetilde{\rho}_{A} \otimes{ }^{\prime} \widetilde{\rho}_{B C},{ }^{\prime} \tilde{\sigma}_{A B}=T_{C}{ }^{\prime} \widetilde{\sigma}_{A B C}={ }^{\prime} \widetilde{\rho}_{A} \otimes{ }^{\prime} \tilde{\rho}_{B} . \quad \text { (64) }
\end{aligned}
$$

Using above monotonicity property, we can write

$$
{ }_{q}^{\prime} \widetilde{\mathcal{S}}\left({ }^{\prime} \widetilde{\rho}_{A B C} \|{ }^{\prime} \widetilde{\sigma}_{A B C}\right) \geq{ }_{q}^{\prime} \widetilde{\mathcal{S}}\left({ }^{\prime} \widetilde{\rho}_{A B} \|{ }^{\prime} \widetilde{\sigma}_{A B}\right)
$$

and/or as the monotonicity of mutual information

$$
{ }_{q}^{\prime} \breve{\mathcal{J}}(A ; B C) \geq{ }_{q}^{\prime} \breve{\mathcal{J}}(A ; B),
$$

which is equivalent to the condition of strong subadditivity

$$
{ }_{q}^{1} \widetilde{\mathcal{S}}_{A B}+{ }_{q}^{\prime} \widetilde{\mathcal{S}}_{B C} \geq{ }_{q}^{1} \widetilde{\mathcal{S}}_{B}+{ }_{q}^{\prime} \widetilde{\mathcal{S}}_{A B C} .
$$

These formulas follow from (64); notations of type ${ }_{q}^{\prime} \widetilde{\mathcal{S}}\left({ }^{\prime} \widetilde{\sigma}_{A}\right)=$ ${ }_{q}^{\prime} \breve{\mathcal{S}}_{A},{ }_{q} \widetilde{\mathcal{S}}\left({ }^{\prime} \widetilde{\sigma}_{A B}\right)={ }_{q}^{\prime} \breve{\mathcal{S}}_{A B},{ }_{q}^{\prime} \widetilde{\mathcal{S}}\left({ }^{\prime} \widetilde{\sigma}_{A B C}\right)={ }_{q} \breve{\mathcal{S}}_{A B C}$; and definitions

$$
\begin{aligned}
& { }_{q}^{\prime} \widetilde{\mathcal{S}}\left({ }^{\prime} \widetilde{\rho}_{A B C} \| ' \widetilde{\sigma}_{A B C}\right)={ }_{q}^{\prime} \widetilde{\mathcal{S}}\left({ }^{\prime} \widetilde{\rho}_{A B C} \|{ }^{\prime} \widetilde{\rho}_{A} \otimes ' \widetilde{\rho}_{B C}\right) \\
& ={ }_{q} \breve{\mathcal{J}}(A ; B C):={ }_{q}^{1} \breve{\mathcal{S}}_{A}+{ }_{q} \breve{\mathcal{S}}_{B C}-{ }_{q} \breve{\mathcal{S}}_{A B C} ; \\
& { }_{q}^{\prime} \widetilde{\mathcal{S}}\left({ }^{\prime} \tilde{\rho}_{A B} \|{ }^{\prime} \widetilde{\sigma}_{A B}\right)={ }_{q}^{\prime} \widetilde{\mathcal{S}}\left({ }^{\prime} \tilde{\rho}_{A B} \|{ }^{\prime} \tilde{\rho}_{A} \otimes{ }^{\prime} \tilde{\rho}_{B}\right) \\
& =\left.\right|_{q} \breve{\mathcal{J}}(A ; B):={ }_{q}^{1} \breve{\mathcal{S}}_{A}+{ }_{q}^{\prime} \breve{\mathcal{S}}_{B}-{ }_{q}^{\prime} \breve{\mathcal{S}}_{A B} .
\end{aligned}
$$

The von Neumann entropy for QGIFs allows us to deduce an important property related to the monogamy of entanglement when a given qubit in a QGIF system $\widetilde{C}$ can be entangled with $\widetilde{D}$ (reducing ${ }_{q}^{\prime} \breve{\mathcal{S}}_{C D}$ ) or with $\widetilde{B}$ (reducing ${ }_{q}^{\breve{\mathcal{S}}_{B C}} \breve{C}_{\widetilde{B}}$ ), but not with both systems for set of 4 QGIF systems $\widetilde{A} \widetilde{B} \widetilde{C} \widetilde{D}$ (for mechanical systems, we can use tilde on symbols, which can be omitted for general GIFs). This follows from the possibility of purification of this type of entropy, which allows to find various equivalent systems. If we consider $A B C D$ in a pure state, then ${ }_{q}^{\prime} \breve{\mathcal{S}}_{A B}={ }_{q}^{1} \breve{\mathcal{S}}_{C D},{ }_{q}^{\prime} \widetilde{\mathcal{S}}_{A B C}={ }_{q}^{1} \breve{\mathcal{S}}_{D}$. The inequality (66) becomes ${ }_{q} \breve{\mathcal{S}}_{C D}+{ }_{q}^{\prime} \breve{\mathcal{S}}_{B C} \geq{ }_{q}^{1} \breve{\mathcal{S}}_{B}+{ }_{q}^{1} \breve{\mathcal{S}}_{D}$. We can consider, for instance, that ${ }_{q}^{\prime} \breve{\mathcal{S}}(C \mid D)={ }_{q} \breve{\mathcal{S}}_{C D}-{ }_{q} \breve{\mathcal{S}}_{D}<0$, or ${ }_{q} \breve{\mathcal{S}}(C \mid B)={ }_{q} \breve{\mathcal{S}}_{B C}-{ }_{q} \breve{\mathcal{S}}_{B}<0$, when the monogamy of entanglement follows from the non negative condition

$$
{ }_{q}^{1} \breve{\mathcal{S}}(C \mid D)+{ }_{q} \breve{\mathcal{S}}(C \mid B) \geq 0 .
$$

Above important conditions (65), (66) and (67) for the von Neumann entropy for QGIFs can be proven in a standard form for quantum information theory $[38,56,57]$. It is not clear if similar results can be proven for the thermodynamic entropy ${ }_{q} \widetilde{\mathcal{S}}_{A}$ or W-entropy ${ }_{q}^{1} \widetilde{\mathcal{W}}_{A}$. In principle, such values characterize certain complementary properties of QGIFs and relativistic quantum mechanical systems.

\subsubsection{Measurements for QGIFs and quantum channels}

In $\mathrm{QM}$, measurements involve projection onto orthogonal subspaces of a Hilbert space $\mathcal{H}_{A}$. The same formalism can be applied to QGIFs if we work with a density matrix ' $\widetilde{\sigma}$ of type (58) or (59).

\section{Generalized measurements for QGIFs of mechanical sys-} tems

Let us introduce a system of $\underline{s}=1, \ldots, \underline{k}$ orthogonal Hermitian projection operators $\pi_{\underline{s}}$ subjected to the conditions $\sum_{\underline{s}=1}^{\underline{k}} \pi_{\underline{s}}=1 ;\left(\pi_{\underline{s}}\right)^{2}=\pi_{\underline{s}}$; and $\pi_{\underline{s}} \pi_{\underline{s^{\prime}}}=0$ for $\underline{s} \neq \underline{s}^{\prime}$. Applying such a $\pi_{\underline{s}}$ to a pure quantum system $\mid \psi>\in \mathcal{H}$, we obtain an outcome $\underline{s}$ with probability $p_{\underline{s}}=\left\langle\psi\left|\pi_{\underline{s}}\right| \psi\right\rangle$, when the properties of $\pi_{\underline{s}}$ result in $\sum_{\underline{s}=1}^{\underline{k}} p_{\underline{s}}=1$. If a system $\widetilde{A}$ encodes a QGIF of a mechanical system characterized by a density matrix $' \widetilde{\sigma}$, the outcome $\underline{\underline{s}}$ is ' $\widetilde{p}_{\underline{\underline{s}}}=T r_{\mathcal{H}} \pi_{\underline{\underline{s}}} \mid \widetilde{\sigma}$. We endow such a probability ' $\widetilde{p}_{\underline{s}}$ with a typical label for a canonical Hamilton quantum system and respective geometric flows. A measurement with an outcome $\underline{s}$ changes the QGIFs and results in a new density matrix

$$
{ }^{\prime} \widetilde{\sigma}_{\underline{s}}=\pi_{\underline{s}} \text { ' } \widetilde{\sigma} \pi_{\underline{s}} /{ }^{\prime} \tilde{p}_{\underline{s}}
$$

encoding quantum information both from the geometric flows and the mechanical Hamilton structure.

In a more general context, measurements can be performed using an auxiliary system $C$. Such a system is not obligatory a mechanical one, of type $\widetilde{C}$ (it can be an electric device etc.). A procedure with auxiliary $C$ is called a "positive operator-valued measurement" or POVM) with Hilbert space $\mathcal{C}$. Conventionally, such a $\widetilde{C}$ is $k$-dimensional with a basis consisting from $\underline{k}$ vectors/states $\mid \underline{s}>\in \mathcal{C}$, for $\underline{s}=1,2, \ldots$, $k$. We can initialize such a $\mathcal{C}$-system in the state $\mid 1>$, then consider a combined system $\mathcal{C} \otimes \mathcal{H}$ and a corresponding unitary transform $U$ which, for instance, adjusts a time- and flow parameter - dependent Hamiltonian $H$ (if we a going to study quantum geometric flows of mechanical systems). The operator $U$ can be chosen that for any $\psi \in \mathcal{H}$, the result of such a transform is parameterized using arbitrary linear operators $E_{\underline{s}}$,

$U(\mid 1>\otimes \psi)=\sum_{\underline{s}=1}^{\underline{k}} \mid \underline{s}>\otimes E_{\underline{s}} \psi$ when $\sum_{\underline{s}=1}^{\underline{k}} E_{\underline{s}}^{\dagger} E_{\underline{s}}=1$

follows from the condition of unitarity (the symbol $\dagger$ is used for the Hermitian conjugation). We can label such values with "tilde" if they are considered for geometric mechanical flows, for instance, using $\widetilde{U}$ and $\widetilde{E}_{s}$. In princile, one can be used arbitrary operators, $U$ and $E_{\underline{s}}$, even the quantum density matrices, see below, will be taken for QGIFs. In general, projective measurements of the system $\mathcal{C} \otimes \mathcal{H}$ can be performed using the commuting projection operators 
$\pi_{\underline{s}}=|\underline{s}><\underline{s}| \otimes 1$ when the probability of outcome $\underline{s}$ is $p_{\underline{s}}=\left|E_{\underline{s}}\right| \psi>\left.\right|^{2}=<\psi E_{\underline{s}}^{\dagger} E_{\underline{s}} \mid \psi>$.

The described above POVM procedure can be applied for measurements of a QGIF system defined by a density matrix ' $\widetilde{\sigma}$, when the probability of outcome $\underline{s}$ is ' ' $\widetilde{p}_{\underline{s}}=$ $\operatorname{Tr}_{\mathcal{H}} E_{\underline{\underline{S}}}^{\dagger} E_{\underline{s}} ' \widetilde{\sigma}$. We can treat the numbers ' $\widetilde{p}_{\underline{\underline{s}}}$ as probabilities for any ' $\tilde{\sigma}$ because $E_{\underline{s}}^{\dagger} E_{\underline{s}} \geq 0$ for any $\underline{s}$ and (together with (69 )) this results in $\sum_{s=1}^{k}{ }^{\underline{k}} \widetilde{p}_{\underline{s}}=1$. It should be noted that $E_{\underline{s}}^{\dagger} E_{\underline{s}}$ are nonnegative Hermitian operators that add to 1 but not orthogonal projection ones. After a measurement with an outcome $\underline{s}$, the combined system $\mathcal{C} \otimes \mathcal{H}$ can be described by the density matrix for a "pure" quantum system. It can be parameterized in the form $\left(p_{\underline{s}}\right)^{-1}|\underline{s}><\underline{s}| \otimes E_{\underline{s}} \mid \psi><\psi E_{\underline{s}}^{\dagger}$, see (68), and, taking the partial trace over $\mathcal{C}$, we obtain a conventional density matrix $\left(p_{\underline{s}}\right)^{-1} E_{\underline{s}} \mid \psi><\psi E_{\underline{s}}^{\dagger}$ for the orginal system $\mathcal{H}$. QGIFs of such quantum mechanical systems can be described by mixed stated with density matrix ' $\widetilde{\sigma}$, when $\left(\widetilde{p}_{\underline{s}}\right)^{-1} E_{\underline{s}}$ ) $\widetilde{\sigma} E_{\underline{s}}^{\dagger}$ results in an outcome $\underline{s}$.

Finally, we note that above POVM constructions can be generalized for any Hilbert space of type $\mathcal{C} \otimes\left(\mathcal{H} \oplus \mathcal{H}^{\prime}\right)$ with linear transforms $E_{\underline{s}}: \mathcal{H} \rightarrow \mathcal{H}^{\prime}$, which is useful for elaborating on generalized quantum models and information theory.

\section{Quantum channels for QGIFs}

For modeling quantum information flow theories, a corresponding density matrix evolves both in a QM form and as a geometric flow evolution process. The usual Hamiltonian evolution of a state $|\psi>\rightarrow U| \psi>$ can be described by a unitary operator $U$ a Hamiltonian $\widehat{H}$ corresponding to a canonical relativistic Hamiltonian $\widetilde{H}$ (and respective Hessian ${ }^{\prime} \widetilde{g}^{a b}(x, p)(4)$ and canonical d-metric ' $\left.\widetilde{\mathbf{g}}(15)\right)$ or by a ther-

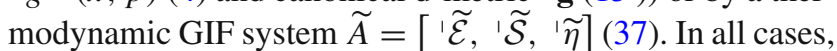
we can introduce the von Neumann entropy ${ }_{q}^{\prime} \widetilde{\mathcal{S}}\left({ }^{\prime} \widetilde{\sigma}_{A}\right)(60)$, and conditional entropy ${ }_{q}^{\prime} \widetilde{\mathcal{S}}[\widetilde{A} \mid \widetilde{B}](62)$, which are invariant under unitary transforms ' $\widetilde{\sigma}_{A} \in U{ }^{\prime} \widetilde{\sigma}_{A} U^{-1}$. Such QGIFs are also characterized by W-entropy ${ }_{q}^{1} \widetilde{\mathcal{W}}_{A}(29)$ and or ${ }_{q}^{1} \widetilde{\mathcal{S}}_{A}(37)$.

Let us analyze how the notion of quantum channels can be elaborated for QGIFs of mechanical Hamilton systems. We consider again an extended system $\mathcal{C} \otimes \mathcal{H}$ enabled with a density matrix $' \breve{\sigma}=|1><1| ' \widetilde{\sigma}$, where ' $\widetilde{\sigma}$ is a density matrix on $\mathcal{H}$. Unitary maps ' $\breve{\sigma} \rightarrow ' \breve{\sigma}^{\prime}$ ', and with a trace induced matrix ' $\tilde{\sigma}^{\prime}$ on $\mathcal{H}$, can be parameterized in the form (69),

$$
\begin{aligned}
' \breve{\sigma}^{\prime} & =U ' \breve{\sigma} U^{-1}=\sum_{\underline{s}, \underline{s^{\prime}}=1}^{\underline{k}}\left|\underline{s}><\underline{s^{\prime}}\right| \otimes E_{\underline{s}}{ }^{\prime} \tilde{\sigma} E_{\underline{s}}^{\dagger} \text { and ' } \sigma^{\prime} \\
& =\operatorname{Tr}_{\mathcal{C}}{ }^{\prime} \breve{\sigma}^{\prime}=\sum_{\underline{s}=1}^{\underline{k}} E_{\underline{s}}{ }^{\prime} \widetilde{\sigma} E_{\underline{s}}^{\dagger} .
\end{aligned}
$$

In result, we can define certain "quantum channels" for evolution of QGIF density matrices for mechanical systems as operations ' $\widetilde{\sigma} \rightarrow \sum_{\underline{s}=1}^{\underline{k}} E_{\underline{s}}{ }^{\prime} \widetilde{\sigma} E_{\underline{s}}^{\dagger}$, where the so-called Kraus operators $E_{\underline{s}}$ are subjected to the condition $\sum_{\underline{s}=1}^{\underline{k}} E_{\underline{s}} E_{\underline{s}}^{\dagger}=1$. If we consider only one Kraus operator, we obtain as a special case the unitary evolution of a QGIF system.

We can consider quantum channels for the relative entropy and respective inequality conditions (63) which are written in the form

${ }_{q}^{\prime} \widetilde{\mathcal{S}}\left({ }^{\prime} \widetilde{\rho} \| \mid \widetilde{\sigma}\right) \geq{ }_{q}^{\prime} \widetilde{\mathcal{S}}(\mid \widetilde{\rho} \| ' \widetilde{\sigma})$

for ${ }^{\prime} \tilde{\rho} \rightarrow \sum_{\underline{s}=1}^{\underline{k}} E_{\underline{s}}{ }^{\prime} \tilde{\rho}_{\underline{s}}^{\dagger}$ and ${ }^{\prime} \tilde{\sigma} \rightarrow \sum_{\underline{s}=1}^{k} E_{\underline{s}}{ }^{\prime} \tilde{\sigma} E_{\underline{s}}^{\dagger}$, when the fist step of initialization consists in replacing ' $\widetilde{\rho}$ and ' $\widetilde{\sigma}$, respectively, by $\mid 1><1 \otimes ' \widetilde{\rho}$ and $\mid 1><1 \otimes ' \widetilde{\sigma}$. This is a very general statement on monotonicity of relative entropy and the von Neumann entropy for QGIFs of mechanical systems. The properties of Kraus operators for quantum channels are similar to those outlined in paragraphs (1)-(6) in section 3.7 of [38], see also references therein. There are two differences: the first one is that we consider geometric flow evolution of density matrices and that such rich quantum and geometric flow evolutions are characterized by additional inequalities for the quantum versions of thermodynamic entropy and W-entropy.

\section{Thermodynamics of QGIFs and quantum channels}

Let us consider a thermal quantum density matrix as in QM, ${ }_{q}^{1} \widetilde{\sigma}={ }_{q}^{1} \widetilde{\mathcal{Z}}^{-1} e^{-\beta \widetilde{H}}$, with $\beta=1 / T, \tau=T$. We define for the conditional quantum entropy for geometric flows of Hamilton mechanical systems

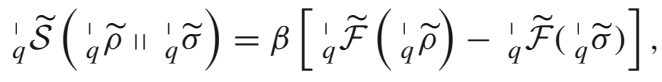

where the free energy corresponding to a second density matrix ${ }_{q}^{1} \widetilde{\rho}$ is ${ }_{q}^{1} \widetilde{\mathcal{F}}\left({ }_{q}^{1} \widetilde{\rho}\right):={ }_{q}^{1} \widetilde{\mathcal{E}}\left({ }_{q}^{1} \widetilde{\rho}\right)-\left.T\right|_{q} ^{\prime} \widetilde{\mathcal{S}}\left({ }_{q}^{1} \widetilde{\rho}\right)$. The energy operator is defined and computed as ${ }_{q}^{1} \widetilde{\mathcal{E}}\left({ }_{q}^{1} \widetilde{\rho}\right)=\operatorname{Tr}\left[\left({ }_{q}^{1} \widetilde{\rho}\right) \widetilde{H}\right]$ and the thermodynamic entropy is

${ }_{q}^{\prime} \widetilde{\mathcal{S}}\left({ }_{q}^{1} \widetilde{\rho}\right):=\beta_{q}^{\prime} \widetilde{\mathcal{E}}\left({ }_{q}^{1} \widetilde{\rho}\right)+\log _{q}{ }_{q} \widetilde{\mathcal{Z}}\left({ }_{q}^{1} \widetilde{\rho}\right)$.

If $\log { }_{q}^{\prime} \widetilde{\mathcal{Z}}$ is independent on ${ }_{q}^{1} \tilde{\rho}$, we obtain ${ }_{a} \widetilde{\mathcal{S}}\left({ }_{q}^{\prime} \widetilde{\sigma} \|{ }_{q}^{\prime} \widetilde{\sigma}\right)=$ 0 . For any quantum channel preserving the thermal equilibrium at temperature $T$, there is a map ${ }_{q}^{i} \tilde{\sigma}$ to itself and transforms ${ }_{q}^{\prime} \widetilde{\rho}$ to a general density matrix ${ }_{q}^{1} \widetilde{\rho}^{\prime}$. In such a quantum channel the entropy decreases following formulas

$$
\begin{aligned}
& { }_{q}^{1} \widetilde{\mathcal{S}}\left({ }_{q}^{\prime} \tilde{\rho} \|_{\|}{ }_{q}^{\prime} \widetilde{\sigma}\right) \geq{ }_{q}^{\prime} \widetilde{\mathcal{S}}\left({ }_{q}^{1} \widetilde{\rho}^{\prime} \|{ }_{q}^{\prime} \widetilde{\sigma}\right) \text { and } \\
& { }_{q}^{1} \tilde{\mathcal{F}}\left({ }_{q}^{1} \widetilde{\rho}\right) \geq{ }_{q}{ }_{q} \widetilde{\mathcal{F}}\left({ }_{q}{ }_{q} \widetilde{\rho}^{\prime}\right) \text {. }
\end{aligned}
$$

For quasi-classical approximations, we consider that such formulas transform into similar ones, see (55), for the state densities of type ' $\widetilde{\sigma}(54)$. 


\section{Outlook and conclusions}

In this paper, we put emphasis on the roles of entropic values derived from Perelman-Lyapunov type functionals $[1,36]$ in elaborating relativistic models of geometric flow evolution of Lagrange-Hamilton mechanical systems and possible applications in classical and quantum information theory. Our aim was to seek answer to wether the incorporation of fundamental geometric objects in relativistic mechanics into canonical noholonomic structures on (co) tangent Lorentz bundles allow a new (J. Kern type) geometrization in terms of certain generalized (pseudo) Riemannian and FinslerLagrange-Hamilton spaces $[34,35,43,45]$. Due to Grigory Perelman, such geometric constructions can be characterized by $\mathrm{W}$-entropy functionals and respective statistical/ geometric thermodynamic functionals like average flow energy, flow entropy and flow fluctuation, see further developments and applications in physics [19-22,25,26,50,53].

Here it should be emphasized that such concepts of "nonarea, non-holographic, non-conformal ..." entropy are more general that those based on the Bekenstein-Hawking thermodynamics [39-42]. In our approach, the fundamental geometric and physical objects are defined by analogous metrics, nonlinear and linear connections, and their curvatures, canonically determined by Hessians of respective Lagrange and/or Hamilton generating functions. Corresponding entropic and thermodynamic type values can be computed for various classes of exact and parametric solutions (not only black hole type ones) in geometric flow evolution and (modified) gravity theories.

The work presented here indicates that G. Perelman's ideas and geometric methods with W-entropy and associated thermodynamic models for Ricci flows presented not only an important tool for proving the Poincaré-Thurston hypothesis. The constructions can be generalized for various types of relativistic and/or non-Riemannian geometries which allow to elaborate on further developments for noncommutative, supersymmetric, stochastic and quantum geometries [23,24, $27,54,55]$. Although in this paper we investigated only flows of geometric mechanical Lagrange-Hamilton models elaborated on (co) tangent Lorentz bundles, and did the hole analysis based on classical and quantum mechanical Hamilton structures, our study sheds light on the importance of such constructions in elaborating new directions in quantum information theory $[37,38,47-49]$. We note that the conjecture that gravity can be thought of as an entropic force [51,52] can be proven for certain classes of nonholonomic deformations of G. Perelman's functionals [27-29]. Using the results of this and partner works [27-29], we conclude that such proofs can be performed for the emergent gravity from classical and quantum mechanical Lagrange-Hamilton theories.

The results of Sect. 4 support also the conclusion that using advanced geometric methods we can elaborate on basic ingredients of the geometric flow information, QGIF, theory. We close with the remark that in our future works there will be considered some more special topics of QGIFs such as teleportation and conditional geometric flow entropy; relative entropy and hypothesis geometric flow testing; how to encode classical geometric flow information in quantum states; geometric classical and quantum flow entanglement and emergent gravity theories.

Acknowledgements This research develops the author's former programs partially supported by IDEI, PN-II-ID-PCE-2011-3-0256, CERN and DAAD and contains certain results for new grant proposals. The UAIC co-affiliation refers to the Project IDEI hosted by that University during 2012-2015, when the bulk of main ideas and results of this and partner works were elaborated. The third co-affiliation reflects a present visiting position at Yu. Fedkovych Chernivtsi National University. The author is grateful to D. Singleton, P. Stavrinos, M. V. Tkach, and Ju. O. Seti for collaboration and supporting his research on geometric and quantum methods in physics and information theory.

Data Availability Statement This manuscript has no associated data or the data will not be deposited. [Authors' comment: This is a theoretical study and no experimental data has been listed.]

Open Access This article is licensed under a Creative Commons Attribution 4.0 International License, which permits use, sharing, adaptation, distribution and reproduction in any medium or format, as long as you give appropriate credit to the original author(s) and the source, provide a link to the Creative Commons licence, and indicate if changes were made. The images or other third party material in this article are included in the article's Creative Commons licence, unless indicated otherwise in a credit line to the material. If material is not included in the article's Creative Commons licence and your intended use is not permitted by statutory regulation or exceeds the permitted use, you will need to obtain permission directly from the copyright holder. To view a copy of this licence, visit http://creativecomm ons.org/licenses/by/4.0/.

Funded by $\mathrm{SCOAP}^{3}$.

\section{References}

1. G. Perelman, The entropy formula for the Ricci flow and its geometric applications. arXiv:math.DG/0211159

2. G. Perelman, Ricci flow with surgery on three-manifolds. arXiv:math.DG/0303109

3. G. Perelman, Finite extintion time for the solutions to the Ricci flow on certain three-manifolds. arXiv:math.DG/0307245

4. W. Thurston, Three-dimensional geometry and topology, Vol. 1. Edited by S. Levy, Princeton Mathematical Series, vol. 35 (Princeton University Press, Princeton, 1997)

5. W. Thurston, The Geometry and Topology of Three-Manifolds, Princeton lectures notes on geometric structures on 3-manifolds (1980), see updated electronic version following a MSRI. http:// library.msri.org/books/gt $3 \mathrm{~m} /$

6. W.P. Thurston, Three-dimensional manifolds, Kleinian groups and hyperbolic geometry, American Mathematical Society. Bull. New Ser. 6(3), 357-381 (1982)

7. D. Friedan, Nonlinear models in $2+\varepsilon$ dimensions, Ph.D. Thesis (Berkely) LBL-11517, UMI-81-13038, Aug, p. 212 (1980)

8. D. Friedan, Nonlinear models in $2+\varepsilon$ dimensions. Phys. Rev. Lett. 45, 1057-1060 (1980) 
9. D. Friedan, Nonlinear models in $2+\varepsilon$ dimensions. Ann. Phys. 163, 318-419 (1985)

10. R.S. Hamilton, Three-manifolds with postive Ricci curvature. J. Differ. Geom. 17, 255-306 (1982)

11. R.S. Hamilton, The Ricci flow on surfaces. In Mathematics and General Relativity, Contemp. Math., vol. 71, (American Mathematical Society, Providence, 1988), pp. 237-262

12. R.S. Hamilton, Surveys in Differential Geometry, vol. 2 (International Press, Boston, 1995), pp. 7-136

13. H.-D. Cao, H.-P. Zhu, A complete proof of the Poincaré and geometrization conjectures - application of the Hamilton-Perelman theory of the Ricci flow. Asian J. Math. 10, 165-495 (2006)

14. J.W. Morgan, G. Tian, Ricci flow and the Poincaré conjecture, AMS. Clay Math. Monogr. 3 (2007)

15. B. Kleiner, J. Lott, Notes on Perelman's papers. Geom. Topol. 12, 2587-2855 (2008)

16. R. Abraham, J.E. Marsden, Foundations of Mechanics (BenjaminCummings, London, 1978)

17. V.I. Arnol'd, Mathematical Methods of Classical Mechanics (Springer, New York, 1989)

18. M. de Leon, P. Rodrigues, Generalized Classical Mechanics and Field Theory (North-Holland, 1985)

19. S. Vacaru, Nonholonomic Ricci flows: II. Evolution equations and dynamics. J. Math. Phys. 49, 043504 (2008). arXiv:math.DG/0702598

20. S. Vacaru, The entropy of Lagrange-Finsler spaces and Ricci flows. Rep. Math. Phys. 63, 95-110 (2009). arXiv:math.DG/0701621

21. L. Bubuianu, S. Vacaru, Dynamical equations and Lagrange-Ricci flow evolution on prolongation lie algebroids. Can. J. Phys. 97, 133-144 (2019). arXiv:1108.4333v3

22. S. Vacaru, Almost Kaehler Ricci flows and Einstein and LagrangeFinsler structures on lie algebroids. Medit. J. Math. 12, 1397-1427 (2015). arXiv:1306.2813 [math.DG]

23. S. Vacaru, Spectral functionals, nonholonomic Dirac operators, and noncommutative Ricci flows. J. Math. Phys. 50, 073503 (2009). arXiv:0806.3814

24. S. Rajpoot, S. Vacaru, On supersymmetric geometric flows and R2 inflation from scale invariant supergravity. Ann. Phys. 384, 20-60 (2017). arXiv: 1606.06884

25. V. Ruchin, O. Vacaru, S. Vacaru, Perelman's W-entropy and statistical and relativistic thermodynamic description of gravitational fields. Eur. Phys. J. C 77, 184 (2017). arXiv: 1312.2580

26. T. Gheorghiu, V. Ruchin, O. Vacaru, S. Vacaru, Geometric flows and Perelmans thermodynamics for black ellipsoids in R2 and Einstein gravity theories. Ann. Phys. 369, 1-35 (2016). arXiv: 1602.08512

27. L. Bubuianu, S. Vacaru, Black holes with MDRs and BekensteinHawking and Perelman entropies for Finsler-Lagrange-Hamilton spaces. Ann. Phys. 404, 10-38 (2019). arXiv:1812.02590

28. S. Vacaru, L. Bubuianu, Classical and quantum geometric information flows and entanglement of relativistic mechanical systems. Quantum Inf. Process QINP 18, 376 (2019). arXiv:1905.13015

29. S. Vacaru, E. Veliev, L. Bubuianu, Exact solutions in emergent gravity theories and generalized G. Perelman entropy for geometric flows; version 3 will modify former 2 versions of arXiv: 1904.05149

30. S. Capozziello, V. Faraoni, Beyond Einstein Gravity (Springer, Berlin, 2010)

31. S. Basilakos, A.P. Kouretsis, E.N. Saridakis, P. Stavrinos, Resembling dark energy and modified gravity with Finsler-Randers cosmology. Phys. Rev. D 83, 123510 (2013)

32. T. Elghozi, N.E. Mavromatos, M. Sakellariadou, M.F. Yusaf, The D-meterial univese. JCAP 1602(02), 60 (2016)

33. S. Nojiri, S.D. Odintsov, V.K. Oikonomou, Modified gravity theories in nutshell: inflation, bounce and late-time evolution. Phys. Rept. 692, 1-104 (2017). arXiv:1705.11098
34. S. Vacaru, On axiomatic formulation of gravity and matter field theories with MDRs and Finsler-Lagrange-Hamilton geometry on (co) tangent Lorentz bundles. arXiv:1801.06444

35. L. Bubuianu, S. Vacaru, Axiomatic formulations of modified gravity theories with nonlinear dispersion relations and FinslerLagrange-Hamilton geometry. Eur. Phys. J. C 78, 969 (2018)

36. A.M. Lyapunov, The General Problem of the Stability of Motion (In Russian), Doctoral dissertation (Univ. Kharkov, 1892) [English translations: 1) Stability of Motion (Academic Press, New-York \& London 1966); 2) The General Problem of the Stability of Motion, A. T. Fuller translation (Taylor \& Francis, London 1992), being included a biography and an extensive bibliography of Lyapunov's works]

37. J. Preskill, Lecture notes. http://www.theory.caltech.edu/ preskill/ ph219/index.html\#lecture

38. E. Witten, A mini-introduction to information theory. arXiv: 1805.11965

39. J.D. Bekenstein, Black holes and the second law. Nuovo Cim. Lett. 4, 737-740 (1972)

40. J.D. Bekenstein, Black holes and entropy. Phys. Rev. D 7, 2333 2346 (1973)

41. J.M. Bardeen, B. Carter, S.W. Hawking, The four laws of black hole mechanics. Commun. Math. Phys. 31, 161 (1973)

42. S.W. Hawking, Particle creation by black holes. Commun. Math. Phys. 43, 199-220 (1975)

43. J. Kern, Lagrange Geometry. Arch. der Math. (Basel) 25, 438-443 (1974)

44. M. Matsumoto, Connections, metrics and almost complex structures on tangent bundles. J. Math. Kyoto Univ. 5, 251-278 (1966)

45. M. Matsumoto, Foundations of Finsler Geometry and Special Finsler Spaces (Kaseisha Press, Tokio, 1986)

46. C.W. Misner, K.S. Thorne, J.A. Wheeler, Gravitation (Freeman, New York, 1973)

47. M.A. Nielsen, I.L. Chuang, Quantum Computation and Quantum Information (Cambridge University Press, Cambridge, 2000)

48. T.M. Cover, J.A. Thomas, Elements of Information Theory (Wiley, New York, 1991)

49. M.M. Wilde, Quantum Information Theory (Cambridge University Press, Cambridge, 2013)

50. M. Alexiou, P. Stavrinos, S. Vacaru, Nonholonomic Ricci flows of Riemann metrics and Lagrange-Finsler geometry, J. Phys. Math. 7(2), 14 (2016). arXiv:math.DG/0612162 [a paper modifying the version put in]

51. E.P. Verlinde, On the origin of gravity and the laws of Newton. JHEP 1104, 029 (2011). arXiv: 1001.0785

52. E.P. Verlinde, Emergent gravity and the dark universe. SciPost Phys. 2(3), 016 (2017). arXiv:1611.02269

53. S. Vacaru, Locally anisotropic kinetic processes and thermodynamics in curved spaces. Ann. Phys. (N. Y.) 290, 83-123 (2001). arXiv:gr-qc/0001060

54. S. Vacaru, Nonholonomic relativistic diffusion and exact solutions for stochastic Einstein spaces. Eur. Phys. J. Plus 127, 32 (2012). arXiv: 1010.0647

55. S. Vacaru, Diffusion and self-organized criticality in Ricci flow evolution of Einstein and Finsler spaces. SYMMETRY Cult. Scie. 23(2), 105-124 (2013). arXiv:1010.2021 [ISSN 0865-4824 (printed), ISSN 2226-1877 (online), Thematic Issue: Field theories on Finsler Space (Symmetries with Finsler metric, 2013)]

56. E.H. Lieb, M.B. Ruskai, Proof of the strong subadditivity of quantum mechanical entropy. J. Math. Phys. 14, 1938-1941 (1973)

57. E. Witten, Notes on some entanglement properties of quantum field theory. Rev. Mod. Phys. 90, 45003 (2018). arXiv:1803.04993 